NASA/CR—2008-215480

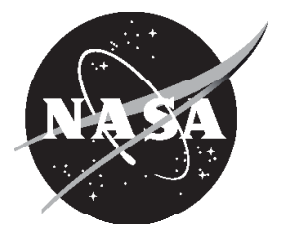

Effect of Adding a Regenerator to Kornhauser's MIT "Two-Space" (Gas-Spring+Heat Exchanger) Test Rig

Asuquo B. Ebiana and Praveen Gidugu

Cleveland State University, Cleveland, Ohio 


\section{NASA STI Program . . . in Profile}

Since its founding, NASA has been dedicated to the advancement of aeronautics and space science. The NASA Scientific and Technical Information (STI) program plays a key part in helping NASA maintain this important role.

The NASA STI Program operates under the auspices of the Agency Chief Information Officer. It collects, organizes, provides for archiving, and disseminates NASA's STI. The NASA STI program provides access to the NASA Aeronautics and Space Database and its public interface, the NASA Technical Reports Server, thus providing one of the largest collections of aeronautical and space science STI in the world. Results are published in both non-NASA channels and by NASA in the NASA STI Report Series, which includes the following report types:

- TECHNICAL PUBLICATION. Reports of completed research or a major significant phase of research that present the results of NASA programs and include extensive data or theoretical analysis. Includes compilations of significant scientific and technical data and information deemed to be of continuing reference value. NASA counterpart of peer-reviewed formal professional papers but has less stringent limitations on manuscript length and extent of graphic presentations.

- TECHNICAL MEMORANDUM. Scientific and technical findings that are preliminary or of specialized interest, e.g., quick release reports, working papers, and bibliographies that contain minimal annotation. Does not contain extensive analysis.

- CONTRACTOR REPORT. Scientific and technical findings by NASA-sponsored contractors and grantees.

- CONFERENCE PUBLICATION. Collected papers from scientific and technical conferences, symposia, seminars, or other meetings sponsored or cosponsored by NASA.

- SPECIAL PUBLICATION. Scientific, technical, or historical information from NASA programs, projects, and missions, often concerned with subjects having substantial public interest.

- TECHNICAL TRANSLATION. Englishlanguage translations of foreign scientific and technical material pertinent to NASA's mission.

Specialized services also include creating custom thesauri, building customized databases, organizing and publishing research results.

For more information about the NASA STI program, see the following:

- Access the NASA STI program home page at http://www.sti.nasa.gov

- E-mail your question via the Internet to help@ sti.nasa.gov

- Fax your question to the NASA STI Help Desk at 301-621-0134

- Telephone the NASA STI Help Desk at 301-621-0390

- Write to: NASA Center for AeroSpace Information (CASI) 7115 Standard Drive Hanover, MD 21076-1320 


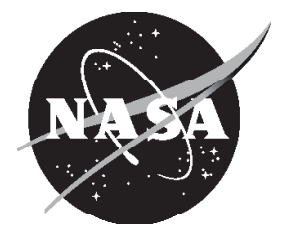

\section{Effect of Adding a Regenerator to Kornhauser's MIT "Two-Space" (Gas-Spring+Heat Exchanger) Test Rig}

Asuquo B. Ebiana and Praveen Gidugu

Cleveland State University, Cleveland, Ohio

Prepared for the

Sixth International Energy Conversion Engineering Conference (IECEC)

sponsored by the American Institute of Aeronautics and Astronautics

Cleveland, Ohio, July 28-30, 2008

Prepared under Grant NNC-05AA24A

National Aeronautics and

Space Administration

Glenn Research Center

Cleveland, Ohio 44135 


\section{Acknowledgments}

The work described in this paper was performed for the Science Mission Directorate (SMD) and the Radioisotope Power System (RPS) Development Program which provided the National Aeronautics and Space Administration (NASA) funding for this grant. We are grateful for the sponsorship of this contract by the NASA Glenn Research Center (GRC) under grant number

NNC-05AA24A. We wish to acknowledge the valuable and insightful guidance we received from our contract monitor, Dr. Roy Tew Jr., and the generous disposition of his time throughout the duration of this study.

Level of Review: This material has been technically reviewed by NASA technical management.

Available from

NASA Center for Aerospace Information 7115 Standard Drive

Hanover, MD 21076-1320
National Technical Information Service 5285 Port Royal Road Springfield, VA 22161

Available electronically at http://gltrs.grc.nasa.gov 


\title{
Effect of Adding a Regenerator to Kornhauser's MIT "Two-Space" (Gas-Spring+Heat Exchanger) Test Rig
}

\author{
Asuquo B. Ebiana and Praveen Gidugu \\ Cleveland State University \\ Cleveland, Ohio 44115
}

\begin{abstract}
This study employed entropy-based second law post-processing analysis to characterize the various thermodynamic losses inside a 3-space solution domain (gas spring + heat exchanger + regenerator) operating under conditions of oscillating pressure and oscillating flow. The 3-space solution domain is adapted from the 2-space solution domain (gas spring + heat exchanger) in Kornhauser's MIT test rig by modifying the heat exchanger space to include a porous regenerator system. A thermal non-equilibrium model which assumes that the regenerator porous matrix and gas average temperatures can differ by several degrees at a given axial location and time during the cycle is employed. An important and primary objective of this study is the development and application of a thermodynamic loss post-processor to characterize the major thermodynamic losses inside the 3-space model. It is anticipated that the experience gained from thermodynamic loss analysis of the simple 3-space model can be extrapolated to more complex systems like the Stirling engine. It is hoped that successful development of loss post-processors will facilitate the improvement of the optimization capability of Stirling engine analysis codes through better understanding of the heat transfer and power losses. It is also anticipated that the incorporation of a successful thermal nonequilibrium model of the regenerator in Stirling engine CFD analysis codes, will improve our ability to accurately model Stirling regenerators relative to current multi-dimensional thermal-equilibrium porousmedia models.
\end{abstract}

\begin{tabular}{|c|c|}
\hline \multicolumn{2}{|c|}{ Latin letters } \\
\hline $\mathrm{AE}$ & Available energy \\
\hline AEfric & $\mathrm{AE}$ (friction) \\
\hline $\mathrm{AEQ}_{\mathrm{w}}$ & $\mathrm{AE}$ (surface heat flow) \\
\hline $\mathrm{AEQ}_{\mathrm{x}}$ & AE(axial heat flow) \\
\hline AEDiscr & Discrepancy (AE int vs. $\left.\mathrm{AE}_{\text {ext }}\right)$ \\
\hline $\mathrm{c}$ & Specific heat \\
\hline $\mathrm{c}_{1}, \mathrm{c}_{2}$ & constants \\
\hline $\mathrm{D} / \mathrm{Dt}$ & Total derivative \\
\hline ext & External \\
\hline $\mathrm{h}$ & Enthalpy \\
\hline$\widetilde{\mathrm{I}}$ & Identity vector \\
\hline I & internal \\
\hline k & Thermal conductivity \\
\hline $\mathrm{N}_{\mathrm{k}}$ & Axial conductivity enhancement \\
\hline $\mathrm{n}_{\alpha}$ & Unit normal vector \\
\hline $\mathrm{p}$ & Pressure \\
\hline $\operatorname{Pr}$ & Prandtl number \\
\hline pe & Potential energy \\
\hline Q & Heat transfer \\
\hline $\overrightarrow{\mathrm{q}}$ & Heat flux vector \\
\hline$r_{\mathrm{v}}$ & Volume ratio \\
\hline S & Piston stroke \\
\hline s & Mass specific entropy \\
\hline$s^{\prime \prime \prime}$ & Volumetric entropy gen. rate \\
\hline $\mathrm{T}$ & Temperature \\
\hline \#tspc & Number of time steps per cycle \\
\hline $\mathrm{t}$ & Time \\
\hline$\Delta \mathrm{t}$ & Time step \\
\hline $\mathrm{u}$ & Internal energy \\
\hline
\end{tabular}

$\begin{array}{ll} & \text { Nomenclature } \\ \hat{\mathrm{u}} & \text { Specific internal energy } \\ \overrightarrow{\mathrm{u}} & \text { Velocity vector } \\ \mathrm{V} & \text { Volume, Averaging volume } \\ \mathrm{H} & \text { Convective heat transfer coeff. } \\ \mathrm{v} & \text { Mass specific volume } \\ \hat{\lambda} & \text { Spatial deviation quantity } \\ \langle\rangle & \text { Volume average quantity } \\ \mathrm{W} & \text { Work } \\ \mathrm{x} & \text { Position vector } \\ \mathrm{x}, \mathrm{y}, \mathrm{z} & \text { Cartesian coordinates } \\ \mathrm{x}_{\mathrm{i}} & \text { Mass fraction } \\ \boldsymbol{G r e e k} & \text { letters } \\ \alpha_{v} & \text { Surface area per unit volume } \\ \boldsymbol{\varepsilon} & \text { Volume fraction or porosity } \\ \nabla & \text { Gradient } \\ \mu & \text { Viscous coefficient } \\ \Phi & \text { Dissipation function } \\ \rho & \text { Density } \\ \theta & \text { Crank angle } \\ \vec{\Omega} & \text { Vorticity vector } \\ \boldsymbol{S u b s c r i p t s} \\ \alpha & \text { Fluid phase } \\ \beta & \text { Solid phase, weight factor } \\ \text { ext } & \text { External } \\ \text { cond } & \text { Conductive } \\ \text { gen } & \text { Generation } \\ \mathrm{i} & \text { inner face of the piston } \\ & \end{array}$

Nomenclature

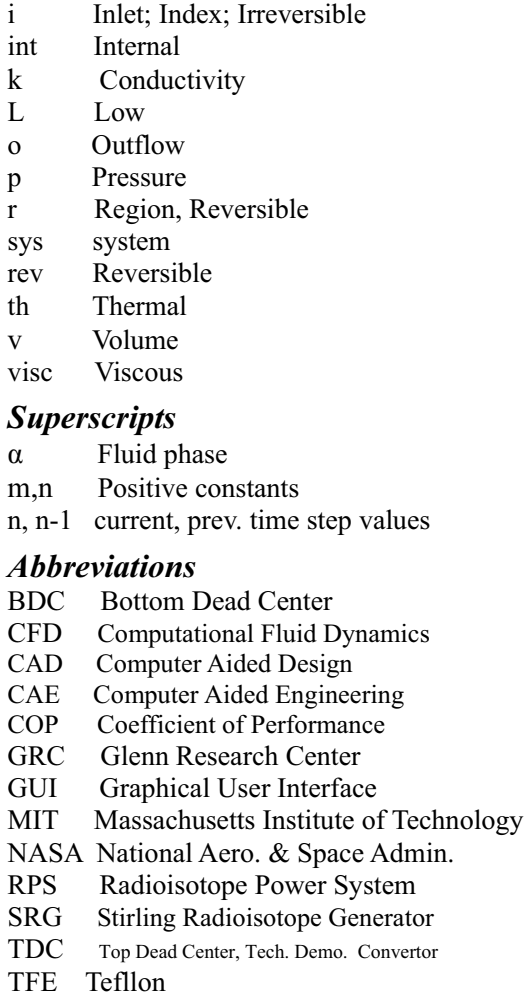




\section{Introduction}

\section{A. Background}

Under the auspices of NASA's Radioisotope Power System (RPS) development program, multiple efforts are currently underway, both in-house at NASA Glenn Research Center (GRC) and under various grants and contracts, to develop a high-efficiency Stirling Radioisotope Generator (SRG) for possible use on future NASA Space Science Missions. The SRG is being developed for multi-mission use including providing electric power for unmanned Mars rovers and deep space missions in environments with and without atmospheres. One of the multiple efforts underway includes the development of a multi-dimensional Stirling computational fluid dynamics code, including second law analysis post-processing to separate various thermodynamic losses ${ }^{34,37}$ in Stirling engines. The second law loss analysis effort documented in this paper supports the efforts underway in the Stirling research community and is sponsored by NASA grant NNC05AA24A.

Heat engines convert heat to useful work. This conversion process is inherently irreversible due to the non-ideal nature of power systems. Internal system irreversibilities resulting from viscous friction, heat transfer and nonequilibrium processes destroy part of the available work of a power system resulting in thermodynamic losses which impact engine thermodynamic performance adversely. There are two major design objectives for space-power applications of reciprocating heat engines like the Stirling engine: (1) To maximize performance by minimizing thermodynamic losses - in order to minimize fuel requirements (expensive radioisotopes for example), and (2) To minimize system mass - in order to minimize propulsion fuel requirements. In order to improve the engine thermodynamic performance, it is necessary to identify and minimize the features guilty of the available energy loss within the system. Thermo-fluid system loss analysis and management is clearly an area of study that is generating a great deal of interest.

Optimal engine performance requires good heat transfer to and from the working fluid and maximum conversion of the input heat to useful work by reducing thermodynamic losses as noted above. Until recently the heat transfer estimates used in reciprocating heat engines have been based on extrapolation of expressions that were developed for steady-pressure, steady-flow conditions. Because the ordinary, steady-state convective model contains no term to account for the oscillatory effect in variable volumes, designs of reciprocating engines are likely not based on these steady flow expressions. Using two Massachusetts Institute of Technology (MIT) test rigs (gas spring and gas springtheat exchanger) Kornhauser ${ }^{20}$ confirmed experimentally that there was a fundamental difference between steady and oscillatory flow heat transfer in the variable cylinder volumes of the MIT test rigs. Building on the work done by Lee, Smith, Faulkner, and Chafe ${ }^{6,13,21}$, Kornhauser started the development of expressions suitable for oscillating pressure and oscillating flow conditions. He closely integrated experiment with analysis to achieve useful results with good qualitative but limited quantitative success.

In this study, entropy-based second law post-processing analysis is employed to characterize the various thermodynamic losses inside a 3-space solution domain (gas spring + heat exchanger + regenerator) operating under conditions of oscillating pressure and oscillating flow. The 3 -space solution domain was adapted from the 2 -space solution domain (gas spring+heat exchanger) in Kornhauser's test rig ${ }^{20}$ by modifying the heat exchanger space to include a porous regenerator system. A thermal non-equilibrium model which assumes that the regenerator porous matrix and gas average temperatures can differ by several degrees at a given axial location and time during the cycle is employed. A survey of the porous-media literature supports the need for thermal non-equilibrium porous-media models for Stirling regenerators ${ }^{1,22,30,34}$. Numerical simulation results of the pressure, temperature, velocity and surface heat transfer variations in the 3-space domain are post processed for thermodynamic loss calculations. These simulation and loss calculation results are compared with similar results of an earlier study ${ }^{11,12}$ in order to evaluate the effect of adding a regenerator to the 2-space model. Also results of surface heat flux, temperature difference between the gas temperature at the radial center of the heat exchanger and the heat exchanger wall, temperature contours and velocity vectors are compared to relevant results from the literature.

Second-law analysis focusing on entropy generation has been playing a dominant role in recent times and has proven to be a useful tool in identifying the mechanisms and system components that are responsible for thermodynamic losses and for indicating how to minimize these losses in practical equipment in order to improve performance ${ }^{10}$. Entropy generation destroys part of the available work of a system and is associated with 
thermodynamic irreversibilities related to pressure drop, finite heat transfer, friction, mixing and other nonidealities within systems. Past studies have described various analytical and empirical techniques for entropy-based optimization of engineering systems. Bejan ${ }^{2,4,5}$ has focused on the different mechanisms behind entropy generation in applied thermal engineering. Numerous other investigations (mostly numerical) have been performed to determine entropy generation and irreversibility profiles for different geometric configurations, flow situations, and thermal boundary conditions ${ }^{24,25,28}$.

A valuable tool in the design of a high performance engine is a numerical code with multi-dimensional modeling capability and an ability to closely simulate the thermal-fluid processes inside the engine and account for all the thermodynamic losses. This study utilized 1-D Sage and 2-D Fluent commercial numerical codes to obtain computer models of the 3-space solution domain and to analyze and post-process the numerical simulation results. A thermodynamic loss post-processor is developed to characterize the major thermodynamic losses inside the 3-space model components.

Sage is a 1-D, multi-variable thermodynamic modeling package that supports simulation and optimization of spring-mass-damper resonant systems and Stirling-cycle coolers and engines. In the Sage code, an engineering system is built up from component parts obtained from component palettes. The components function as a whole by virtue of their connections which could be due to gas flow, heat flow, pressure, density, etc. Sage calculations are performed via its solver and optimizer routines.

Fluent is a state-of-the-art computational fluid dynamics (CFD) software package with comprehensive and flexible physical modeling and multi-physics capabilities for simulating fluid flow and heat transfer in complex geometries. The Fluent package includes the solver (FLUENT), the preprocessor (GAMBIT) for geometry modeling and mesh generation, an additional preprocessor (TGrid) that can generate volume meshes from existing boundary meshes and filters (translators) for import of surface and volume meshes from a variety of CAD/CAE packages. All functions (specifying problem type and numerical technique, setting boundary and initial conditions, defining fluid properties, etc.) are accessible in FLUENT through the Graphical User Interface (GUI) an interactive, menu-driven interface with many tools to compute a solution, visualize the flow physics, animate transient data sets, as well as to extract data for post processing the numerical results. Tools such as point, line, rake and surface are used to obtain the data of required parameters.

\section{B. Scope/Objectives}

The rest of this paper can be broadly categorized as:

- An Overview of the Stirling Engine

- Modeling of the 3-space Solution Domain

- Regenerator-Effect of change from Sage 2-space to Sage 3-space Modeling

- Thermodynamic Loss Modeling

- Numerical Simulation Results

- Thermodynamic Loss Post-Processing

- Conclusions and Suggestions for Future Work

In order to fully understand the fundamental objective of this research effort, it is necessary to provide an overview of the Stirling engine and its operation. A description of Kornhauser's MIT 2-space Test Rig is next presented so as to provide the proper context for the 3-space solution domain used this study. Following this, the 1-D Sage and 2-D Fluent computer modeling procedures for the 3-space solution domain are presented. After that, the effect of adding a regenerator to the 2-space solution domain in Kornhauser's MIT test rig is discussed. Next, conservation equations of mass, momentum and energy are united with the second law of thermodynamics to derive a complete set of entropy generation equations appropriate for thermodynamic loss modeling. The numerical simulation results of the fluid pressure, temperature, fluid velocity and surface heat transfer variations are presented and post-processed for thermodynamic losses. Finally, conclusions are drawn and suggestions for future work provided.

An important and primary objective of this study is the use of the second law analysis for the development and application of a thermodynamic loss post-processor to characterize the major thermodynamic losses inside the 3space model. It is anticipated that the experience gained from the thermodynamic loss analysis of the simple 3-space model can be extrapolated to more complex systems like the Stirling engine via the incorporation of loss postprocessors in Stirling engine analysis codes in order to improve its optimization capability. It is also anticipated that a thermal non-equilibrium model of the regenerator such as that presented here, when incorporated in Stirling engine analysis codes, will improve our ability to accurately model Stirling regenerators relative to current thermalequilibrium porous-media models. 


\section{An Overview of the Stirling Engine}

Figure 1 below shows a schematic representation of the Stirling Technology Demonstration Convertor (TDC), a 55-watt space power Stirling engine prototype developed by Infinia Corporation (formerly Stirling Technology Corporation (STC)). It is essentially a free piston machine that generates electric power from a thermal energy input. The convertor is of the gamma type, gas-coupled, and single-acting. It can be divided into two basic subsystems electro-mechanical and thermo-fluid.

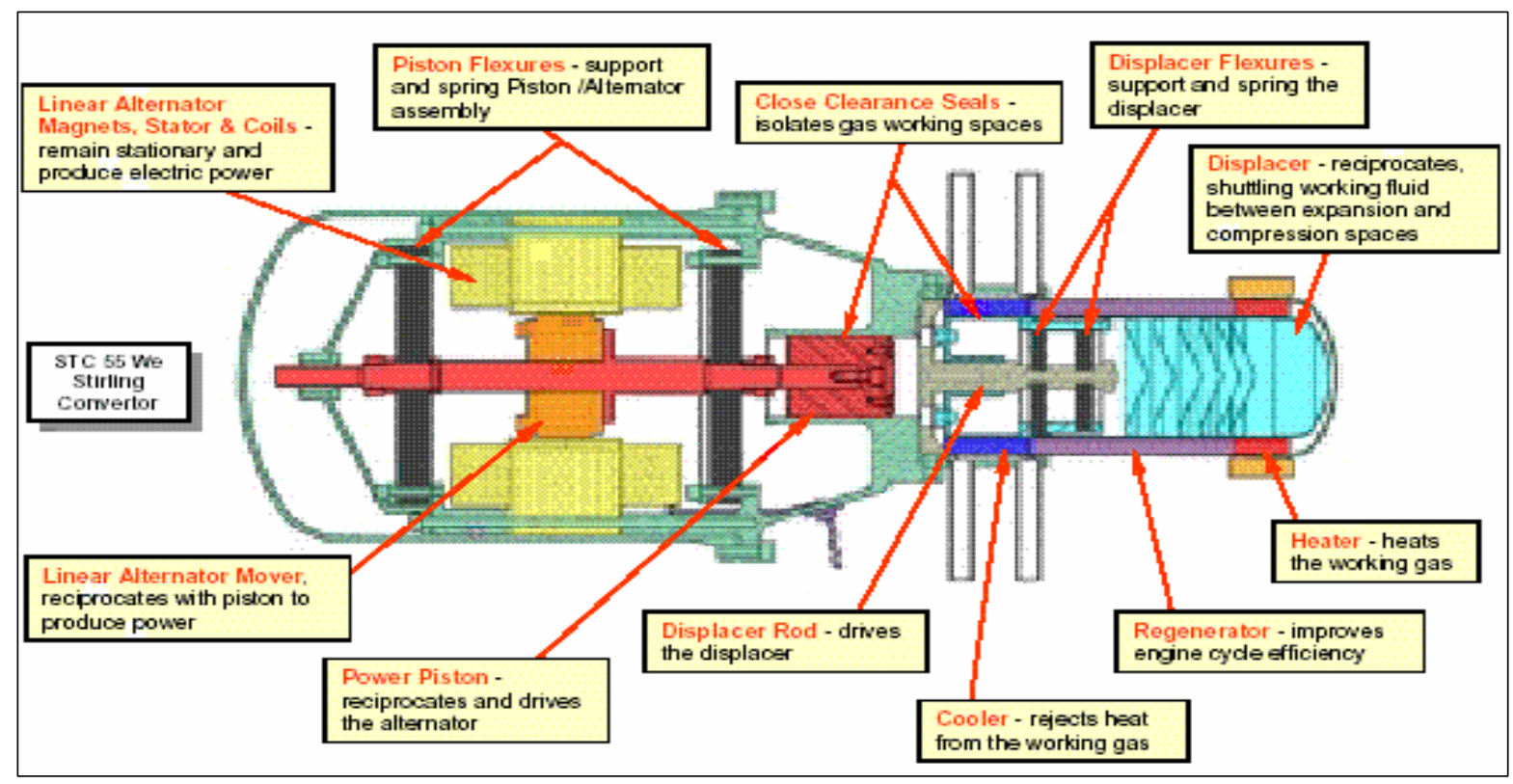

Figure 1. Schematic of STC's Stirling Convertor [Courtesy: www.nasa.gov].

The electro-mechanical subsystem consists of a pressure vessel containing a flexurally supported power piston/linear alternator assembly. The power piston separates the thermodynamic working space in the thermo-fluid subsystem to the right of the piston from the "bounce" space, a weak but measurable gas spring between the piston and the casing.

The thermo-fluid subsystem can be considered to have two distinct fluid circuits (internal and external) for most applications. The internal engine thermodynamic circuit is filled with working fluid (usually a gas: air, helium or hydrogen) at an elevated pressure and is comprised of a flexurally supported displacer/rod assembly, two variable volumes (compression and expansion volumes) and three heat exchangers in series: the heater, the regenerator and the cooler. The heater-head channels the heat from an external heat source, an electric heater (radioisotopes are the planned heat source for space application), into the expansion space and heats the working fluid to a temperature of about $650^{\circ} \mathrm{C}$. The regenerator is a duct packed with some porous matrix (solid containing interconnected pores for fluid flow). It is often made by stacking together a number of fine wire mesh screens, random fibers (for the TDC), foam metal plugs, or perforated disks or felts to form a kind of metallic heat sponge. The cooler, in contact with a heat sink, a water circuit in the laboratory (solid conduction to a radiator for space power applications), extracts excess heat from the compression space providing a cold-sink temperature of about $80^{\circ} \mathrm{C}$.

The external circuit (not shown in Figure 1) provides thermal energy which could come from just about any thermal energy source, available at a sufficiently high temperature. Stirling engines have been run on concentrated solar energy, thermal energy storage batteries, metal combustion, isotope nuclear energy, as well as a variety of liquid and solid fuels (oil, coal, gas, etc.).

Clearance seals used for the power piston and displacer isolate the gas working spaces. The radially-stiff flexures make tight clearance seals possible. The convertor uses hot-end materials capable of operating at the hotend temperature over the planned life of the machine.

The TDC operates in a closed regenerative thermodynamic cycle created in the thermo-fluid subsystem. During each working cycle, the power piston and the displacer reciprocate in a coordinated almost sinusoidal fashion (with 
the power piston motion lagging the displacer motion ${ }^{16}$ ), shuttling the gas at different temperature levels through the heat exchangers between the expansion and compression spaces. Pressures acting on each end of the displacer are only slightly different, relative to the mean pressure level. On the other hand, the relatively large "bounce" space to the left of the power piston (see Figure 1), compared to the working space volume (compression, expansion and heat exchanger volumes, etc), ensures that the pressure excursions in the bounce space will be substantially less than inside the engine working space. The larger pressure excursions inside the working spaces help drive the piston, alternately compressing and expanding the gas. The power piston's attached alternator parts oscillate within the alternator's magnetic field to produce electric power. The power piston extracts net power from the working space gas due to the larger pressure excursions on that side. The oscillating gas pressures within the working spaces, the spring-mass-damper systems of the piston and displacer, and the oscillating load on the piston produced by interaction with the alternator, all interact to produce sustained oscillating motion of the piston and displacer-when engine geometries, piston and displacer masses and heat sources and sinks are appropriately chosen.

The action of the Stirling engine regenerator is most easily understood by first imagining the gas behavior without a regenerator. In the absence of the regenerator, hot gas would be transferred by the action of the displacer directly from the expansion space/heater into the cooler/compression space, where it would have to be cooled. The heat extracted during the cooling would be rejected and lost. When the gas is subsequently returned to the expansion space, it would have to be reheated drawing more heat from the heat source. Extra heat would therefore be added and rejected during the cycle, with a consequence of loss of efficiency. With the regenerator in place, the hot gas gets cooled gradually as it passes from the hot end to the cold end, by giving up the heat to the regenerator solid matrix and therefore the gas leaves the regenerator already cooled, minimizing the heat to be rejected in the cold space. On the return journey, the gas is gradually heated up as it moves up the temperature gradient towards the expansion space, by picking up heat from the solid matrix that was deposited during the previous cycle. Thus the gas emerges into the heater with considerable heat already added, minimizing the heat to be added by the external source $^{24}$. The regenerator thus serves as an economizer for storing heat during one part of the engine cycle and for re-use during another part. The inclusion of an effective regenerator results in a substantial increase in Stirling engine efficiency and is necessary for the Stirling engine to be of practical use.

Extensive efforts have been focused on improvement in regenerator technology. These efforts have been categorized into areas of materials and geometry, numerical modeling, and experimental measurement ${ }^{17,23,29,32,35}$. A NASA regenerator research grant effort led by Cleveland State University, with subcontractor assistance from the University of Minnesota, Gedeon Associates, and Sunpower Inc. has been providing computational and experimental results to support definition of various empirical parameters and "closure" relations needed in defining a thermal, non-equilibrium, macroscopic, porous-media model for use in multi-D Stirling codes for regenerator simulation $^{33}$. 


\section{Modeling of the 3-space Solution Domain}

(Kornhauser's MIT 2-space Solution Domain Modified).

\section{A. Kornhauser's MIT 2-space Test Rig}

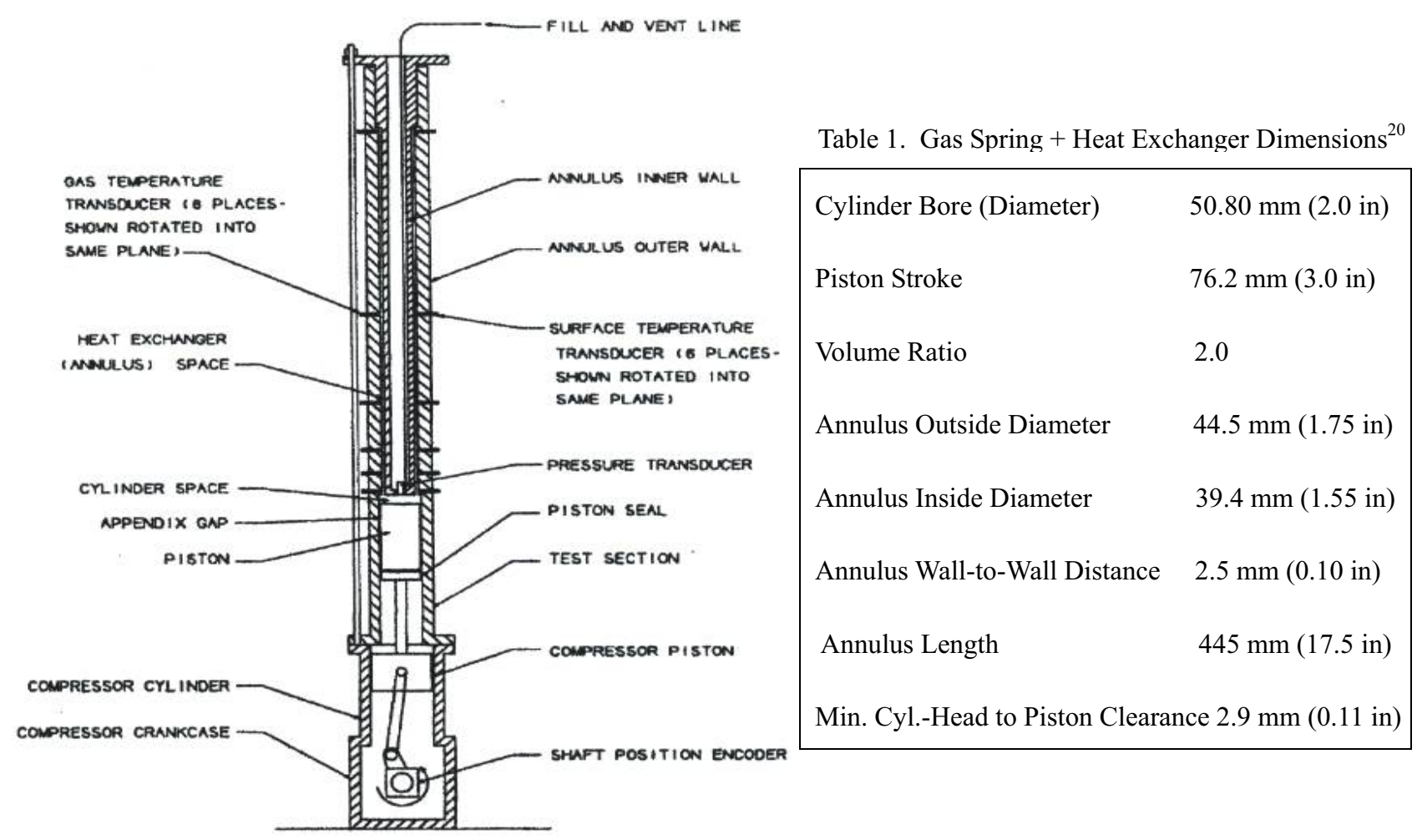

Figure 2. Kornhauser's MIT Cylinder + HXer Test Rig.

Figure 2 shows a schematic representation of Kornhauser's piston-cylinder+heat exchanger test rig. A pistoncylinder device is mounted on a compressor base. The compressor piston drives the piston in the $50.8 \mathrm{~mm}(2.00 \mathrm{in}$.) diameter cylinder. The piston is sealed to the cylinder with a buna-n O-ring located more than a stroke's length from the piston face so that frictional heating of the cylinder wall would only affect results minimally. The piston top surface is brass, while the cylinder wall and head are made of steel. The fixed cylinder head has an annular opening leading into an annular dead-ended heat exchanger space such that flow can continuously pass between the heat exchanger and cylinder as the cylinder piston expands and compresses the gas similar to that in a Stirling engine compression space volume. The test section consists of the gas spring + heat exchanger space. A piston stroke length of $76.2 \mathrm{~mm}$ (3.00 in.) and volume ratio of 2.0 are used in this study. A volume ratio is defined to be the maximum cylinder volume (piston at "bottom-dead-center" (BDC)) over the minimum cylinder volume (piston at "top-deadcenter" (TDC)).

The heat exchanger annulus is $44.5 \mathrm{~mm}$ (1.75 in) outside diameter and $39.4 \mathrm{~mm}$ (1.55 in) inside diameter, for a wall-to-wall distance of $2.5 \mathrm{~mm}(0.10 \mathrm{in})$. The annulus is $445 \mathrm{~mm}(17.5 \mathrm{in})$ long, so that a volume ratio of 2.0 for the combined cylinder and heat exchanger resulted in a very small cylinder clearance volume; the cylinder-head distance is nominally $2.9 \mathrm{~mm}(0.11 \mathrm{in})$ at top center position. The heat exchanger entrance has the same crosssectional dimensions as the heat exchanger itself, the entrance corners being as sharp as could easily be machined. The inner wall of the heat exchanger space is made of steel. The outer wall of the heat exchanger space is steel lined with a $1.98 \mathrm{~mm}$ (0.078 in) layer of bronze-filled TFE (Teflon). This lining was chosen for thermal properties matching those of the Pyrex glass substrate of the surface temperature transducers. Because of the extended heat transfer surface in the annular heat exchanger, the energy flows are more complicated than in a simple gas spring. 
The apparatus is belt driven by a D.C. motor to allow for speed adjustment. The apparatus fill line is a capillary tube of negligible volume. The gas spring + heat exchanger dimensions are tabulated in Table 1.

The 3-space model solution domain (gas spring + heat exchanger + regenerator) was adapted from the 2-space model solution domain (gas spring+heat exchanger) for Kornhauser's 2-space test rig (Figure 2) by modifying the heat exchanger space to include a porous regenerator matrix. 1-D Sage and 2-D Fluent commercial numerical codes are used to model, analyze and post-process the thermal-fluid phenomena inside the 3-space domain. The modeling procedures using Sage and Fluent are presented in subsections III.A.1 and III.A.2 below. Note that in Figures 3 (Sage model) and 4 (Fluent model), the regenerator is shown located at the heat exchanger end opposite from the end adjacent to the cylinder. The justification for this is discussed in Section IV. Note also that this 3-space device is different from the usual Stirling engine. The regenerator is not located between a heater and cooler as in a Stirling engine. Also, whereas the 3-space device contains a single variable volume on one side of a heat-exchangerregenerator arrangement, the Stirling engine contains a variable volume on each side of a cooler-regenerator-heater heat-exchanger circuit. The flow dynamics in the 3-space solution domain is expected to approximate some of the flow dynamics in the Stirling engine thermo-fluid subsystem

\section{Sage 3-space Model (1-D)}

Figure 3 is a representation of the Sage graphical interface illustrating the 1-D model of the 3-space solution domain.

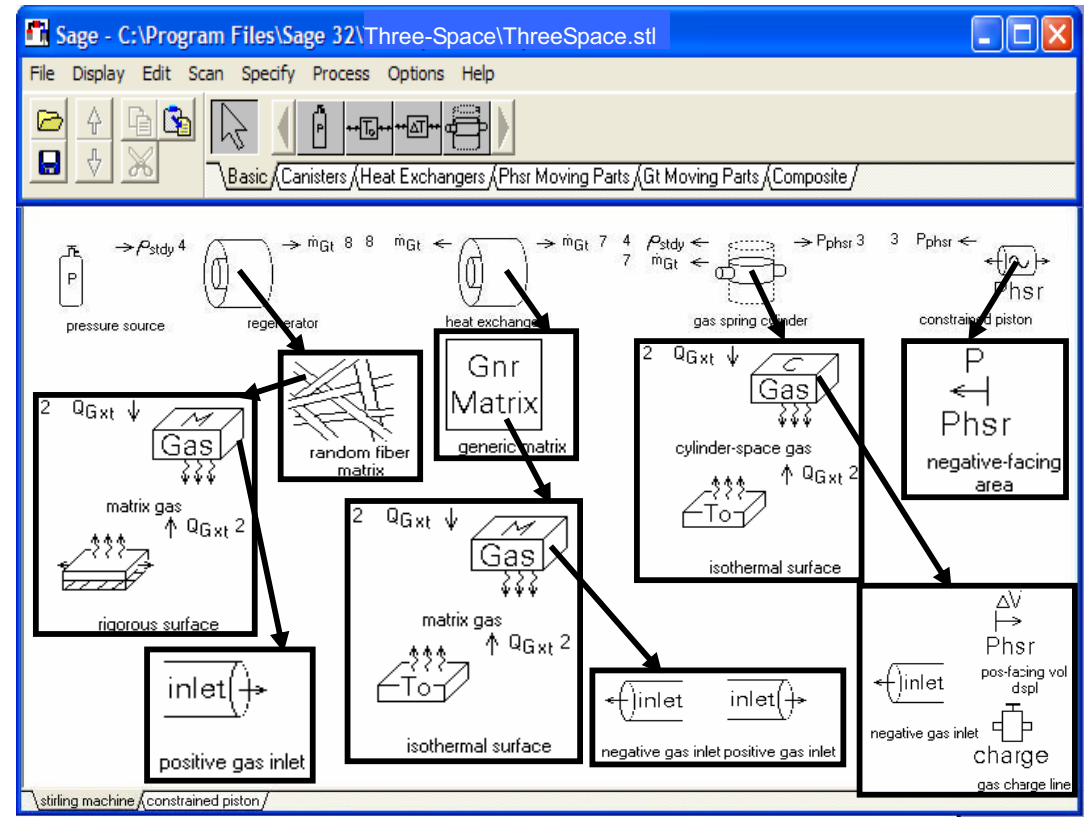

Figure 3. Sage Model of the 3-space Solution Domain.

Sage provides 8 menus - File, Display, Edit, Scan, Specify, Process, Options, and Help - each with selfexplanatory commands and graphical edit windows with model-component palettes. When "show window" in the edit menu is activated, the highest level graphical edit window (Stirling machine window) and its model-component palettes appear. Each model-component palette contains buttons arranged in tab-selected pages corresponding to different categories of model components. Figure 3 shows the Stirling machine window with six Stirling engine model component palettes - "Basic", "Canisters", "Heat Exchanger", "Phasr Moving Parts", "Gt Moving Parts" and "Composite". A Sage model is, in part, a collection of component parts assembled in a graphical edit window by clicking and dragging the components into the window and connected in a particular way to form a complete system. Model components are organized in a hierarchical structure. The root model component contains a number of sub-components which may themselves contain sub-sub-components. Child (sub) models are accessed by doubleclicking on parent models. The labeled arrows sticking out of the sides of components are boundary connectors. They are joined together as matched pairs with numbers $(2,3,4$, etc.) identifying the match. The labels are meant to 
suggest what information is transferred across the connection between components. Sage is also able to perform such functions as open and save files, increase/decrease connector level, copy, cut and paste components.

The 3-space solution domain (gas spring + heat exchanger + regenerator) is modeled using Sage's Stirling machine model component palettes. The variable volume cylinder space in Fig. 2 and the charge pressure inside it are modeled in the Sage code using a generic cylinder (renamed "gas spring cylinder" in Fig. 3) and a pressure source, both obtained from the "Basic" component palette at the Stirling machine level. The pressure source comes with a built in steady-state density connection $\left(\rho_{\text {stdy }}\right)$ and acts as an isobaric gas reservoir in that the density in the gas domain adapts itself so that the time average pressure is continuous across the connection. The cylinder-space gas and isothermal surface, child models of the gas spring cylinder, are obtained from the "Gas Domain" and "Cylinder Walls" component palettes respectively at the gas spring cylinder level. The arrows labeled 2 in the gas spring edit window indicate a space-time varying heat flow connection $\mathrm{Q}_{\mathrm{Gxt}}$ between the $\mathrm{z}$-face of the cylinder-space gas and the isothermal surface. The connection arrows from the positive-facing volume displacement phasor, gas charge line and negative gas inlet, all child models of the cylinder-space gas, are moved up to the Stirling machine level for pressure connections $\left(\mathrm{P}_{\text {phss }}\right)$ to the constrained piston (label 3), density connections $\left(\rho_{\text {stdy }}\right)$ to the pressure source (label 4) and mass flow connection to the heat exchanger (label 7) respectively. The positive-facing volume displacement phasor represents the volume change of the gas space due to the motion of the piston. The gas charge line and negative gas inlet are obtained from the "Charge/Inlet" component palette in the cylinder-space gas level.

The piston in Figure 2 is modeled using the constrained piston obtained from the "Phsr Moving Parts" component palette at the Stirling machine level. Its child model, the negative facing-area phasor, is obtained from the "Mechanical Attachment" component palette in the constrained piston level. Its pressure connection arrow is moved up to the Stirling machine level for connection there to the gas spring cylinder (label 3).

The annular canister in Figure 3 is used to model the heat exchanger and the regenerator. It is obtained from the "Canisters" component palette in the Stirling machine level. The generic matrix, child model of the heat exchanger, represents the internal structure of the heat exchanger. It is obtained from the "Matrices" component palette in the annular canister level. The matrix gas and isothermal surface, child models of the generic matrix, are obtained from the "Gas Domain" and "Matrix Solids" component palettes respectively in the generic matrix level. The arrows labeled 2 in the generic matrix edit form indicate a heat flow connection between the matrix gas and the isothermal surface. The negative and positive gas inlets, child models of the matrix gas, are obtained from the "Charge/Inlet" component palette in the matrix gas level. The mass flow connection arrows from the negative and positive gas inlets are moved up to the Stirling machine level for connection there to the regenerator (label 8) and gas spring cylinder (label 7). The regenerator is modeled in a similar manner as for the heat exchanger except for the requirement of a rigorous surface child model for the surface condition of the random fiber matrix representing the internal structure of the regenerator. The positive gas inlet, child model of the matrix gas, models the gas flowing from the regenerator into the heat exchanger (label 8). We see from Figure 3 and the foregoing descriptions that the 3-space model components communicate with each other using density $\left(\rho_{\text {stdy }}\right)$, mass flow $\left(\dot{\mathrm{m}}_{\mathrm{Gt}}\right)$, heat flow $\left(\mathrm{Q}_{\mathrm{Gxt}}\right)$ and pressure $\left(\mathrm{P}_{\mathrm{phsr}}\right)$ boundary connections.

Once a model structure is created in Sage, numerical inputs for the model component are either specified or modified. Sometimes user-defined variables (special output variables) can be added to model components. Sage numerical simulation is initiated after initializing component and overall model parameters. Sage calculations are performed via its solver and optimizer routines.

\section{Fluent 3-space Model (2-D)}

The Fluent 2-D model of the 3-space solution domain is illustrated in Figure 4 below. The origin $(0,0)$ of the $x-y$ coordinate system is located at the intersection of the cylinder head and the line of symmetry. The Fluent code exploits the symmetry of the problem domain to model only one-half of the domain. The geometry of the physical domain of interest is represented within the preprocessor GAMBIT using fundamental geometric entities (points, lines, arcs, circles, curves, splines and surfaces) which can be manipulated (translated, rotated, scaled, projected, split, joined, etc.) as desired. Grid generation, the process of discretizing the problem domain with individual cells over which the flow equations are integrated, follows the geometric representation of the domain of interest. The locations of the corner points of these cells constitute the "grid" or "mesh" which is stored in a data base. Fluent provides complete mesh flexibility, including structured and unstructured meshes, different mesh types and ability to refine and coarsen the grid based on the flow solution. The power law grid distribution type is used to obtain a finer grid at the boundaries of the model for more accurate resolution of the flow features. The baseline grid size $100 \times 20$ is presented in Figure 4 for illustration. The optimum linear dimension of the regenerator was determined via a systematic parameter analysis using the Sage code. This is discussed in Section IV. 


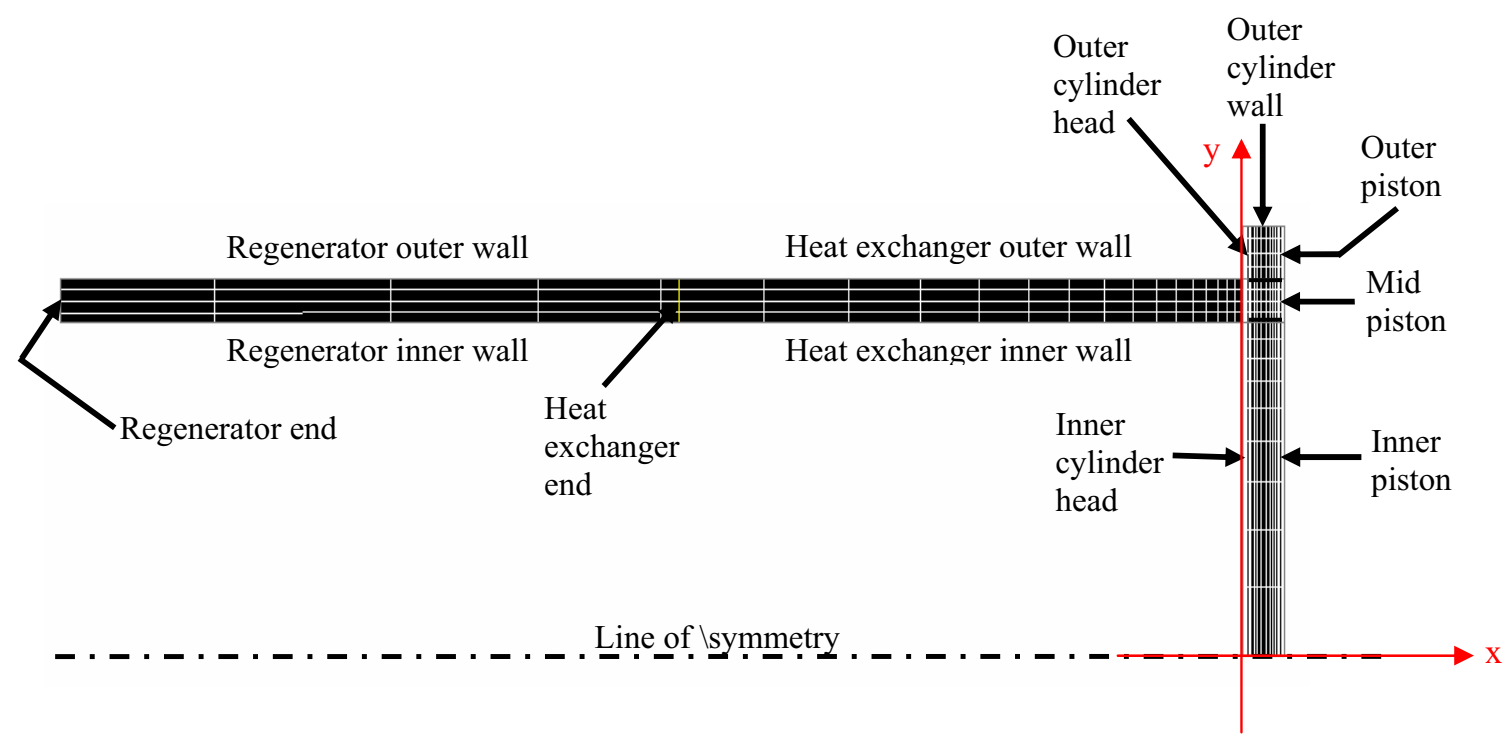

Figure 4. Fluent Model of the 3-space Solution Domain (Grid size: 100 x 20).

After modeling is completed in GAMBIT, the meshed geometry is exported to Fluent where the problem is completely formulated using an interactive, menu-driven Graphical User Interface (GUI). Problem formulation involves specifying the problem type, model options, volume conditions, boundary conditions, initial conditions, formulation type (implicit or explicit), the numerical technique needed to solve the flow equations and solution controls. The instantaneous position $\mathrm{X}_{\mathrm{p}}(\mathrm{t})$ and velocity $\mathrm{U}_{\mathrm{p}}(\mathrm{t})$ of the piston face in the $\mathrm{x}$-direction are defined as

$$
\begin{aligned}
& X_{p}(t)=L_{c}+\frac{S}{2}\left[1+\sin \left(\omega t+\frac{3 \pi}{2}\right)\right] \\
& U_{p}(t)=\frac{d X_{p}(t)}{d t}=\frac{S}{2} \omega \cos \left(\omega t+\frac{3 \pi}{2}\right)
\end{aligned}
$$

where parameter $\mathrm{L}_{\mathrm{c}}$ is the length of the cylinder clearance volume when the piston is at TDC, $\omega$ is angular frequency, $\mathrm{t}$ is time and $\mathrm{S}$ is the piston stroke. Fluent uses the velocity equation. A user defined function (UDF) is written to input the velocity equation into Fluent. After problem formulation, simulation is performed using the FLUENT flow solver module. 


\section{Regenerator-Effect on Change from Sage "2-space" to "3-space" Modeling}

The results of a prior study ${ }^{12}$ of a Sage model of the MIT 2-space solution domain (Figure 5(a)) indicated a cylinder-cooling effect, with heat being drawn from the environment through the cylinder walls into the cylinder volume and pumped from the heat exchanger gas volume through the heat exchanger cylinder walls into the environment. Since the 3-space solution domain used in this study was adapted from the MIT 2-space solution domain by modifying the heat exchanger space to include a porous regenerator system, it was decided to investigate the regenerator impact on the cylinder-cooling effect in changing from Sage 2-space to 3-space modeling. Table I summarizes the results of the investigation.

The second row of Table 1 shows results obtained using a Sage model of the original MIT 2-space solution domain. This model assumed isothermal wall temperatures of $294 \mathrm{~K}$ for both the heat exchanger and the cylinder. Since the purpose of a "real cooler" is to reduce the temperature of the cooled area relative to the environment, the temperature of the cylinder walls was reduced (with everything else remaining the same) over a range of temperatures below $294 \mathrm{~K}$, in order to obtain the wall temperature below which no cooling results. The effects of changing the cylinder wall temperature are shown in Runs 1 thorough 5 (Table 1). Based on these results, the cylinder temperature $(289 \mathrm{~K})$ for the next series of runs was established in Run 3. Run 3 (also designated Run A) was thus chosen to be the reference "cooler" case for subsequent runs B through H.

Next, in preparation for converting part of the $44.5 \mathrm{~cm}$ long heat exchanger into a regenerator, the heat exchanger was first split arbitrarily into two parts $(40 \mathrm{~cm}$ part and $4.5 \mathrm{~cm}$ part) such that of the 16 equilength cells in the $44.5 \mathrm{~cm}$ long heat exchanger, 14 equilength cells are now contained in the $40 \mathrm{~cm}$ part and 2 equilength cells in the $4.5 \mathrm{~cm}$ part sectioned out from the end of the heat exchanger facing the cylinder for use as the regenerator. With no regenerator porous matrix added, Run B was then initiated with ensuing results essentially the same as for Run A. The slight differences are likely due to the non identical equilength cells.

The $4.5 \mathrm{~cm}$ part of the heat exchanger was next converted into a regenerator resulting essentially in a 3 -space Sage model of the solution domain - cylinder, regenerator and heat exchanger. The regenerator matrix is modeled as either a generic random-fiber or a "dedicated" random-fiber. The regenerator wall is adiabatic and heat exchanger walls is kept fixed at $294 \mathrm{~K}$. The regenerator position relative to the heat exchanger and cylinder is now varied for best possible location based on cooling performance. With the regenerator between the heat exchanger and the cylinder (Figure 5(b); Runs C and D, Table 1) the cooling effect of the device was completely "wiped out", apparently because the regenerator acted as a "heat dam" to prevent heat from moving from the cylinder to the heat exchanger. Runs C and D differ only in the representations of the $90 \%$ porosity regenerator matrix (generic-matrixbased random-fiber model in Run C, and "dedicated" random-fiber model for Run D).

In Runs E and F (90\% porosity generic-matrix-based random fiber model and dedicated 90\% porosity randomfiber model, respectively), the regenerator was placed at the end of the heat exchanger away from the cylinder (Figure 5(c)). A significant improvement in cooling performance, relative to the performance of the original 2-space Sage model (Run A) and Run B was noted. The coefficient of performance (COP) improved from $\sim 0.145$ to $\sim 0.211$. It is also observed that the switch from generic matrix based random fiber to "dedicated" random fiber matrix (Run $\mathrm{C}$ to Run D and Run E to Run F) has less effect with the regenerator on the heat exchanger end away from the cylinder (Runs E and F) than with the regenerator between the heat exchanger and the cylinder (Runs C and D) perhaps because "less is going on" at the heat exchanger end away from the cylinder.

In Run $\mathrm{G}$, the impact of the $90 \%$ porosity matrix at the end of the heat exchanger opposite the cylinder was checked by removing the generic matrix based $90 \%$ porosity random fiber matrix. Very similar results to Runs A and B were obtained, as anticipated.

In Run $\mathrm{H}$, it was thought necessary to investigate if the improvements shown in Runs $\mathrm{E}$ and $\mathrm{F}$ - regenerator at end of heat exchanger away from cylinder — were due primarily to the $10 \%$ reduction in the volume produced by adding the $90 \%$ porosity random fiber. This investigation was done by simply cutting off $4.5 \mathrm{~cm}$ of the heat exchanger. Removing this $4.5 \mathrm{~cm}$ 's "worth of volume" does improve the cooling performance relative to Runs A and $\mathrm{B}$ (from COP $\sim 0.146$ to $\mathrm{COP} \sim 0.153$ ), but not nearly as much as putting a $90 \%$ porosity random fiber matrix in the $4.5 \mathrm{~cm}$. part of the heat exchanger away from the cylinder (COP $\sim 0.146$ to COP $\sim 0.211)$. Thus the impact of the regenerator matrix on the cooling performance is significant but it is not presently clear why the addition of the regenerator matrix at this end improves the performance. Since the regenerator is not located between a heater and a cooler, as in a Stirling engine, it is not as obvious how storage and release of heat to and from the regenerator matrix is improving the cooling efficiency. 


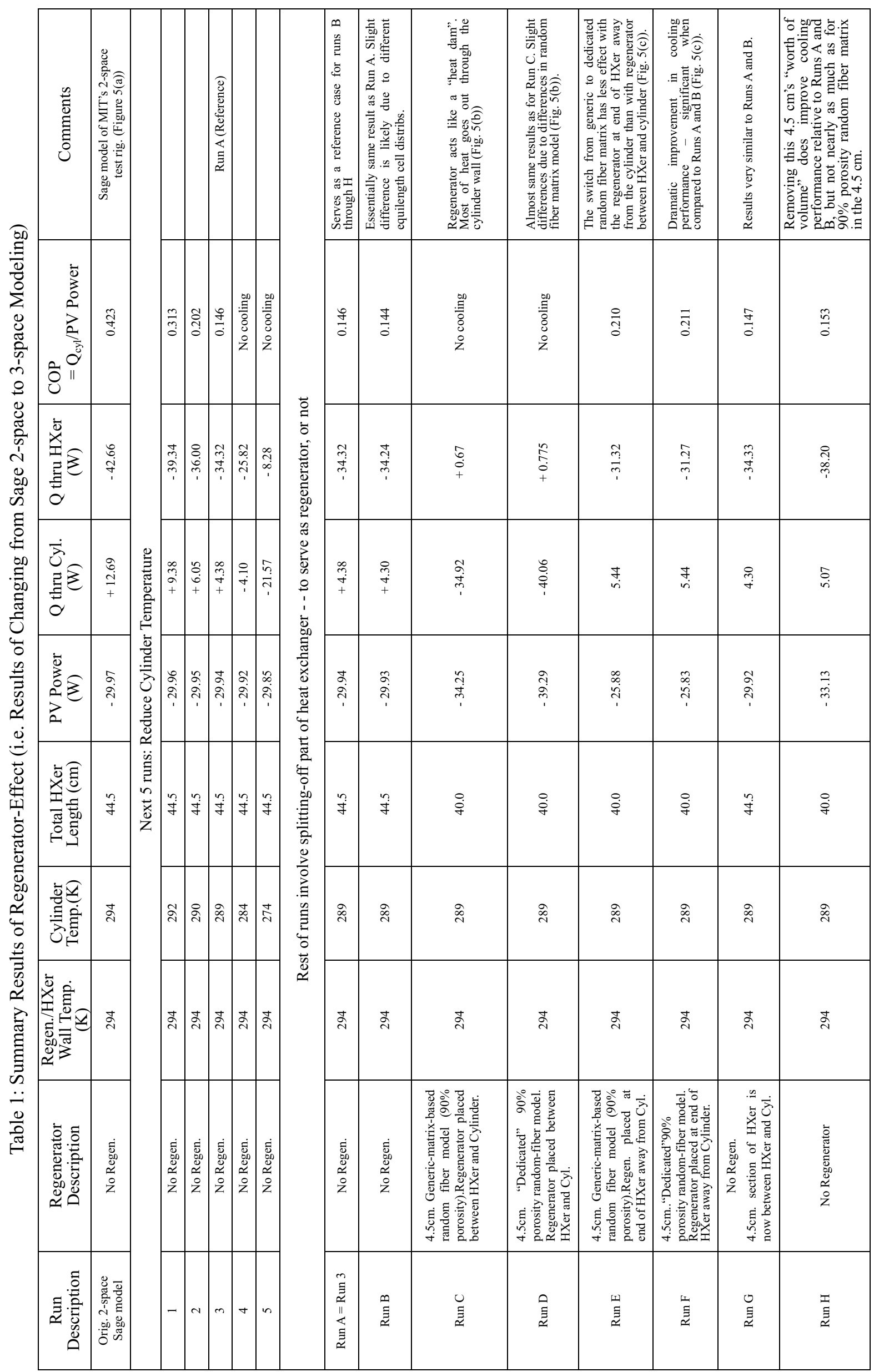




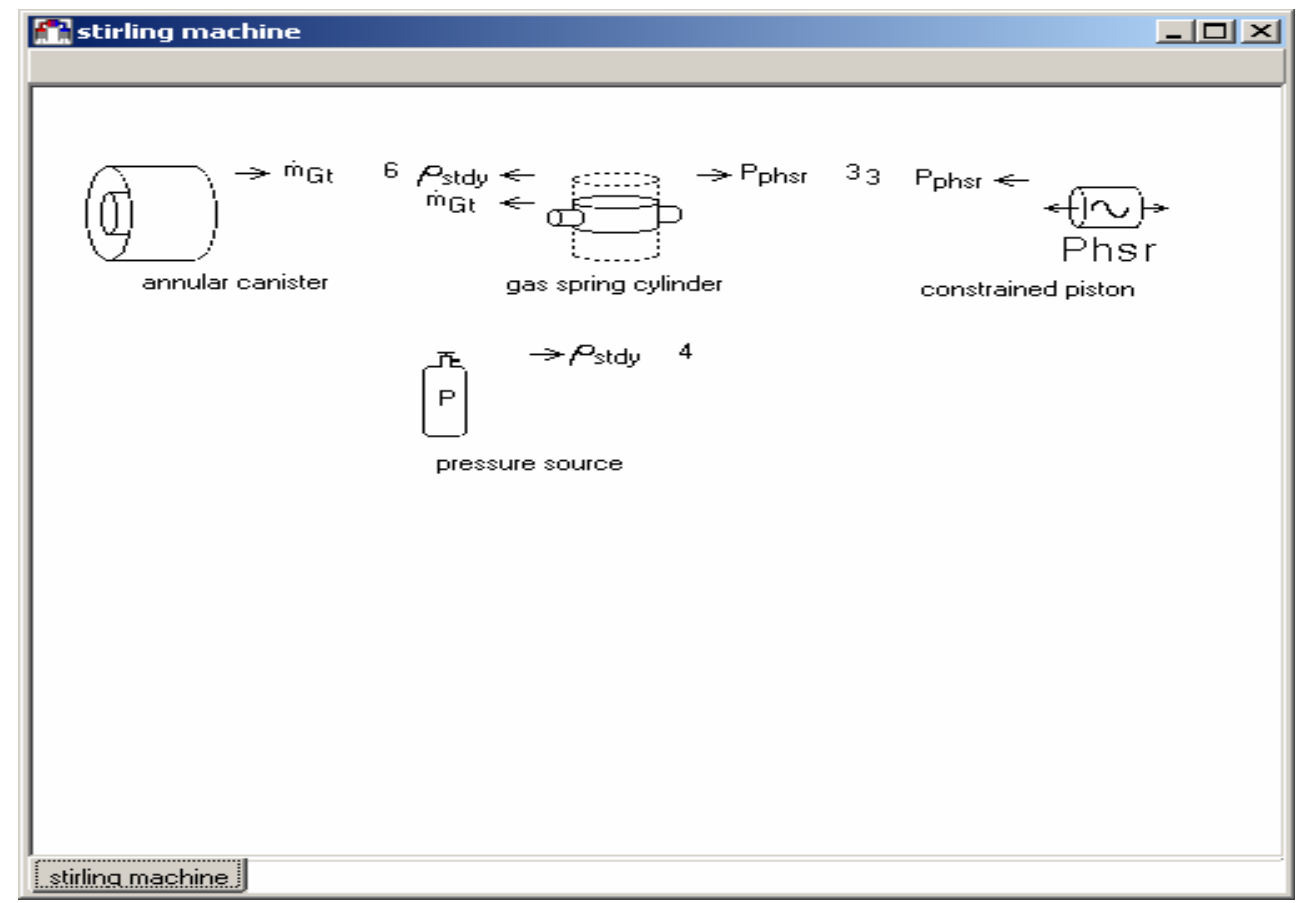

Figure 5(a): Sage Model Schematic of Original 2-space MIT Test Rig

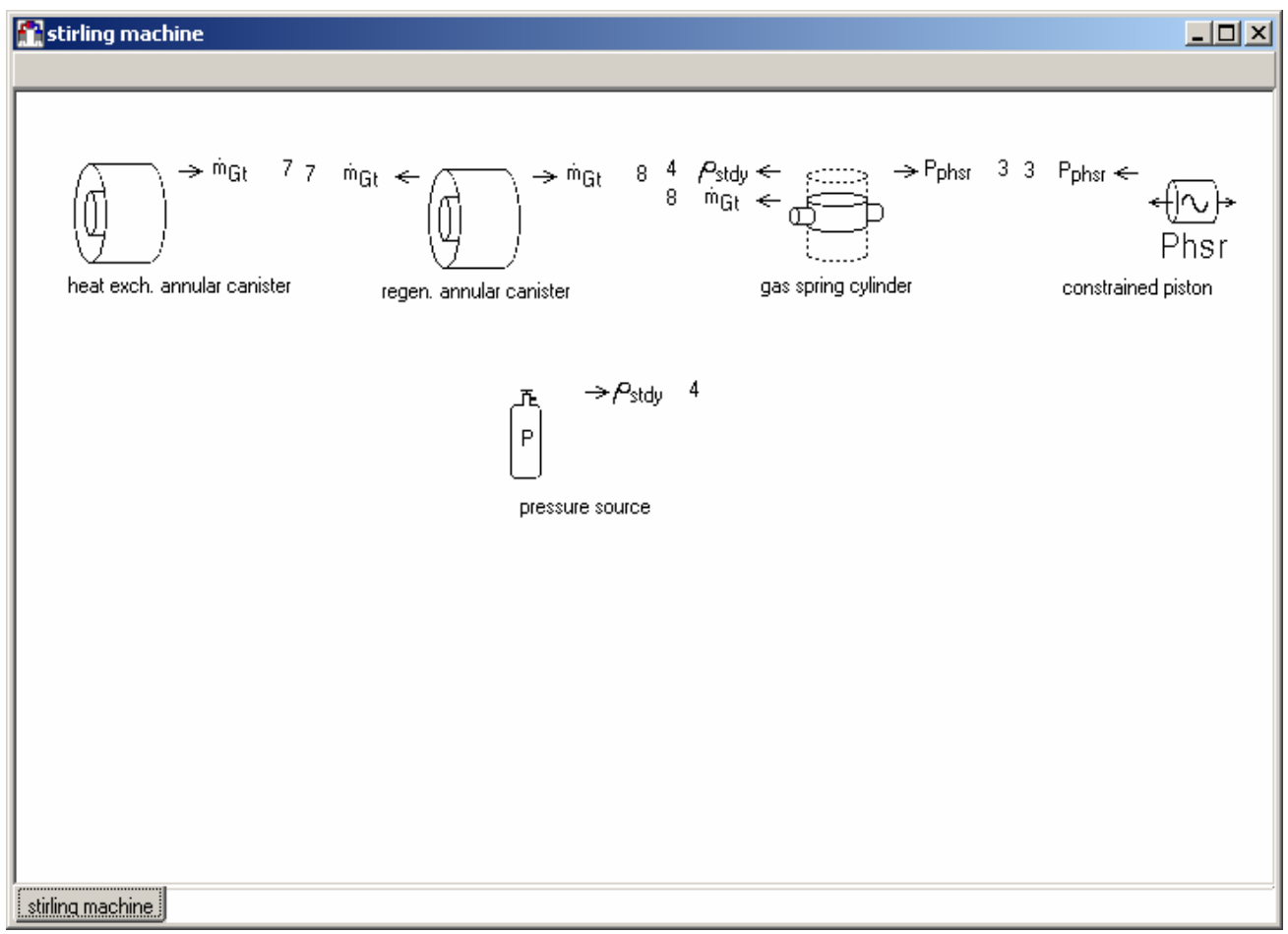

Figure 5(b): Sage Model Schematic Corresponding to Runs C and D 


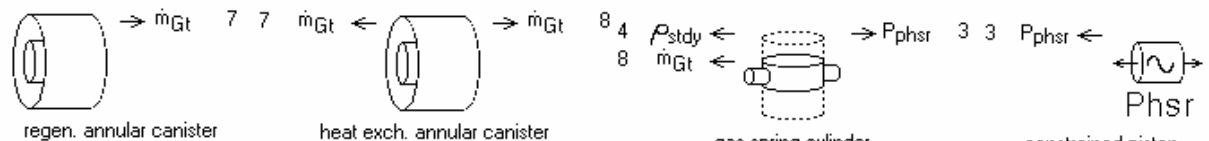

Figure 5(c): Sage Model Schematic Corresponding to Runs E and F

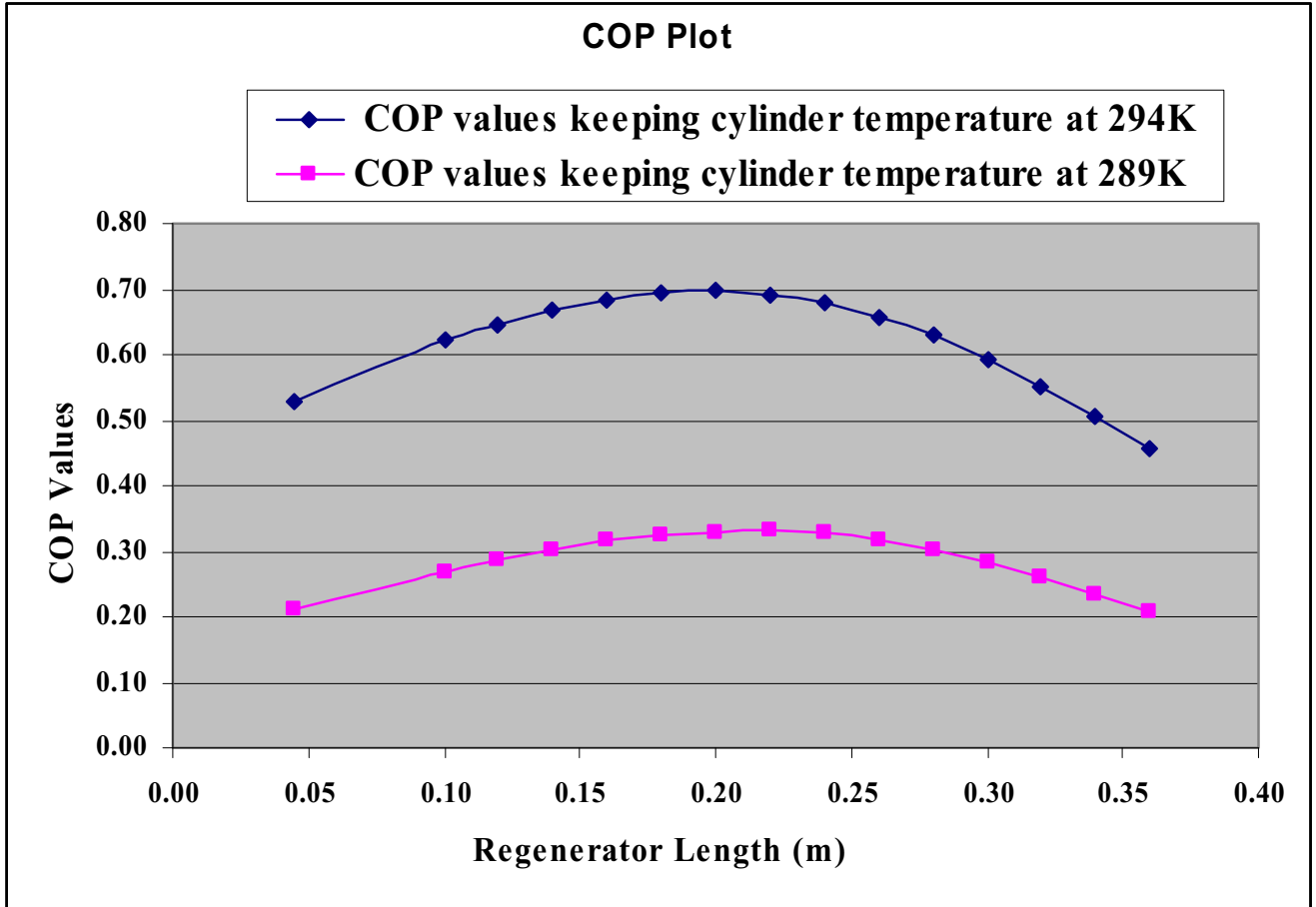

Figure 6: COP vs. Regenerator Length (m) 
With the 3-space model configured as in Runs E and F, a series of parameter optimization studies can be designed and executed in an effort to obtain optimum values for regenerator parameters such as regenerator length, wire diameter, annulus diameter (inner and outer) and porosity with the objective being the maximization of the cylinder-cooling effect. The Run F configuration of the 3-space model is used in this study because of its slight COP advantage over the Run E configuration (see Table 1). The results of coefficient of performance (COP) values over a range of regenerator lengths, which appears to be one of the easiest optimizations to do, are presented in graphical form in Figure 6. The variation in the regenerator length is made by increasing its length from $4.5 \mathrm{~cm}$. while ensuring that the overall length of the regenerator-heat exchanger domain remains constant at $44.5 \mathrm{~cm}$. The optimum cylinder-cooling effect $(\mathrm{COP}=0.33019)$ is observed at the regenerator length of $22 \mathrm{~cm}$. Other regenerator parameter optimization studies will be left for some future considerations.

In order to observe the effect of the regenerator on the numerical simulation results of the original 2-space model $^{12}$, the 3-space model operating conditions were set to match those of the 2-space model (201.7 RPM, 1.008 $\mathrm{MPa}$., $\left.\mathrm{T}_{\text {wall }}=294 \mathrm{~K}\right)$. For this set-up, the best possible cylinder-cooling effect $(\mathrm{COP}=0.69647)$ is observed at a regenerator length of $20 \mathrm{~cm}$. as illustrated in Figure 6 . The optimum length of the regenerator is thus set at $20 \mathrm{~cm}$. for the remaining numerical simulations to be conducted with the cylinder wall temperature of $294 \mathrm{~K}$. A discussion of the effect of the regenerator on the numerical simulation results is presented in Section V. 


\section{Thermodynamic Loss Modeling}

The fluid flow in the 3-space domain is highly compressible due mainly to internal volume changes. The competing effects of the heat transfer due to temperature gradients and flow friction points to thermodynamic loss models which require the coupling effects of the mass, momentum and energy conservation equations be united with the concept of entropy generation which relies on the second law of thermodynamics.

\section{A. Local or Microscopic Equations}

The theoretical development of the thermodynamic loss models begins with a set of generalized local or microscopic conserved equations of mass, momentum and energy and non-conserved entropy equation for the two mechanically distinct phases, $\alpha$ (flowing fluid) and $\beta$ (stationary solid) inside the non-porous regions of the 3 -space domain. It is assumed that the $\alpha$ and $\beta$ phases do not react chemically; that the fluid phase is assumed to behave as a linearly viscous fluid (Newtonian) whose properties are known functions of temperature and pressure, or other combinations of state properties; that the density of the solid is constant and that the no-slip assumption at the fluidsolid interface is valid. The following local or microscopic equations of mass, momentum, energy and entropy that govern the flow and describe the losses in the non-porous regions of the 3 -space domain are similar to those derived in any fluid mechanics text.

\section{Conservation of Mass Equation}

The conservation of mass equation, applicable only to the $\alpha$-phase, is:

$\frac{\partial \rho_{\alpha}}{\partial \mathrm{t}}+\vec{\nabla} \cdot\left(\rho_{\alpha} \overrightarrow{\mathrm{u}}_{\alpha}\right)=0$

This equation is a scalar equation with 4 unknowns: the gas density $\rho_{\alpha}$ and three components of the gas velocity $\vec{u}_{\alpha}$.

\section{Conservation of Momentum Equations}

The conservation of momentum equation is also applicable only to the $\alpha$-phase. The differential statement of the conservation of momentum for a Newtonian fluid with no body forces can be written as:

$$
\begin{aligned}
\frac{\partial\left(\rho_{\alpha} \overrightarrow{\mathrm{u}}_{\alpha}\right)}{\partial \mathrm{t}}+\vec{\nabla} \cdot\left(\rho_{\alpha} \overrightarrow{\mathrm{u}}_{\alpha} \overrightarrow{\mathrm{u}}_{\alpha}\right)+\vec{\nabla} \mathrm{p}_{\alpha}-\frac{1}{3} \vec{\nabla}\left(\mu_{\alpha} \vec{\nabla} \cdot \overrightarrow{\mathrm{u}}_{\alpha}\right)-\left(\vec{\nabla} \cdot \mu_{\alpha} \vec{\nabla}\right) \overrightarrow{\mathrm{u}}_{\alpha} \\
+\frac{2}{3}\left(\vec{\nabla} \mu_{\alpha}\right) \vec{\nabla} \cdot \overrightarrow{\mathrm{u}}_{\alpha}-2\left(\vec{\nabla} \mu_{\alpha} \cdot \vec{\nabla}\right) \overrightarrow{\mathrm{u}}_{\alpha}-\left(\vec{\nabla} \mu_{\alpha} \mathrm{x} \vec{\Omega}_{\alpha}\right)=0
\end{aligned}
$$

The parameter $\vec{\Omega}_{\alpha}$ is the vorticity vector. This equation is a vector equation with three components corresponding to the three components of velocity. The conservation of momentum equation produces 1 additional unknown, the fluid pressure, $\mathrm{p}_{\alpha}$. The fluid dynamic viscosity, $\mu_{\alpha}$, is a material property. For many fluids, $\mu_{\alpha}$ depends significantly on temperature and when appreciable temperature differences exist in the flow field it is necessary to regard $\mu_{\alpha}$ as a function of position.

\section{Conservation of Energy Equation}

The energy equation is applicable to both the $\alpha$ and $\beta$ phases.

$$
\begin{array}{c|c}
\alpha \text {-phase } & \beta \text {-phase } \\
\frac{\partial\left(\rho_{\alpha} \hat{\mathrm{u}}_{\alpha}\right)}{\partial \mathrm{t}}+\vec{\nabla} \cdot\left(\rho_{\alpha} \overrightarrow{\mathrm{u}}_{\alpha} \mathrm{h}_{\alpha}-\mathrm{k}_{\alpha} \vec{\nabla} \mathrm{T}_{\alpha}\right)=0 \quad \text { (5a) } & (\rho \mathrm{c})_{\beta} \frac{\partial \mathrm{T}_{\beta}}{\partial \mathrm{t}}-\vec{\nabla} \cdot\left(\mathrm{k}_{\beta} \vec{\nabla} \mathrm{T}_{\beta}\right)=0
\end{array}
$$

\section{$\beta$-phase}

The energy equations introduce 3 additional unknowns; the fluid and solid temperatures $\left(\mathrm{T}_{\alpha}\right.$ and $\left.\mathrm{T}_{\beta}\right)$, and the fluid specific internal energy $\hat{\mathrm{u}}_{\alpha}$. The thermal conductivities $\mathrm{k}_{\alpha}$ and $\mathrm{k}_{\beta}$, like the viscous coefficient $\mu_{\alpha}$, are temperature dependent material properties. The parameters $c_{\beta}$, and $(\rho c)_{\beta}$, are the solid specific heat and heat capacity per unit volume respectively.

The gas enthalpy, $\mathrm{h}_{\alpha}=\hat{\mathrm{u}}_{\alpha}+\left(\mathrm{p}_{\alpha} / \rho_{\alpha}\right)$. Although not immediately obvious from Eq. (5a), it can be shown ${ }^{26}$ that the gradient of the enthalpy flow term, $\vec{\nabla} \cdot\left(\rho_{\alpha} \vec{u}_{\alpha} h_{\alpha}\right)=\vec{\nabla} \cdot\left(\rho_{\alpha} \vec{u}_{\alpha} \hat{u}_{\alpha}\right)+p_{\alpha} \vec{\nabla} \cdot \vec{u}_{\alpha}-\Phi_{\alpha} \quad$ where the viscous 
dissipation function $\Phi_{\alpha}$ is defined as:

$$
\Phi_{\alpha}=-(2 / 3) \mu(\nabla \cdot \overrightarrow{\mathrm{u}})^{2}+2 \mu\left[\left(\frac{\partial \mathrm{u}}{\partial \mathrm{x}}\right)^{2}+\left(\frac{\partial \mathrm{v}}{\partial \mathrm{y}}\right)^{2}+\left(\frac{\partial \mathrm{w}}{\partial \mathrm{z}}\right)^{2}\right]+\mu\left[\left(\frac{\partial \mathrm{v}}{\partial \mathrm{x}}+\frac{\partial \mathrm{u}}{\partial \mathrm{y}}\right)^{2}+\left(\frac{\partial \mathrm{w}}{\partial \mathrm{y}}+\frac{\partial \mathrm{v}}{\partial \mathrm{z}}\right)^{2}+\left(\frac{\partial \mathrm{u}}{\partial \mathrm{z}}+\frac{\partial \mathrm{w}}{\partial \mathrm{x}}\right)^{2}\right]
$$

\section{Equations of State}

Thus far, the system of six equations (mass, three components of momentum and the two phases of energy) containing eight unknowns $\left(\rho_{\alpha}\right.$, three components of $\overrightarrow{\mathrm{u}}_{\alpha}, \mathrm{p}_{\alpha}, \mathrm{T}_{\alpha}, \mathrm{T}_{\beta}, \hat{\mathrm{u}}_{\alpha}$ ) requires two additional equations to close the problem. These equations are the thermodynamic equations of state which can be expressed generally as

$$
\begin{aligned}
& \mathrm{p}_{\alpha}=f_{1}\left(\rho_{\alpha}, \hat{\mathrm{u}}_{\alpha}\right) \\
& \mathrm{T}_{\alpha}=f_{2}\left(\rho_{\alpha}, \hat{\mathrm{u}}_{\alpha}\right)
\end{aligned}
$$

The particular equations of state are arbitrary, in the sense that the particular forms do not affect the form of the governing equations. Ideal or real gas equations can be used.

\section{Entropy Generation Equation}

The differential statement of the second law of thermodynamics ${ }^{3}$ can be re-written in different forms for the $\alpha$ and $\beta$ phases as:

$$
\begin{aligned}
& \alpha \text {-phase } \\
& \mathrm{s}_{\mathrm{gen}, \alpha}^{\prime \prime \prime}=\frac{\partial\left(\rho_{\alpha} \mathrm{s}_{\alpha}\right)}{\partial \mathrm{t}}+\vec{\nabla} \cdot\left(\frac{\overrightarrow{\mathrm{q}}_{\alpha}}{\mathrm{T}_{\alpha}}\right)+\vec{\nabla} \cdot\left(\rho_{\alpha} \mathrm{s}_{\alpha} \overrightarrow{\mathrm{u}}_{\alpha}\right) \geq 0 \\
& \mathrm{~s}_{\mathrm{gen}, \alpha}^{\prime \prime \prime}=-\frac{\overrightarrow{\mathrm{q}}_{\alpha} \cdot \vec{\nabla} \mathrm{T}_{\alpha}}{\mathrm{T}_{\alpha}{ }^{2}}+\frac{\Phi_{\alpha}}{\mathrm{T}_{\alpha}} \geq 0
\end{aligned}
$$

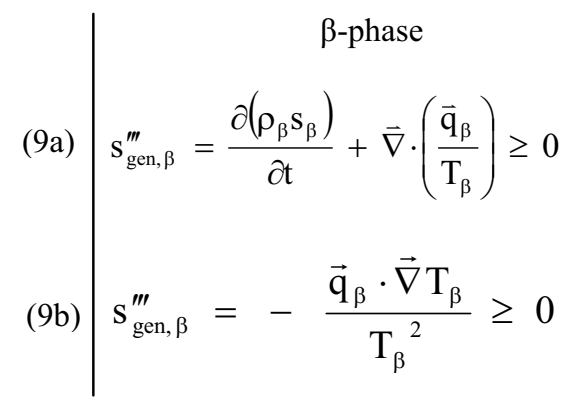

The entropy generation rate for the non-porous regions of the 3-space domain is thus:

$$
\mathrm{s}_{\text {gen, sys(non-porous) }}^{\prime \prime \prime}=\mathrm{s}_{\alpha, \text { gen }}^{\prime \prime \prime}+\mathrm{s}_{\beta, \text { gen }}^{\prime \prime \prime} \geq 0
$$

The second law postulates the existence of entropy, S, a non-conserved thermodynamic property of state that can be created via a generation or production term, $\mathrm{s}_{\text {gen }}^{\prime \prime \prime}$. The parameter $\overrightarrow{\mathrm{q}}$ represents the heat flux vector. The inequality indicates that the entropy generation is always positive except for totally reversible processes, in which case, it is zero. Equations (9) and (10) clearly show that entropy generation is due to system irreversibilities resulting from heat transfer, mass flow and viscous friction.

The foregoing equations represent the generalized, non-volume averaged, microscale equations for a single fluid phase flowing and interacting with a stationary solid phase. 


\section{B. Volume-Averaged Equations}

The flow geometry in the porous regenerator is too complicated to allow for a direct application of the local or microscopic equations. The regenerator presents the need for volume-averaging ${ }^{38}$, an analytical tool for describing the phenomena of compressible flow and heat transfer in a porous medium. The local or microscopic conservation equations for the solid and fluid phases are volume averaged over a representative elementary volume (see Fig.7 below) to arrive at the corresponding "macroscopic" transport equations.

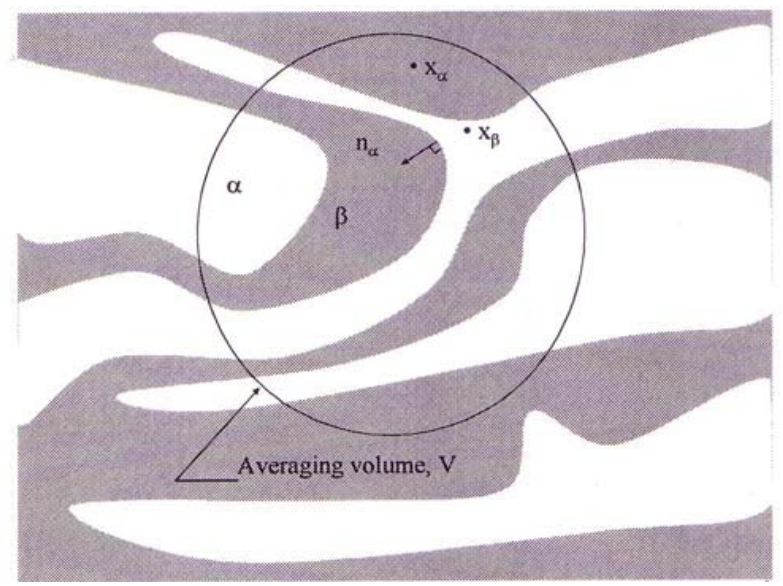

Figure 7. A two-phase model of a porous medium.

The $\alpha$-phase is a Newtonian fluid and the $\beta$-base is the solid part of the porous matrix ${ }^{18}$.

The parameter $\mathrm{n}_{\alpha}$ is the unit normal vector on the $\alpha-\beta$ interface pointing into the $\beta$-phase and $\mathrm{x}$ is the position vector. The subscript on the position vector indicates the phase at that position. The porous matrix is assumed to be stationary and rigid with known thermal properties which are functions of temperature.

The details of the volume averaging method are discussed in Ref.18. The resulting simplified equations are shown below:

\section{Volume-Averaged Conservation of Mass Equation}

$$
\frac{\partial}{\partial \mathrm{t}}\left\langle\rho_{\alpha}\right\rangle^{\alpha}+\nabla \cdot\left(\left\langle\rho_{\alpha}\right\rangle^{\alpha}\left\langle\overrightarrow{\mathrm{u}}_{\alpha}\right\rangle^{\alpha}\right)=0
$$

Note that this simplified macroscopic conserved equation of mass is similar in form to the corresponding microscopic equation (Eq.(3)).

2. Volume-Averaged Conservation of Momentum Equations

$$
\begin{aligned}
& \frac{\partial}{\partial \mathrm{t}}\left(\left\langle\rho_{\alpha}\right\rangle^{\alpha}\left\langle\overrightarrow{\mathrm{u}}_{\alpha}\right\rangle^{\alpha}\right)+\nabla \cdot\left(\left\langle\rho_{\alpha}\right\rangle^{\alpha}\left\langle\overrightarrow{\mathrm{u}}_{\alpha}\right\rangle^{\alpha}\left\langle\overrightarrow{\mathrm{u}}_{\alpha}\right\rangle^{\alpha}\right)+\nabla\left\langle\mathrm{p}_{\alpha}\right\rangle^{\alpha}+\frac{1}{\mathrm{~V}_{\alpha}} \int_{\mathrm{A}_{\alpha \beta}} \mathrm{n}_{\alpha} \cdot\left[\widetilde{\mathrm{I}}\left(\hat{\mathrm{p}}_{\alpha}+\mathrm{n}_{\alpha} \frac{\mu_{\alpha}}{3} \nabla \cdot \hat{\overline{\mathrm{u}}}_{\alpha}\right)+\mu_{\alpha} \nabla \hat{\overline{\mathrm{u}}}_{\alpha}\right] \mathrm{d} \mathrm{S} \\
& +\varepsilon_{\alpha}\left[2\left(\vec{\nabla}\left\langle\mu_{\alpha}\right\rangle^{\alpha} \cdot \vec{\nabla}\right)\left\langle\overrightarrow{\mathrm{u}}_{\alpha}\right\rangle^{\alpha}+\left(\vec{\nabla}\left\langle\mu_{\alpha}\right\rangle^{\alpha}\right) \mathrm{x}\left\langle\vec{\Omega}_{\alpha}\right\rangle^{\alpha}+\frac{2}{3}\left(\vec{\nabla}\left\langle\mu_{\alpha}\right\rangle^{\alpha}\right)^{2}\right]=0
\end{aligned}
$$

The parameter $\varepsilon_{\alpha}=\mathrm{V}_{\alpha} / \mathrm{V}$ is the gas phase volume fraction, or porosity. $\mathrm{V}_{\alpha}$ is the gas phase volume and $\mathrm{V}$ is the arbitrary averaging volume containing the porous medium (see Figure 7 above). The notations \langle\rangle and ${ }^{\wedge}$ indicate a volume average and a spatial deviation quantity respectively. 


\section{Volume-Averaged Conservation of Energy Equation}

$$
\begin{array}{c|c}
\alpha \text {-phase } & \beta \text {-phase } \\
\frac{\partial}{\partial \mathrm{t}}\left(\left\langle\rho_{\alpha}\right\rangle^{\alpha}\left\langle\hat{\mathrm{u}}_{\alpha}\right\rangle^{\alpha}\right)+\nabla \cdot\left(\left\langle\rho_{\alpha}\right\rangle^{\alpha}\left\langle\overrightarrow{\mathrm{u}}_{\alpha}\right\rangle^{\alpha}\left\langle\mathrm{h}_{\alpha}\right\rangle^{\alpha}-\right. & (\rho \mathrm{c})_{\beta} \frac{\partial\left\langle\mathrm{T}_{\beta}\right\rangle^{\beta}}{\partial \mathrm{t}}-\nabla \cdot\left[\mathrm{k}_{\beta} \tau_{\beta} \nabla\left\langle\mathrm{T}_{\beta}\right\rangle^{\beta}\right] \\
\left.\mathrm{N}_{\mathrm{k}} \mathrm{k}_{\alpha} \nabla\left\langle\mathrm{T}_{\alpha}\right\rangle^{\alpha}\right)-\alpha_{v} \mathrm{H}\left(\left\langle\mathrm{T}_{\beta}\right\rangle^{\beta}-\left\langle\mathrm{T}_{\alpha}\right\rangle^{\alpha}\right)=0 & +\frac{\varepsilon_{\alpha}}{\varepsilon_{\beta}} \alpha_{v} \mathrm{H}\left(\left\langle\mathrm{T}_{\beta}\right\rangle^{\beta}-\left\langle\mathrm{T}_{\alpha}\right\rangle^{\alpha}\right)=0
\end{array}
$$

Volume averaging in this case results in the film heat transfer term, a term additional to the standard form of the $\alpha$ and $\beta$-phase microscopic energy equations (5a, $5 b$ ). Gedeon ${ }^{14}$ recognized $N_{k}$ as axial conductivity enhancement due to thermal dispersion having a Peclet dependence of the form $\left(c_{1}+c_{2} \operatorname{Re}^{m} \operatorname{Pr}^{\mathrm{n}}\right)$ where $\mathrm{c}_{1}$ and $\mathrm{c}_{2}$ are constants and $\mathrm{m}$ and $\mathrm{n}$ are positive constants. This functional form for the dispersion agrees with other empirical predictions $\mathrm{s}^{32,34}$. The parameters $\alpha_{v}$ and $H$ are the surface area per unit volume and the convective heat transfer coefficient respectively.

\section{Volume-Averaged Entropy Generation Equation}

$$
\begin{aligned}
& \left\langle\mathrm{s}_{\alpha, \text { gen }}^{\prime \prime \prime}\right\rangle^{\alpha}=\frac{\partial\left(\left\langle\rho_{\alpha}\right\rangle^{\alpha}\left\langle\mathrm{s}_{\alpha}\right\rangle^{\alpha}\right)}{\partial \mathrm{t}}+\nabla \cdot\left(\left\langle\rho_{\alpha}\right\rangle^{\alpha}\left\langle\mathrm{s}_{\alpha}\right\rangle^{\alpha}\left\langle\overrightarrow{\mathrm{u}}_{\alpha}\right\rangle^{\alpha}\right) \mid\left\langle\mathrm{s}_{\beta, \text { gen }}^{\prime \prime \prime}\right\rangle^{\beta}=\rho_{\beta} \frac{\partial\left\langle\mathrm{s}_{\beta}\right\rangle^{\beta}}{\partial \mathrm{t}}-\frac{1}{\varepsilon_{\beta}} \nabla \cdot\left[\mathrm{k}_{\beta}\left\langle\frac{\nabla \mathrm{T}}{\mathrm{T}_{\beta}}\right\rangle^{\beta}\right] \\
& -\nabla \cdot\left[\frac{\mathrm{N}_{\mathrm{k}} \mathrm{k}_{\alpha}}{\left\langle\mathrm{T}_{\alpha}\right\rangle^{\alpha}} \nabla\left\langle\mathrm{T}_{\alpha}\right\rangle^{\alpha}\right]-\frac{\alpha_{v} \mathrm{H}}{\left\langle\mathrm{T}_{\beta}\right\rangle^{\beta}}\left\langle\left\langle\mathrm{T}_{\beta}\right\rangle^{\beta}-\left\langle\mathrm{T}_{\alpha}\right\rangle^{\alpha}\right) \\
& +\frac{\varepsilon_{\alpha} \alpha_{v} \mathrm{H}}{\varepsilon_{\beta}\left\langle\mathrm{T}_{\alpha}\right\rangle^{\alpha}}\left(\left\langle\mathrm{T}_{\beta}\right\rangle^{\beta}-\left\langle\mathrm{T}_{\alpha}\right\rangle^{\alpha}\right)
\end{aligned}
$$

After considerable simplification using the Maxwell relations for a pure substance ${ }^{3}$, momentum and energy equations the entropy generation equation reduces to ${ }^{18}$ :

$$
\begin{aligned}
& \begin{array}{c}
\alpha \text {-phase } \\
\left\langle\mathrm{s}_{\alpha, \text { gen }}^{\prime \prime \prime}\right\rangle^{\alpha}=\mathrm{N}_{\mathrm{k}} \mathrm{k}_{\alpha}\left(\frac{\nabla\left\langle\mathrm{T}_{\alpha}\right\rangle^{\alpha}}{\left\langle\mathrm{T}_{\alpha}\right\rangle^{\alpha}}\right)^{2}+\frac{\alpha_{v} \mathrm{H}}{\left\langle\mathrm{T}_{\alpha}\right\rangle^{\alpha}\left\langle\mathrm{T}_{\beta}\right\rangle^{\beta}}\left|\left\langle\mathrm{T}_{\beta}\right\rangle^{\beta}-\left\langle\mathrm{T}_{\alpha}\right\rangle^{\alpha}\right|^{2}
\end{array} \\
& -\frac{\left\langle\overrightarrow{\mathrm{u}}_{\alpha}\right\rangle^{\alpha}}{\left\langle\mathrm{T}_{\alpha}\right\rangle^{\alpha}} \nabla\left\langle\mathrm{p}_{\alpha}\right\rangle^{\alpha} \\
& \left\langle\mathrm{s}_{\beta, \text { gen }}^{\prime \prime \prime}\right\rangle^{\beta}=\mathrm{k}_{\beta}\left(\frac{\nabla\left\langle\mathrm{T}_{\beta}\right\rangle^{\beta}}{\left\langle\mathrm{T}_{\beta}\right\rangle^{\beta}}\right)^{2} \\
& +\frac{\varepsilon_{\alpha} \alpha_{v} \mathrm{H}}{\varepsilon_{\beta}\left\langle\mathrm{T}_{\alpha}\right\rangle^{\alpha}\left\langle\mathrm{T}_{\beta}\right\rangle^{\beta}}\left|\left\langle\mathrm{T}_{\beta}\right\rangle^{\beta}-\left\langle\mathrm{T}_{\alpha}\right\rangle^{\alpha}\right|^{2}
\end{aligned}
$$

Thus the entropy generation rate equation for the gas-matrix or porous system can be written as

$$
\left\langle\mathrm{s}_{\text {gen,sys (porous) }}^{\prime \prime \prime}\right\rangle=\varepsilon_{\alpha}\left\langle\mathrm{s}_{\alpha, \text { gen }}^{\prime \prime \prime}\right\rangle+\varepsilon_{\beta}\left\langle\mathrm{s}_{\beta, \text { gen }}^{\prime \prime \prime}\right\rangle \geq 0
$$

Substituting Eqs. (15a and 15b) into Eq. (16) the entropy generation equation for the porous regenerator system can be written as

$$
\begin{aligned}
&\left\langle\mathrm{s}_{\text {gen, sys }}^{\prime \prime \prime}\right\rangle= \varepsilon_{\alpha} \mathrm{N}_{\mathrm{k}} \mathrm{k}_{\alpha}\left(\frac{\nabla\left\langle\mathrm{T}_{\alpha}\right\rangle^{\alpha}}{\left\langle\mathrm{T}_{\alpha}\right\rangle^{\alpha}}\right)^{2}+\varepsilon_{\beta} \mathrm{k}_{\beta}\left(\frac{\nabla\left\langle\mathrm{T}_{\beta}\right\rangle^{\beta}}{\left\langle\mathrm{T}_{\beta}\right\rangle^{\beta}}\right)^{2}+\frac{\varepsilon_{\alpha} \alpha_{\mathrm{v}} \mathrm{H}}{\left\langle\mathrm{T}_{\alpha}\right\rangle^{\alpha}\left\langle\mathrm{T}_{\beta}\right\rangle^{\beta}}\left\langle\mathrm{T}_{\beta}\right\rangle^{\beta}-\left.\left\langle\mathrm{T}_{\alpha}\right\rangle^{\alpha}\right|^{2}-\varepsilon_{\alpha} \frac{\left\langle\overrightarrow{\mathrm{u}}_{\alpha}\right\rangle^{\alpha}}{\left\langle\mathrm{T}_{\alpha}\right\rangle^{\alpha}} \nabla\left\langle\mathrm{p}_{\alpha}\right\rangle^{\alpha} \geq 0 \\
& \text { Gas conduction Matrix conduction } \quad \text { Film heat trans fer viscous and inertial } \\
& \text { losses }
\end{aligned}
$$

Eq. (17) indicates that the sources of irreversibility are gas conduction, matrix conduction, film heat transfer and viscous and inertial losses. 
The volume-averaging technique assumes that the velocity, pressure and temperature can be represented in terms of a single large-scale averaged quantity in regions having significantly different mechanical and thermal properties. This assumption provides the basis for the porous-media models available in two CFD codes, Fluent and CFD-ACE, used at GRC for modeling Infinia's Stirling TDC. The standard models assume that the porous matrix and the fluid are in local thermal equilibrium at each spatial interface between them. This is believed to be a poor assumption for the oscillating-flow environment within Stirling regenerators ${ }^{29,34}$. 1-D regenerator models, used in Stirling engine design, use thermal non-equilibrium models and suggest regenerator porous matrix and gas average temperatures can differ by several degrees at a given axial location and time during the cycle ${ }^{33}$. A survey of the porous-media literature supports the need for thermal non-equilibrium porous-media models for thermal storage applications such as Stirling regenerators ${ }^{1,2,22,23,30}$. It is anticipated that a thermal non-equilibrium model such as that presented here, when incorporated in commercial CFD codes, will improve our ability to accurately model Stirling regenerators relative to current thermal-equilibrium porous-media models.

\section{Second Law Analysis via Entropy Generation}

Equations (10) and (17) contain several source terms which represent thermal dispersion, gas-to-matrix heat transfer and viscous and inertial friction. These fundamental mechanisms which are associated with internal system irreversibilities generate entropy within thermo-fluid systems. The second law analysis via entropy generation is concerned mainly with the computation of the available energy losses due to these system irreversibilities.

Entropy generation may be calculated in one of two ways: by entropy transfer accompanied by heat transfer and mass flow across the external boundaries of a system (external entropy generation) or by entropy generated by internal processes due to heat transfer and viscous and inertial losses (internal entropy generation). In principle, the two methods of accounting should give the same answer. Discrepancies which usually arise are often attributable to numerical errors (finite-difference truncation errors, round-off errors, interpolation errors, etc.).

\section{External Entropy Generation}

The external entropy calculation is made by integrating the heat transfer and mass flow over the surface between the internal calculation domain and the environment. In a reciprocating device like the MIT test rig, the external entropy generation over each period is obtained via a cyclic integral.

\subsection{Non-Porous Regions of the 3-space Domain:}

Substituting equations (9a) and (9c) in Eq. (10) gives

$$
\mathrm{s}_{\text {gen, sys (non-porous) }}^{\prime \prime \prime}=\left\{\frac{\partial\left(\rho_{\alpha} \mathrm{s}_{\alpha}\right)}{\partial \mathrm{t}}+\vec{\nabla} \cdot\left(\frac{\overrightarrow{\mathrm{q}}_{\alpha}}{\mathrm{T}_{\alpha}}\right)+\vec{\nabla} \cdot\left(\rho_{\alpha} \overrightarrow{\mathrm{u}}_{\alpha} \mathrm{s}_{\alpha}\right)\right\}+\left\{\frac{\partial\left(\rho_{\beta} \mathrm{s}_{\beta}\right)}{\partial \mathrm{t}}+\vec{\nabla} \cdot\left(\frac{\overrightarrow{\mathrm{q}}_{\beta}}{\mathrm{T}_{\beta}}\right)\right\} \geq 0
$$

Expressing Eq. (18) in integral form we get

$$
\begin{aligned}
& \int_{c v} s_{\substack{\text { gen, sys } \\
\text { (non-porous) }}}^{\prime \prime \prime} d V=\left\{\int_{d v} \frac{\partial\left(\rho_{\alpha} s_{\alpha}\right)}{\partial t} d V+\int_{d s} \frac{\overrightarrow{\mathrm{q}}_{\alpha}}{\mathrm{T}_{\alpha}} \cdot \hat{\mathrm{n}}_{\alpha} \mathrm{dA}+\int_{\mathrm{ss}} \rho_{\alpha} \overrightarrow{\mathrm{u}}_{\alpha} \mathrm{s}_{\alpha} \cdot \hat{\mathrm{n}}_{\alpha} \mathrm{dA}\right\}+\left\{\int_{\mathrm{cv}} \frac{\partial\left(\rho_{\beta} \mathrm{s}_{\beta}\right)}{\partial \mathrm{t}} \mathrm{dV}+\int_{\mathrm{s}} \frac{\overrightarrow{\mathrm{q}}_{\beta}}{\mathrm{T}_{\beta}} \cdot \hat{\mathrm{n}}_{\beta} \mathrm{dA}\right\} \geq 0 \\
& \dot{\mathrm{S}}_{\text {gen, sys (non-porous) }}=\int_{\mathrm{vv}}\left[\frac{\partial\left(\rho_{\alpha} \mathrm{s}_{\alpha}\right)}{\partial \mathrm{t}}+\frac{\partial\left(\rho_{\beta} \mathrm{s}_{\beta}\right)}{\partial \mathrm{t}}\right] \mathrm{dV}+\int_{\mathrm{ds}}\left[\left(\frac{\overrightarrow{\mathrm{q}}_{\alpha}}{\mathrm{T}_{\alpha}} \cdot \hat{\mathrm{n}}_{\alpha}+\frac{\overrightarrow{\mathrm{q}}_{\beta}}{\mathrm{T}_{\beta}} \cdot \hat{\mathrm{n}}_{\beta}\right)+\rho_{\alpha} \overrightarrow{\mathrm{u}}_{\alpha} \mathrm{s}_{\alpha} \cdot \hat{\mathrm{n}}_{\alpha}\right] \mathrm{dA} \geq 0
\end{aligned}
$$

Taking the cyclic integral of Eq. (18) over one period gives:

$$
\begin{aligned}
& \oint_{\text {period }} \dot{\mathrm{S}}_{\substack{\text { gen, sys } \\
\text { (non -porous) }}} \mathrm{dt}=\oint_{\text {period }}\left\{\int_{\mathrm{dv}}\left[\frac{\partial\left(\rho_{\alpha} \mathrm{s}_{\alpha}\right)}{\partial \mathrm{t}}+\frac{\partial\left(\rho_{\beta} \mathrm{s}_{\beta}\right)}{\partial \mathrm{t}}\right] \mathrm{dV}+\int_{\mathrm{s}}\left[\left(\frac{\overrightarrow{\mathrm{q}}_{\alpha}}{\mathrm{T}_{\alpha}} \cdot \hat{\mathrm{n}}_{\alpha}+\frac{\overrightarrow{\mathrm{q}}_{\beta}}{\mathrm{T}_{\beta}} \cdot \hat{\mathrm{n}}_{\beta}\right)+\rho_{\alpha} \overrightarrow{\mathrm{u}}_{\alpha} \mathrm{s}_{\alpha} \cdot \hat{\mathrm{n}}_{\alpha}\right] \mathrm{dA}\right\} \mathrm{dt} \geq 0 \\
& \dot{\mathrm{S}}_{\substack{\text { gen, sys, cyl(ext) } \\
\text { (non-porous) }}}=\oint_{\text {eriod }} \int_{d \mathrm{~s}}\left(\frac{\overrightarrow{\mathrm{q}}_{\alpha}}{\mathrm{T}_{\alpha}} \cdot \hat{\mathrm{n}}_{\alpha}+\frac{\overrightarrow{\mathrm{q}}_{\beta}}{\mathrm{T}_{\beta}} \cdot \hat{\mathrm{n}}_{\beta}\right) \mathrm{dAdt}+\oint_{\text {eriod }} \int_{\alpha \mathrm{s}} \rho_{\alpha} \overrightarrow{\mathrm{u}}_{\alpha} \mathrm{s} \mathrm{s}_{\alpha} \cdot \hat{\mathrm{n}}_{\alpha} \mathrm{dAdt} \geq 0
\end{aligned}
$$


The integral terms account for the net entropy transfer with heat and mass out of the non-porous regions of the 3space domain into the surrounding environment during each cycle of operation.

\subsection{Porous Regions of the 3-space Domain:}

Substituting equations (14a and 14b) into Eq. (16) gives:

$$
\begin{array}{r}
\left\langle\mathrm{s}_{\text {gen,sys }}^{\prime \prime \prime}\right\rangle=\varepsilon_{\alpha}\left\{\frac{\partial\left(\left\langle\rho_{\alpha}\right\rangle^{\alpha}\left\langle\mathrm{s}_{\alpha}\right\rangle^{\alpha}\right)}{\partial \mathrm{t}}+\nabla \cdot\left(\left\langle\rho_{\alpha}\right\rangle^{\alpha}\left\langle\mathrm{s}_{\alpha}\right\rangle^{\alpha}\left\langle\overrightarrow{\mathrm{u}}_{\alpha}\right\rangle^{\alpha}\right)-\nabla \cdot\left[\frac{\mathrm{N}_{\mathrm{k}} \mathrm{k}_{\alpha}}{\left\langle\mathrm{T}_{\alpha}\right\rangle^{\alpha}} \nabla\left\langle\mathrm{T}_{\alpha}\right\rangle^{\alpha}\right]-\frac{\alpha_{v} \mathrm{H}}{\left\langle\mathrm{T}_{\beta}\right\rangle^{\beta}}\left\langle\left\langle\mathrm{T}_{\beta}\right\rangle^{\beta}-\left\langle\mathrm{T}_{\alpha}\right\rangle^{\alpha}\right)\right\}+ \\
\varepsilon_{\beta}\left\{\rho_{\beta} \frac{\partial\left\langle\mathrm{s}_{\beta}\right\rangle^{\beta}}{\partial \mathrm{t}}-\frac{1}{\varepsilon_{\beta}} \nabla \cdot\left[\mathrm{k}_{\beta}\left\langle\frac{\nabla \mathrm{T}}{\mathrm{T}_{\beta}}\right\rangle^{\beta}\right]+\frac{\varepsilon_{\alpha} \alpha_{v} \mathrm{H}}{\varepsilon_{\beta}\left\langle\mathrm{T}_{\alpha}\right\rangle^{\alpha}}\left\langle\left\langle\mathrm{T}_{\beta}\right\rangle^{\beta}-\left\langle\mathrm{T}_{\alpha}\right\rangle^{\alpha}\right)\right\} \geq 0
\end{array}
$$

Expressing Eq. (23) in integral form we get:

$$
\begin{aligned}
& \int_{\mathrm{vv}}\left\langle\mathrm{s}_{\mathrm{gen, \text {sys }}}^{\prime \prime \prime} \mathrm{dV}=\int_{\mathrm{dv}} \varepsilon_{\alpha}\left\{\frac{\partial\left(\left\langle\rho_{\alpha}\right\rangle^{\alpha}\left\langle\mathrm{s}_{\alpha}\right\rangle^{\alpha}\right)}{\partial \mathrm{t}}+\nabla \cdot\left(\left\langle\rho_{\alpha}\right\rangle^{\alpha}\left\langle\mathrm{s}_{\alpha}\right\rangle^{\alpha}\left\langle\overrightarrow{\mathrm{u}}_{\alpha}\right\rangle^{\alpha}\right)-\nabla \cdot\left[\frac{\mathrm{N}_{\mathrm{k}} \mathrm{k}_{\alpha}}{\left\langle\mathrm{T}_{\alpha}\right\rangle^{\alpha}} \nabla\left\langle\mathrm{T}_{\alpha}\right\rangle^{\alpha}\right]-\frac{\alpha_{v} \mathrm{H}}{\left\langle\mathrm{T}_{\beta}\right\rangle^{\beta}}\left\langle\left\langle\mathrm{T}_{\beta}\right\rangle^{\beta}-\left\langle\mathrm{T}_{\alpha}\right\rangle^{\alpha}\right)\right\} \mathrm{dV}+\right. \\
& \int_{\mathrm{cv}} \varepsilon_{\beta}\left\{\rho_{\beta} \frac{\partial\left\langle\mathrm{s}_{\beta}\right\rangle^{\beta}}{\partial \mathrm{t}}-\frac{1}{\varepsilon_{\beta}} \nabla \cdot\left[\mathrm{k}_{\beta}\left\langle\frac{\nabla \mathrm{T}}{\mathrm{T}_{\beta}}\right\rangle^{\beta}\right]+\frac{\varepsilon_{\alpha} \alpha_{v} \mathrm{H}}{\varepsilon_{\beta}\left\langle\mathrm{T}_{\alpha}\right\rangle^{\alpha}}\left\langle\left\langle\mathrm{T}_{\beta}\right\rangle^{\beta}-\left\langle\mathrm{T}_{\alpha}\right\rangle^{\alpha}\right)\right\} \mathrm{dV} \quad \geq 0 \\
& \left\langle\dot{\mathrm{S}}_{\substack{\text { gen,sys } \\
\text { (porous) }}}\right\rangle=\int_{\mathrm{vv}}\left[\varepsilon_{\alpha} \frac{\partial\left(\left\langle\rho_{\alpha}\right\rangle^{\alpha}\left\langle\mathrm{s}_{\alpha}\right\rangle^{\alpha}\right)}{\partial \mathrm{t}}+\varepsilon_{\beta} \rho_{\beta} \frac{\partial\left\langle\mathrm{s}_{\beta}\right\rangle^{\beta}}{\partial \mathrm{t}}\right] \mathrm{dV}+\int_{\mathrm{s}} \varepsilon_{\alpha}\left(\left\langle\rho_{\alpha}\right\rangle^{\alpha}\left\langle\mathrm{s}_{\alpha}\right\rangle^{\alpha}\left\langle\overrightarrow{\mathrm{u}}_{\alpha}\right\rangle^{\alpha}\right) \cdot \hat{\mathrm{n}}_{\alpha} \mathrm{dA}-\int_{\mathrm{s}}\left[\frac{\varepsilon_{\alpha} \mathrm{N}_{\mathrm{k}} \mathrm{k}_{\alpha}}{\left\langle\mathrm{T}_{\alpha}\right\rangle^{\alpha}} \nabla\left\langle\mathrm{T}_{\alpha}\right\rangle^{\alpha}\right] \cdot \hat{\mathrm{n}}_{\alpha} \mathrm{dA} \\
& -\int_{d s}\left[\mathrm{k}_{\beta}\left\langle\frac{\nabla \mathrm{T}_{\beta}}{\mathrm{T}_{\beta}}\right\rangle^{\beta}\right] \cdot \hat{\mathrm{n}}_{\beta} \mathrm{dA} \geq 0
\end{aligned}
$$

Taking the cyclic integral of Eq. (25) over one period gives:

$$
\begin{aligned}
& \oint_{\text {period }} \dot{\mathrm{S}}_{\substack{\text { gen, sys } \\
\text { porous }}} \mathrm{dt}=\oint_{\text {period }}\left\{\int_{\mathrm{cv}}\left[\varepsilon_{\alpha} \frac{\partial\left(\left\langle\rho_{\alpha}\right\rangle^{\alpha}\left\langle\mathrm{s}_{\alpha}\right\rangle^{\alpha}\right)}{\partial \mathrm{t}}+\varepsilon_{\beta} \rho_{\beta} \frac{\partial\left\langle\mathrm{s}_{\beta}\right\rangle^{\beta}}{\partial \mathrm{t}}\right] \mathrm{dV}+\int_{\mathrm{cs}} \varepsilon_{\alpha}\left(\left\langle\rho_{\alpha}\right\rangle^{\alpha}\left\langle\mathrm{s}_{\alpha}\right\rangle^{\alpha}\left\langle\overrightarrow{\mathrm{u}}_{\alpha}\right\rangle^{\alpha}\right) \cdot \hat{\mathrm{n}}_{\alpha} \mathrm{dA}\right. \\
& \left.-\int_{d s}\left[\frac{\varepsilon_{\alpha} \mathrm{N}_{\mathrm{k}} \mathrm{k}_{\alpha}}{\left\langle\mathrm{T}_{\alpha}\right\rangle^{\alpha}} \nabla\left\langle\mathrm{T}_{\alpha}\right\rangle^{\alpha}\right] \cdot \hat{\mathrm{n}}_{\alpha} \mathrm{dA}-\int_{\mathrm{cs}}\left[\mathrm{k}_{\beta}\left\langle\frac{\nabla \mathrm{T}_{\beta}}{\mathrm{T}_{\beta}}\right\rangle^{\beta}\right] \cdot \hat{\mathrm{n}}_{\beta} \mathrm{dA}\right\} \mathrm{dt} \geq 0 \\
& \left\langle\dot{\mathrm{S}}_{\substack{\text { gen,sys, cyl (ext) } \\
\text { porous }}}\right\rangle=\oint_{\text {period }} \int_{-s}\left\{\varepsilon_{\alpha}\left(\left\langle\rho_{\alpha}\right\rangle^{\alpha}\left\langle\mathrm{s}_{\alpha}\right\rangle^{\alpha}\left\langle\overrightarrow{\mathrm{u}}_{\alpha}\right\rangle^{\alpha}\right) \cdot \hat{\mathrm{n}}_{\alpha}-\left[\frac{\varepsilon_{\alpha} \mathrm{N}_{\mathrm{k}} \mathrm{k}_{\alpha}}{\left\langle\mathrm{T}_{\alpha}\right\rangle^{\alpha}} \nabla\left\langle\mathrm{T}_{\alpha}\right\rangle^{\alpha}\right] \cdot \hat{\mathrm{n}}_{\alpha}-\left[\mathrm{k}_{\beta}\left\langle\frac{\nabla \mathrm{T}_{\beta}}{\mathrm{T}_{\beta}}\right\rangle^{\beta}\right] \cdot \hat{\mathrm{n}}_{\beta}\right\} \mathrm{dAdt} \geq 0 \\
& \left\langle\dot{\mathrm{S}}_{\substack{\text { gen,sys, cyl(ext) } \\
\text { porous }}}\right\rangle=\oint_{\text {eriod }} \int_{d s}\left(\varepsilon_{\alpha} \mathrm{N}_{\mathrm{k}} \frac{\left\langle\overrightarrow{\mathrm{q}}_{\alpha}\right\rangle^{\alpha}}{\left\langle\mathrm{T}_{\alpha}\right\rangle^{\alpha}} \cdot \hat{\mathrm{n}}_{\alpha}+\frac{\left\langle\overrightarrow{\mathrm{q}}_{\beta}\right\rangle^{\beta}}{\left\langle\mathrm{T}_{\beta}\right\rangle^{\beta}} \cdot \hat{\mathrm{n}}_{\beta}\right) \mathrm{dAdt}+\oint_{\text {period }} \int_{\mathrm{ss}} \varepsilon_{\alpha}\left(\left\langle\rho_{\alpha}\right\rangle^{\alpha}\left\langle\mathrm{s}_{\alpha}\right\rangle^{\alpha}\left\langle\overrightarrow{\mathrm{u}}_{\alpha}\right\rangle^{\alpha}\right) \cdot \hat{\mathrm{n}}_{\alpha} \mathrm{dAdt} \geq 0
\end{aligned}
$$

The integral terms account for the net entropy transfer with heat and mass out of the porous regions of the 3-space domain into the surrounding environment during each cycle of operation. 


\section{Internal Entropy Generation}

Internal entropy generation can be accounted for by tallying up the individual entropy generations due to, heat transfer, viscous and inertial losses in all internal processes.

\subsection{Non-Porous Regions of the 3-space Domain:}

Substituting equations (9b) and (9d) in Eq. (10) gives

$$
\mathrm{s}_{\text {gen, sys }}^{\prime \prime \prime}=-\frac{\overrightarrow{\mathrm{q}}_{\alpha} \cdot \vec{\nabla} \mathrm{T}_{\alpha}}{\mathrm{T}_{\alpha}{ }^{2}}+\frac{\Phi_{\alpha}}{\mathrm{T}_{\alpha}}-\frac{\overrightarrow{\mathrm{q}}_{\beta} \cdot \vec{\nabla} \mathrm{T}_{\beta}}{\mathrm{T}_{\beta}{ }^{2}} \geq 0
$$

Expressing Eq.(29) in integral form we get

$$
\begin{aligned}
& \int_{\mathrm{cv}} \mathrm{s}_{\substack{\text { gen, sys } \\
\text { (non-porous) }}}^{\prime \prime} \mathrm{dV}=-\int_{\mathrm{cv}}\left(\frac{\overrightarrow{\mathrm{q}}_{\alpha} \cdot \vec{\nabla} \mathrm{T}_{\alpha}}{\mathrm{T}_{\alpha}{ }^{2}}+\frac{\overrightarrow{\mathrm{q}}_{\beta} \cdot \vec{\nabla} \mathrm{T}_{\beta}}{\mathrm{T}_{\beta}{ }^{2}}\right) \mathrm{dV}+\int_{\mathrm{cv}} \frac{\Phi}{\mathrm{T}_{\alpha}} \mathrm{dV} \geq 0 \\
& \dot{\mathrm{S}}_{\text {gen, sys (non-porous) }}=-\int_{\mathrm{cv}}\left(\frac{\overrightarrow{\mathrm{q}}_{\alpha} \cdot \vec{\nabla} \mathrm{T}_{\alpha}}{\mathrm{T}_{\alpha}{ }^{2}}+\frac{\overrightarrow{\mathrm{q}}_{\beta} \cdot \vec{\nabla} \mathrm{T}_{\beta}}{\mathrm{T}_{\beta}{ }^{2}}\right) \mathrm{dV}+\int_{\mathrm{cv}} \frac{\Phi}{\mathrm{T}_{\alpha}} \mathrm{dV} \geq 0
\end{aligned}
$$

Taking the cyclic integral of Eq. (31) over one period gives:

$$
\dot{\mathrm{S}}_{\text {gen, cyl (int) }}=-\oint_{\text {period }} \int_{\mathrm{cv}}\left(\frac{\overrightarrow{\mathrm{q}}_{\alpha} \cdot \vec{\nabla} \mathrm{T}_{\alpha}}{\mathrm{T}_{\alpha}{ }^{2}}+\frac{\overrightarrow{\mathrm{q}}_{\beta} \cdot \vec{\nabla} \mathrm{T}_{\beta}}{\mathrm{T}_{\beta}{ }^{2}}\right) \mathrm{dV} \mathrm{dt}+\oint_{\text {period }} \int_{\mathrm{cv}} \frac{\Phi_{\alpha}}{\mathrm{T}_{\alpha}} \mathrm{dV} \mathrm{dt} \geq 0
$$

Note that:

$$
\begin{aligned}
\frac{\overrightarrow{\mathrm{q}} \cdot \vec{\nabla} \mathrm{T}}{\mathrm{T}^{2}} & =\frac{\mathrm{k}}{\mathrm{T}^{2}}\left[\left(\frac{\partial \mathrm{T}}{\partial \mathrm{x}}\right)^{2}+\left(\frac{\partial \mathrm{T}}{\partial \mathrm{y}}\right)^{2}+\left(\frac{\partial \mathrm{T}}{\partial \mathrm{z}}\right)^{2}\right] \\
\frac{\Phi}{\mathrm{T}} & =\frac{1}{\mathrm{~T}}\left\{(-2 / 3) \mu(\vec{\nabla} \cdot \overrightarrow{\mathrm{u}})^{2}+2 \mu\left[\left(\frac{\partial \mathrm{u}}{\partial \mathrm{x}}\right)^{2}+\left(\frac{\partial \mathrm{v}}{\partial \mathrm{y}}\right)^{2}+\left(\frac{\partial \mathrm{w}}{\partial \mathrm{z}}\right)^{2}\right]+\mu\left[\left(\frac{\partial \mathrm{v}}{\partial \mathrm{x}}+\frac{\partial \mathrm{u}}{\partial \mathrm{y}}\right)^{2}+\left(\frac{\partial \mathrm{w}}{\partial \mathrm{y}}+\frac{\partial \mathrm{v}}{\partial \mathrm{z}}\right)^{2}+\left(\frac{\partial \mathrm{u}}{\partial \mathrm{z}}+\frac{\partial \mathrm{w}}{\partial \mathrm{x}}\right)^{2}\right]\right\}
\end{aligned}
$$

Eq.(32) accounts for the internal entropy generation in a reciprocating device like the MIT test rig. From Eqs. (33 and 34), we see that the first term on the right-hand side of Eq. (32) which involves the squares of the temperature gradients (see Eq. (33)) is the entropy generation due to conductive heat flow while the second term which involves the squares of the velocity gradients (see Eq. (34)) is the contribution due to viscous dissipation. Equation (32) seems to imply that the internal thermodynamic losses can be minimized by minimizing the magnitude of these local temperature and velocity gradients and the sum of their squares, throughout the domain of interest. Although a reduction in the fluid viscosity would lead to a reduction of the velocity gradients at the wall and the boundary layer viscous losses and an increase the fluid thermal conductivity would reduce the temperature gradients at the wall, and the conductive heat transfer loss; these measures are difficult or impractical to implement. It is however practical to minimize local velocity and temperature gradients via changes in the geometry and operating conditions such as frequency of operation. 


\subsection{Porous Regions of the 3-space Domain:}

Expressing the system entropy generation equation, Eq.(17), in integral form gives

$$
\begin{array}{r}
\int_{\mathrm{dv}}\left\langle\mathrm{s}_{\text {gen, sys }}^{\prime \prime \prime}\right\rangle^{\alpha} \mathrm{dV}=\int_{\mathrm{cv}}\left\{\varepsilon_{\alpha} \mathrm{N}_{\mathrm{k}} \mathrm{k}_{\alpha}\left(\frac{\nabla\left\langle\mathrm{T}_{\alpha}\right\rangle^{\alpha}}{\left\langle\mathrm{T}_{\alpha}\right\rangle^{\alpha}}\right)^{2}+\varepsilon_{\beta} \mathrm{k}_{\beta}\left(\frac{\nabla\left\langle\mathrm{T}_{\beta}\right\rangle^{\beta}}{\left\langle\mathrm{T}_{\beta}\right\rangle^{\beta}}\right)^{2}+\left(\frac{\varepsilon_{\alpha} \alpha_{\mathrm{v}} \mathrm{H}}{\left\langle\mathrm{T}_{\alpha}\right\rangle^{\alpha}\left\langle\mathrm{T}_{\beta}\right\rangle^{\beta}}\left\langle\mathrm{T}_{\beta}\right\rangle^{\beta}-\left.\left\langle\mathrm{T}_{\alpha}\right\rangle^{\alpha}\right|^{2}\right)\right. \\
\left.-\left(\varepsilon_{\alpha} \frac{\left\langle\overrightarrow{\mathrm{u}}_{\alpha}\right\rangle^{\alpha}}{\left\langle\mathrm{T}_{\alpha}\right\rangle^{\alpha}} \nabla\left\langle\mathrm{p}_{\alpha}\right\rangle^{\alpha}\right)\right\} \mathrm{dV} \geq 0
\end{array}
$$

Taking the cyclic integral of Eq.(35) over one period gives:

$$
\begin{aligned}
& \left\langle\dot{\mathrm{S}}_{\text {gen,cyl (int.) }\rangle}\right\rangle=\oint_{\text {eriod }} \int_{\mathrm{vv}}\left\{\varepsilon_{\alpha} \mathrm{N}_{\mathrm{k}} \mathrm{k}_{\alpha}\left(\frac{\nabla\left\langle\mathrm{T}_{\alpha}\right\rangle^{\alpha}}{\left\langle\mathrm{T}_{\alpha}\right\rangle^{\alpha}}\right)^{2}+\varepsilon_{\beta} \mathrm{k}_{\beta}\left(\frac{\nabla\left\langle\mathrm{T}_{\beta}\right\rangle^{\beta}}{\left\langle\mathrm{T}_{\beta}\right\rangle^{\beta}}\right)^{2}\right\} \mathrm{dVdt}+\oint_{\text {eriod }} \int_{\mathrm{vv}}\left(\frac{\varepsilon_{\alpha} \alpha_{v} \mathrm{H}}{\left\langle\mathrm{T}_{\alpha}\right\rangle^{\alpha}\left\langle\mathrm{T}_{\beta}\right\rangle^{\beta}}\left\langle\mathrm{T}_{\beta}\right\rangle^{\beta}-\left.\left\langle\mathrm{T}_{\alpha}\right\rangle^{\alpha}\right|^{2}\right) \mathrm{dVdt} \\
& -\oint_{\text {period }} \int_{\mathrm{v}}\left(\varepsilon_{\alpha} \frac{\left\langle\overrightarrow{\mathrm{u}}_{\alpha}\right\rangle^{\alpha}}{\left\langle\mathrm{T}_{\alpha}\right\rangle^{\alpha}} \nabla\left\langle\mathrm{p}_{\alpha}\right\rangle^{\alpha}\right) \mathrm{dVdt} \geq 0
\end{aligned}
$$

\section{Availability Energy Loss:}

It has been noted above that internal entropy generation due to system irreversibilities destroys part of the available work of a system. The destruction of available energy impacts engine thermodynamic performance adversely. Thus, in order to improve the thermodynamic performance of an engineering system it is necessary to study the various mechanisms responsible for entropy generation in order to minimize it in engineering equipment.

All internal entropy generations can be characterized in terms of availability energy loss defined as ${ }^{3,7}$ :

$$
\text { Availabilty energy loss }=\mathrm{T}_{\mathrm{o}} \dot{\mathrm{S}}_{\mathrm{gen}}
$$

The reference temperature $T_{0}$ is the lowest naturally occurring temperature in the system. Evaluation of the external entropy generation equations (22 and 28) and internal entropy generation equations (32 and 36) enable the corresponding availability energy loss calculations using Equation (37). Increase in entropy gives a measure of the extent to which the energy of a system is lost or unavailable for work during a certain process. The direct proportionality between the available energy loss and entropy generation provides a useful gauge to the significance of the various irreversibilities within engineering systems.

The availability-loss concept allows us to think about entropy generation in terms of the more concrete notion of lost mechanical work. A loss in availability equates to a decrease of PV power in an engine. For example, in turbo-machines that generate shaft power (turbines) or absorb power (pumps, compressors), the rate of power lost owing to irreversibilities is proportional to a loss in availability and thus to an increase in the rate of entropy generation.

\section{Second Law Analysis Summary Results:}

The entropy generation results can be summarized as follows:

\section{External Entropy Generation:}

Non-porous domain:

$$
\dot{\mathrm{S}}_{\substack{\text { gen, sys, cyl(ext) } \\ \text { (non-porous) }}}=\oint_{\text {eriod }} \int_{d s}\left(\frac{\overrightarrow{\mathrm{q}}_{\alpha}}{\mathrm{T}_{\alpha}} \cdot \hat{\mathrm{n}}_{\alpha}+\frac{\overrightarrow{\mathrm{q}}_{\beta}}{\mathrm{T}_{\beta}} \cdot \hat{\mathrm{n}}_{\beta}\right) \mathrm{dAdt}+\oint_{\text {period }} \int_{\mathrm{cs}} \rho_{\alpha} \overrightarrow{\mathrm{u}}_{\alpha} \mathrm{s}_{\alpha} \cdot \hat{\mathrm{n}}_{\alpha} \mathrm{dAdt} \geq 0
$$

Porous domain:

$\left\langle\dot{\mathrm{S}}_{\substack{\text { gen,sys, cyl(ext) } \\ \text { porous }}}\right\rangle=\oint_{\text {eriod }} \int_{s}\left(\varepsilon_{\alpha} \mathrm{N}_{\mathrm{k}} \frac{\left\langle\overrightarrow{\mathrm{q}}_{\alpha}\right\rangle^{\alpha}}{\left\langle\mathrm{T}_{\alpha}\right\rangle^{\alpha}} \cdot \hat{\mathrm{n}}_{\alpha}+\frac{\left\langle\overrightarrow{\mathrm{q}}_{\beta}\right\rangle^{\beta}}{\left\langle\mathrm{T}_{\beta}\right\rangle^{\beta}} \cdot \hat{\mathrm{n}}_{\beta}\right) \mathrm{dAdt}+\oint_{\text {period }} \int_{\alpha s} \varepsilon_{\alpha}\left(\left\langle\rho_{\alpha}\right\rangle^{\alpha}\left\langle\mathrm{s}_{\alpha}\right\rangle^{\alpha}\left\langle\overrightarrow{\mathrm{u}}_{\alpha}\right\rangle^{\alpha}\right) \cdot \hat{\mathrm{n}}_{\alpha} \mathrm{dAdt} \geq 0$ 
Internal Entropy Generation:

Non-porous domain:

$$
\dot{\mathrm{S}}_{\text {gen, cyl (int) }}=-\oint_{\text {period }} \int_{\mathrm{cv}}\left(\frac{\overrightarrow{\mathrm{q}}_{\alpha} \cdot \vec{\nabla} \mathrm{T}_{\alpha}}{\mathrm{T}_{\alpha}{ }^{2}}+\frac{\overrightarrow{\mathrm{q}}_{\beta} \cdot \vec{\nabla} \mathrm{T}_{\beta}}{\mathrm{T}_{\beta}{ }^{2}}\right) \mathrm{dV} \mathrm{dt}+\oint_{\text {period }} \int_{\mathrm{cv}} \frac{\Phi}{\alpha} \mathrm{T}_{\alpha} \mathrm{dV} \mathrm{dt} \geq 0
$$

where

$$
\begin{aligned}
\frac{\overrightarrow{\mathrm{q}} \cdot \vec{\nabla} \mathrm{T}}{\mathrm{T}^{2}} & =\frac{\mathrm{k}}{\mathrm{T}^{2}}\left[\left(\frac{\partial \mathrm{T}}{\partial \mathrm{x}}\right)^{2}+\left(\frac{\partial \mathrm{T}}{\partial \mathrm{y}}\right)^{2}+\left(\frac{\partial \mathrm{T}}{\partial \mathrm{z}}\right)^{2}\right] \\
\frac{\Phi}{\mathrm{T}} & =\frac{1}{\mathrm{~T}}\left\{(-2 / 3) \mu(\vec{\nabla} \cdot \overrightarrow{\mathrm{u}})^{2}+2 \mu\left[\left(\frac{\partial \mathrm{u}}{\partial \mathrm{x}}\right)^{2}+\left(\frac{\partial \mathrm{v}}{\partial \mathrm{y}}\right)^{2}+\left(\frac{\partial \mathrm{w}}{\partial \mathrm{z}}\right)^{2}\right]+\mu\left[\left(\frac{\partial \mathrm{v}}{\partial \mathrm{x}}+\frac{\partial \mathrm{u}}{\partial \mathrm{y}}\right)^{2}+\left(\frac{\partial \mathrm{w}}{\partial \mathrm{y}}+\frac{\partial \mathrm{v}}{\partial \mathrm{z}}\right)^{2}+\left(\frac{\partial \mathrm{u}}{\partial \mathrm{z}}+\frac{\partial \mathrm{w}}{\partial \mathrm{x}}\right)^{2}\right]\right\}
\end{aligned}
$$

Porous domain:

$$
\begin{aligned}
& \left\langle\dot{\mathrm{S}}_{\text {gen,cyl(int.) }}\right\rangle=\oint_{\text {period }} \int_{\mathrm{vv}}\left\{\varepsilon_{\alpha} \mathrm{N}_{\mathrm{k}} \mathrm{k}_{\alpha}\left(\frac{\nabla\left\langle\mathrm{T}_{\alpha}\right\rangle^{\alpha}}{\left\langle\mathrm{T}_{\alpha}\right\rangle^{\alpha}}\right)^{2}+\varepsilon_{\beta} \mathrm{k}_{\beta}\left(\frac{\nabla\left\langle\mathrm{T}_{\beta}\right\rangle^{\beta}}{\left\langle\mathrm{T}_{\beta}\right\rangle^{\beta}}\right)^{2}\right\} \mathrm{dVdt}+\oint_{\text {period }} \int_{\mathrm{vv}}\left(\frac{\varepsilon_{\alpha} \alpha_{v} \mathrm{H}}{\left\langle\mathrm{T}_{\alpha}\right\rangle^{\alpha}\left\langle\mathrm{T}_{\beta}\right\rangle^{\beta}}\left|\left\langle\mathrm{T}_{\beta}\right\rangle^{\beta}-\left\langle\mathrm{T}_{\alpha}\right\rangle^{\alpha}\right|^{2}\right) \mathrm{dVdt} \\
& -\oint_{\text {eriod }} \int_{\alpha v}\left(\varepsilon_{\alpha} \frac{\left\langle\overrightarrow{\mathrm{u}}_{\alpha}\right\rangle^{\alpha}}{\left\langle\mathrm{T}_{\alpha}\right\rangle^{\alpha}} \nabla\left\langle\mathrm{p}_{\alpha}\right\rangle^{\alpha}\right) \mathrm{dVdt} \geq 0
\end{aligned}
$$

Availability Energy Loss:

$$
\text { Availabilty energy loss }=\mathrm{T}_{\mathrm{L}} \dot{\mathrm{S}}_{\mathrm{gen}}
$$

Loss analysis using entropy-generation rates due to heat transfer, viscous friction and non-equilibrium processes is a relatively new technique for assessing component performance. It offers a deep insight into the flow phenomena, allows a more exact calculation of losses than is possible with traditional means involving the application of loss correlations and provides an effective tool for improving performance. Entropy generation maps of cumulative amounts of all losses computed locally in the flow domain can be produced and designers can use them to detect critical areas (locations in which entropy generation is higher than its integral average value over the entire flow field). The design emphasis would then be aimed at reducing the local values for entropy production in these critical areas by modifying design variables so as to improve the overall performance.

Our understanding of loss mechanisms is however far from complete. Although numerical predictions are valuable in predicting the heat transfer and flow structure, there are difficulties in predicting the loss accurately. This is due to errors in predicting the boundary layers, and laminar/turbulent transition, as well as due to false entropy generation due to numerical dissipation. This work provides a point of reference for incorporation of loss postprocessors into Stirling engine numerical codes. The incorporation of a loss post-processor in Stirling engine numerical codes, it is believed, will facilitate the optimization of Stirling engine performance. 


\section{Numerical Simulation Results}

In a prior study ${ }^{12}$, extensive numerical simulations of the fluid flow and heat transfer phenomena under conditions of oscillating pressure and oscillating fluid flow inside the original MIT 2-space solution domain were performed using 1-D Sage and 2-D CFD-ACE+ numerical codes. In this study, 1-D Sage and 2-D Fluent numerical codes are used to obtain numerical simulation results in the Run F configuration (see Table 1) of the 3-space solution domain. As discussed in Section III, the optimum length of the regenerator is shown in Figure 6 to be $20 \mathrm{~cm}$. (with a corresponding $24.5 \mathrm{~cm}$ heat exchanger length). For 2-D numerical simulations in both prior and current studies, the grid size, numbers of cycles of piston motion and time step were optimized prior to performing the simulations, as detailed in the prior study ${ }^{12}$. The thermal non-equilibrium porous-media models discussed in Section IV can be employed for the numerical simulations of the fluid flow and heat transfer effects in the regenerator. However, some difficulties were encountered incorporating the derived models in Section IV in the Fluent code. Following a series of consultations with Fluent Inc., a viable alternative for performing thermal non-equilibrium analysis was suggested. This entails using the TGrid feature in Fluent to generate a volume mesh of the solid phase and separating it from existing boundary mesh of the regenerator domain resulting in two volume meshes - a solid volume mesh and a fluid volume mesh. The film heat transfer term in Eq. (17) is then employed as the source term to link the two phases. This suggested approach was adopted in this study.

Code (1D vs. 2D) and model (2-space vs. 3-space) simulation results of pressure-volume diagrams and crank angle dependent temperature, pressure and wall heat transfer profiles are compared. To ensure a common basis for comparison, the reference point for the piston motion is taken at $\operatorname{BDC}\left(\theta=0^{\circ}\right)$ moving toward TDC. Also, results of heat exchanger surface heat fluxes, temperature differences between the gas temperature at the radial center of the heat exchanger and the heat exchanger wall, domain temperature contours and velocity vectors generated by the $2 \mathrm{D}$ codes are compared with similar results obtained from the literature ${ }^{20,27,36}$. For each comparison, important differences and similarities and the effect(s) of the regenerator are noted and discussed.

\section{Code (1D vs. 2D) and Model (2-space vs. 3-space) Comparisons}

\subsection{Pressure-Volume Diagrams}

2-space model

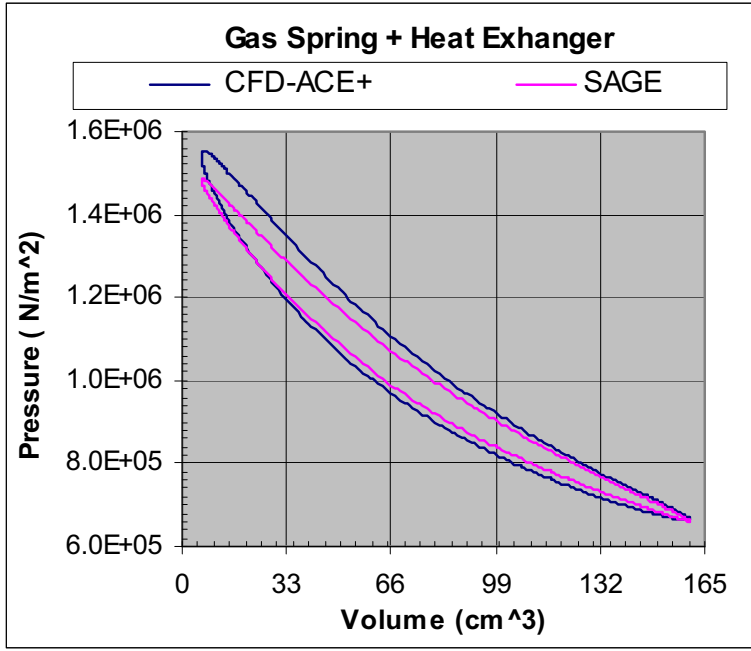

3-space model

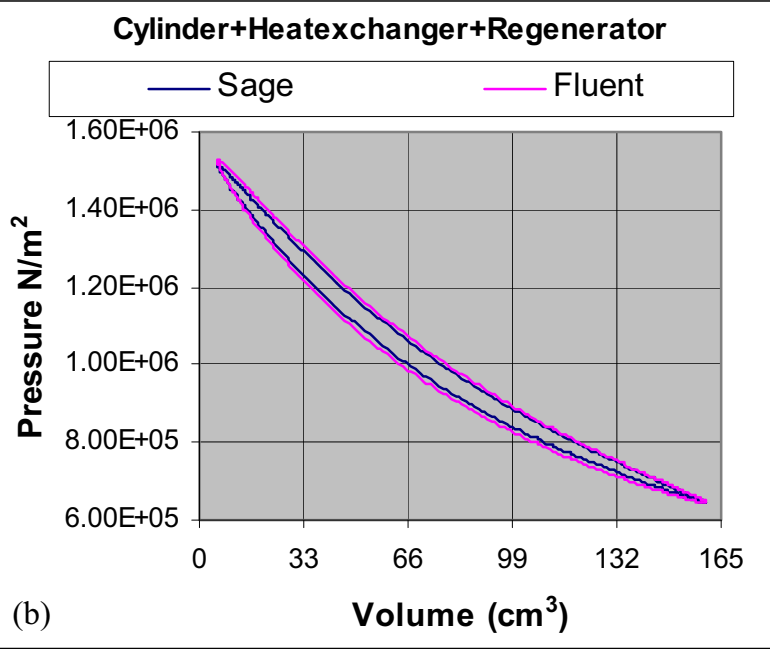

Figure 8. Pressure-Volume Diagrams over a Cycle in the Cylinder Space (Operating Conds.: 201.7 RPM, 1.008 MPa., $\mathrm{T}_{\mathrm{w}}=294 \mathrm{~K}, 480$ tspc, Grid size: 147 x 46).

Pressure-volume diagrams over a cycle in the variable volume cylinder space of the 2-space and 3-space domains are illustrated in Figs. 8(a,b). The indicated operating pressure is the arithmetic mean (of the compression and expansion space pressures) cycle pressure. Wall and piston-face temperature was fixed at $294 \mathrm{~K}$. The pressurevolume diagrams appear qualitatively similar. Note that the maximum cylinder volume $\left(160.4 \mathrm{~cm}^{3}\right)$, the minimum cylinder volume $\left(5.9 \mathrm{~cm}^{3}\right)$ and the swept volume $\left(154.5 \mathrm{~cm}^{3}\right)$ are the same in both models and that the maximum pressure in each cylinder space is on the average about 2.3 times the minimum pressure. Calculations of maximum 
pressures at minimum compression volume compare better $(\sim 0.6 \%$ error $)$ in the 3 -space model than in the 2 -space model $(\sim 4.3 \%$ error $)$. On the other hand, the minimum pressures at maximum expansion volume compare slightly better for the 2 -space model ( $\sim 0.3 \%$ error) than for the 3 -space model $(\sim 0.4 \%$ error $)$. On the average, Sage and Fluent pressure results appear to compare better than Sage and CFD-ACE+ pressure results.

The areas enclosed by the $\mathrm{P}-\mathrm{V}$ diagrams are each equivalent to a net piston work input because the work done by the system on the piston during the expansion process is less than the work done on the system by the piston during the compression part of the cycle. The discrepancy between 1D and 2D calculations of the piston net work input in each model was significant ( $\sim 4 \%$ error). The 1D Sage code under predicts the piston net work input. The inclusion of the regenerator resulted in a reduction in the minimum and maximum pressure and work-input values in the cylinder with the exception of the increase in Sage maximum pressure.

\subsection{Crank Angle dependent Temperature and Pressure Profiles}

2-D instantaneous temperature and pressure data are obtained at specified points (stationary and/or moving) in the domain of interest over a cycle of piston motion defined using crank angle, $\theta$, as:

$$
\text { Crank Angle, } \theta=[(\omega \mathrm{t}+3 \pi / 2) \cdot 180 / \pi+270] / 360
$$

The parameter $\omega$ is angular frequency and $t$ is time. The use of crank angle enables comparison at the same piston position.

Sage employs the equation

$$
\Phi_{i}=\bar{\Phi}+\sum_{n=1}^{3} A_{n} \cos \left(n \omega t_{i}+\varphi\right), \quad i=1,2, \ldots, \quad \# \text { tspc }
$$

to calculate the instantaneous values, $\Phi_{i}$, of the data of interest, $\Phi$, (temperature or pressure) over the number of time steps per cycle (\#tspc). Each of the instantaneous values $\Phi_{i}$ is equal to the sum of the mean value, $\bar{\Phi}$, and three Fourier cosine harmonics of the piston motion at each time step. The parameter $A$ is the amplitude of $\Phi$ and $\varphi$ is the phase angle. $\bar{\Phi}$; A and $\varphi$, which are spatially averaged values over the domain are obtained from Sage output results.

In the cylinder space, temperature and pressure data are obtained at specified stationary points (x,y) and moving points (x-node, y-node) and in the heat exchanger and regenerator spaces, temperature and pressure data are obtained at specified stationary points only. Note that $\mathrm{x}, \mathrm{y}$-node numbers are used as coordinates to tag the moving point location. The y-coordinate $(0.0132673 \mathrm{~m})$ or y-node $(9)$, for example, is fixed in each domain.

The cylinder space temperature profiles for three stationary points and three moving grid points are illustrated in Figs. 9(a-d) below. The x-coordinates (or x-nodes) of the points are as shown in the Figures.

The cylinder space temperature profiles are observed to be dependent on point location and on whether the point is stationary or moving. Profile irregularity is more pronounced for stationary points than for moving points and more especially for stationary points close to the midpoint of the cylinder clearance volume $(\mathrm{x}=0.001374 \mathrm{~m})$ and the piston top center position $(x=0.002747 \mathrm{~m})$. The sudden dip in temperature at $\theta=180^{\circ}$ for the stationary point at $\mathrm{x}=0.002747 \mathrm{~m}$. may possibly be due to flow disturbances close to the piston top center position. All CFD$\mathrm{ACE}+$ /Fluent generated peak temperatures for stationary and moving points are below Sage's peak temperature. Sage over predicts the highest temperatures recorded by the 2-D codes in the 2-space and 3-space models by $\sim 16 \mathrm{~K}$ and $\sim 11 \mathrm{~K}$ respectively. In general, temperature values are observed to increase with crank angle during the compression phase and decrease during the expansion phase of the cycle as expected. Close to the cylinder head or entrance to the heat exchanger $(x=0.000153 \mathrm{~m}, \mathrm{x}$-node 2$)$, the fluid in the cylinder space experiences an appreciable drop in the peak temperature since almost all the fluid is pushed into the heat exchanger at this point. Addition of the regenerator elevates the cylinder space temperatures at stationary points especially at points close to the midpoint of the cylinder clearance volume $(\mathrm{x}=0.001374 \mathrm{~m})$ and piston top center position $(\mathrm{x}=0.002747 \mathrm{~m})$. 


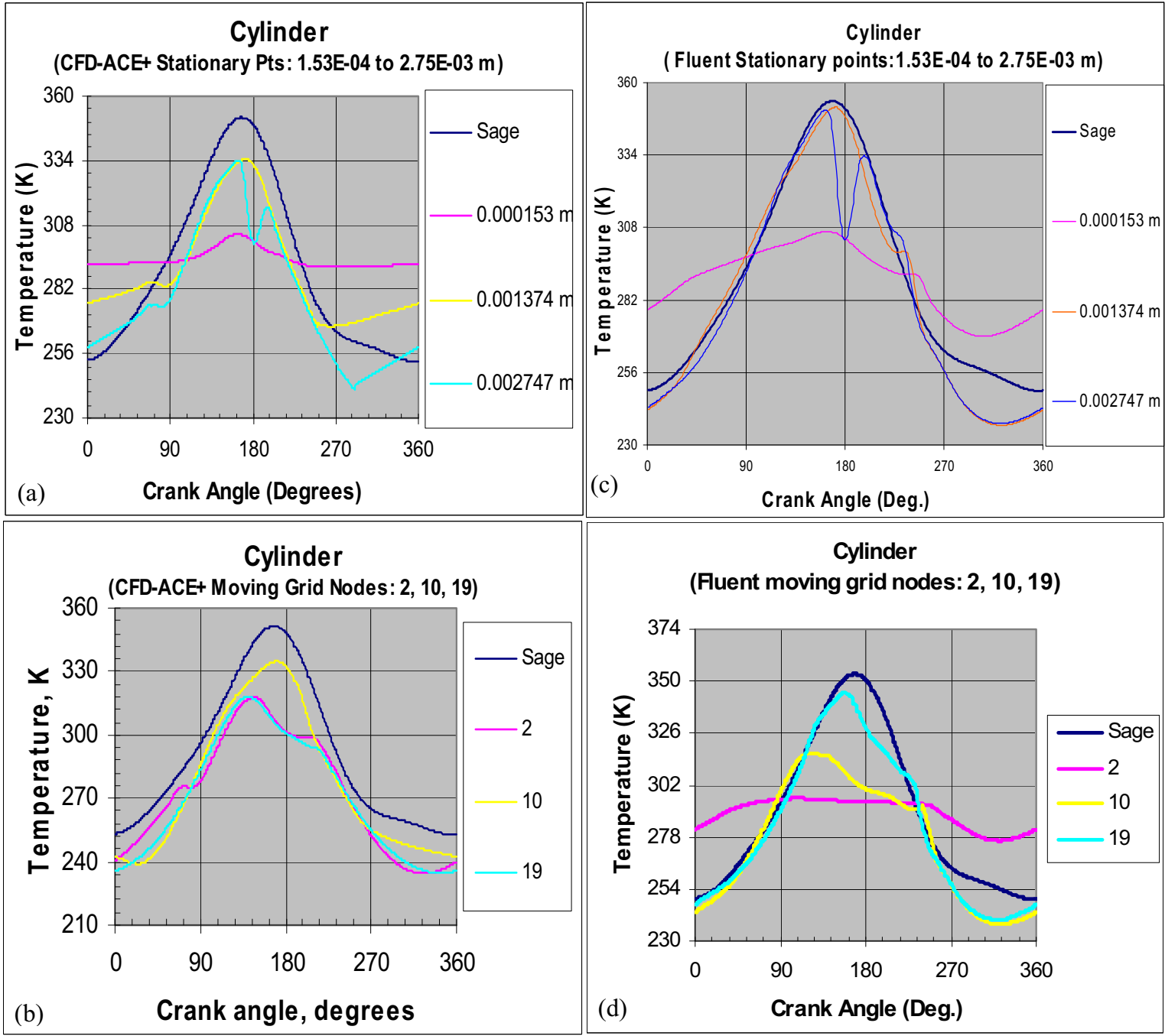

Figures 9(a-d). Temperature Profiles at Stationary and Moving Points in Cylinder Space (Operating Conds.: 201.7 RPM, 1.008 MPa, T wall $_{1} 294 \mathrm{~K}$, \#tspc = 480, Grid size = $147 \mathrm{x} 46$ ).

Temperature profiles for the stationary points in the heat exchanger space are illustrated in Figs. 9(e,f). Five and three stationary points are specified for the 2-space and 3-space models respectively. Only three stationary points are specified in the heat exchanger space of the 3-space model because of its reduced length due to the incorporation of the regenerator. Profile irregularity is pronounced during the compression part of the cycle for points at or close to the heat exchanger entrance $(0.0$ and $-0.11 \mathrm{~m})$. As expected, maximum temperatures are recorded near the end of the compression process and minimum temperatures recorded close to the end of the expansion process. Unlike in the cylinder space, peak temperatures in the heat exchanger recorded by the 2-D codes are greater than Sage's peak temperature $\left(\sim 304 \mathrm{~K} @ \theta \sim 148^{\circ}\right)$ and in this case, all minimum temperatures recorded by the 2-D codes are lower than or about equal to Sage's minimum temperature $\left(\sim 286 \mathrm{~K} @ \theta \sim 274^{\circ}\right)$. For the 2-space model, the highest temperature $\left(\sim 344.1 \mathrm{~K} @ \theta \sim 146.3^{\circ}\right)$ and lowest temperature $\left(\sim 260.8 \mathrm{~K} @ \theta \sim 287.3^{\circ}\right)$ are recorded near the end of the heat exchanger $(\mathrm{x}=-0.33 \mathrm{~m})$ disregarding the anomaly near the heat exchanger entrance. For the 3-space model, the highest temperature $\left(\sim 333 \mathrm{~K} @ \theta \sim 135^{\circ}\right)$ and lowest temperature $\left(\sim 262.8 \mathrm{~K} @ \theta \sim 270^{\circ}\right)$ are recorded near the heat exchanger entrance $(\mathrm{x}=0 \mathrm{~m}$.). 
2-space model

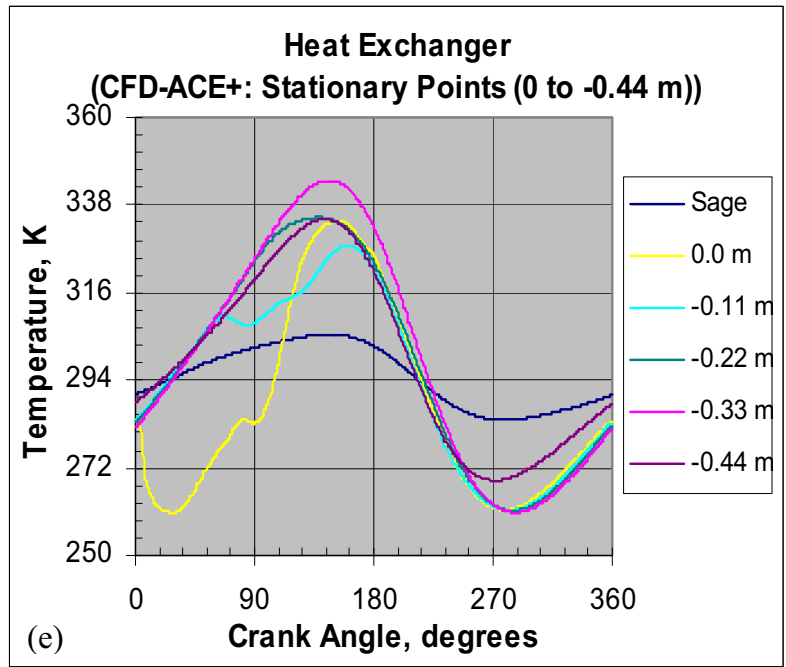

3-space model

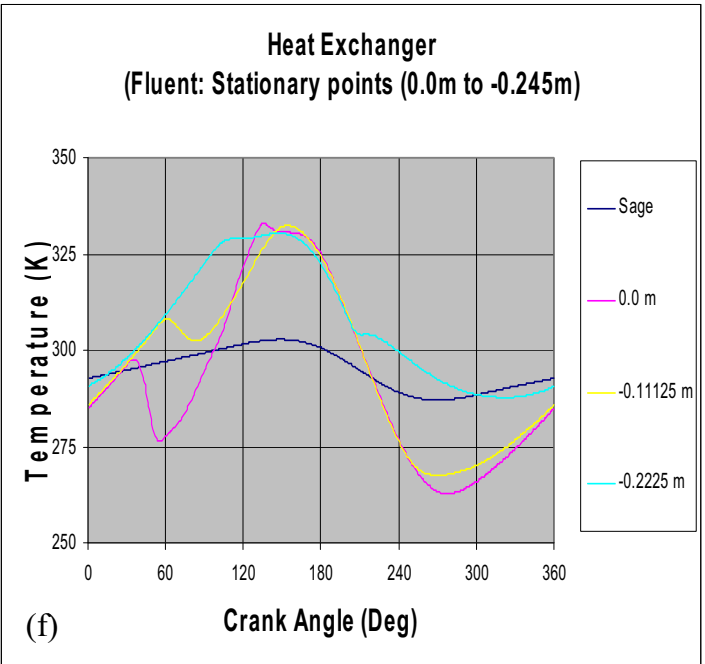

(f)

Figure 9(e-f). Temperature Profiles at Stationary Points.

(Operating Conds.: $201.7 \mathrm{RPM}, 1.008 \mathrm{MPa}, \mathrm{T}_{\text {wall }}=294 \mathrm{~K}$, \#tspc $=480$, Grid size $=147 \mathrm{x} 46$ ).

The 2-D codes peak temperatures are greater than Sage's by $\sim 40 \mathrm{~K}$ (2-space) and $\sim 29 \mathrm{~K}$ (3-space). The Sage code on the other hand is greater than the 2-D codes' minimum temperature by $\sim 25 \mathrm{~K}$ (2-space) and $\sim 23 \mathrm{~K}$ (3-space). The presence of the regenerator does appear to decrease the maximum temperature recorded in the heat exchanger of the 2-space model by $\sim 11 \mathrm{~K}$, and increase the minimum temperature by $\sim 2 \mathrm{~K}$; it also shifts the maximum and minimum temperature values from near the end of the heat exchanger to near the heat exchanger entrance during a cycle.

The cylinder space and heat exchanger space pressure profiles are illustrated in Figs. 9(g-j). The pressure profiles in the cylinder and heat exchanger spaces are relatively insensitive to whether stationary or moving spatial coordinates are used; they are fairly symmetric over the crank angle range $0^{\circ} \leq \theta \leq 360^{\circ}$ with peak pressure values predictably occurring very close to the end of the compression phase of the cycle. In both cylinder and heat exchanger domains, Fluent and Sage pressure profiles are in excellent agreement over all crank angles for the 3space model with maximum pressure $1.55 \mathrm{E}+06 \mathrm{~N} / \mathrm{m}^{2}$ occurring at $\theta \approx 175^{\circ}$. For the 2 -space model, CFD-ACE + and Sage pressure profiles are not in agreement over all crank angles in the cylinder and heat exchanger domains. In the cylinder domain, the lack of correspondence is over the crank angle range $90^{\circ} \leq \theta \leq 210^{\circ}$ and in the heat exchanger domain, there is a significant difference between CFD-ACE + and Sage pressure predictions. Correspondence is observed only at two crank angles $\left(\theta \approx 81^{\circ}\right.$ and $\left.\theta \approx 261^{\circ}\right)$. At maximum compression $\left(\theta \approx 175^{\circ}\right)$, CFD-ACE+'s peak pressure prediction exceeds Sage's by about $60.0 \mathrm{kPa}$ with about $1.8^{\circ}$ crank angle lead in the cylinder and by about $420.0 \mathrm{kPa}$ with about $3.3^{\circ}$ crank angle lead in the heat exchanger. It is also observed that whereas peak pressure values predicted by Fluent in the 3-space domains (cylinder and heat exchanger) are less than those predicted by CFD-ACE+ in the corresponding 2-space domains, peak pressure values predicted by Sage in the cylinder and heat exchanger domains are higher for the 3-space model. Note also that in going from the cylinder space to the heat exchanger space, Sage predicts a large pressure drop $\left(\sim 0.36 \mathrm{E}+06 \mathrm{~N} / \mathrm{m}^{2}\right)$. CFD-ACE + /Fluent predicts no pressure drop. The argument can be made that the addition of the regenerator helps to improve the pressure profile correspondence with the Sage result in both the heat exchanger and cylinder spaces and to reduce the 2-D peak pressure values in both the cylinder and heat exchanger domains. 
2-space model
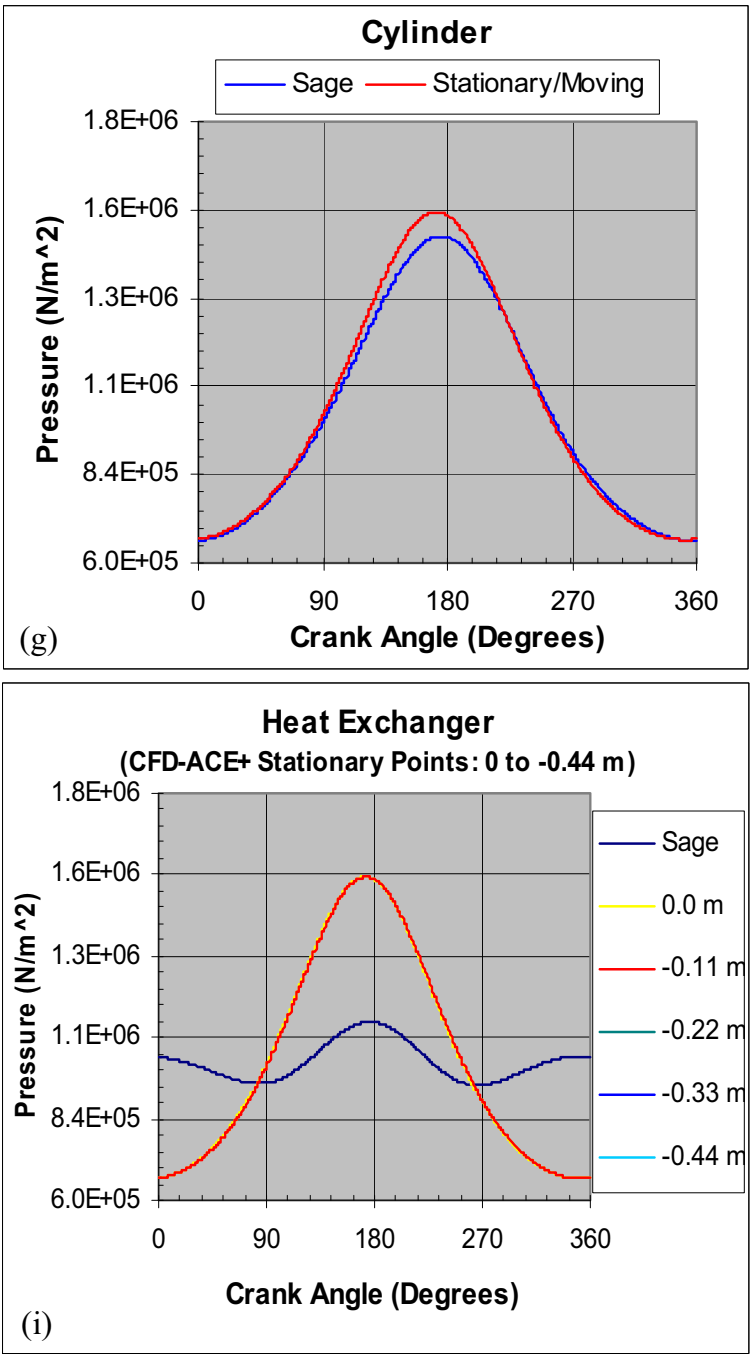

3-space model
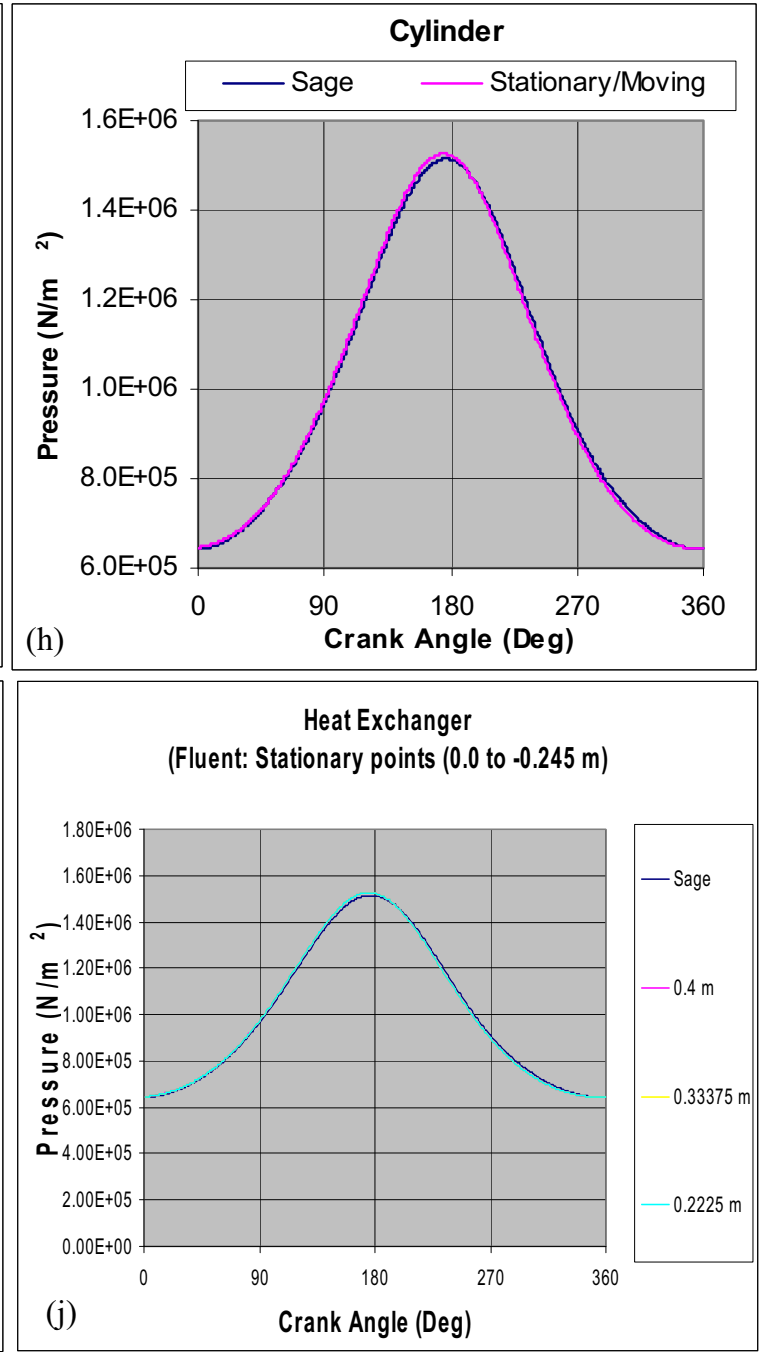

Figure 9(g-j). Pressure Profiles at Stationary and/or Moving Points.

(Operating Conds.: 201.7 RPM, 1.008 MPa, $\mathrm{T}_{\text {wall }}=294 \mathrm{~K}$, \#tspc = 480, Grid size = $147 \mathrm{x} 46$ ).

The pressure profiles in the regenerator are illustrated in Figure 10. Pressure profiles at two stationary points are recorded by the Fluent code. Fluent pressure profiles are insensitive to point location and are in excellent agreement with the pressure profile recorded by Sage over the crank angle range $0^{\circ} \leq \theta \leq 360^{\circ}$. The maximum pressure of about $1.5 \mathrm{E}+06 \mathrm{~N} / \mathrm{m}^{2}$ occurs at $\theta \approx 175^{\circ}$.

Regenerator temperature profiles over the crank angle range $0^{\circ} \leq \theta \leq 360^{\circ}$ predicted using Fluent and Sage are illustrated in Figure 11. The fluid and solid temperature profiles predicted by Sage are in phase, each with very little temperature variation - only $\sim 0.8 \mathrm{~K}$ around $\sim 339.8 \mathrm{~K}$. On the other hand, the fluid and solid temperature profiles predicted by Fluent are out of phase. The fluid temperature profile fluctuates about $40 \mathrm{~K}$ around $\sim 320 \mathrm{~K}$ and the solid temperature profile fluctuates about $37 \mathrm{~K}$ around $318 \mathrm{~K}$. There is clearly poor agreement between Sage and Fluent predictions. The arbitrariness of some of the prediction parameters (e.g., density and thermal conductivity of the solid matrix) introduced into the Fluent code in order to achieve the thermal non-equilibrium condition may be responsible for the disagreement between Fluent and Sage. As expected, the regenerator solid is seen to store heat 
during the compression phase of the cycle (during which the fluid temperature is higher than the temperature of the solid) and to release heat to the fluid during the expansion phase of the cycle (during which the fluid temperature is lower than the temperature of the solid).

\section{Regenerator in 3-space domain}

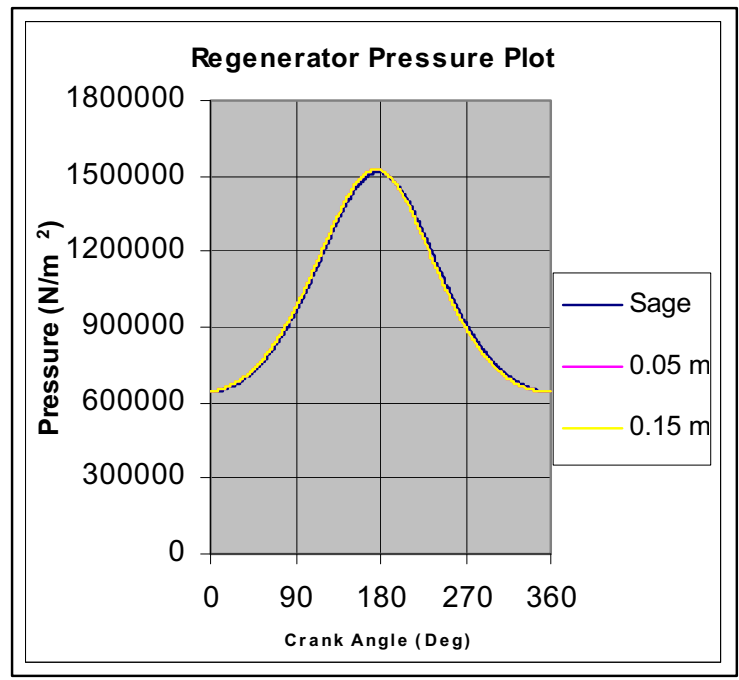

Figure 10. Temperature and Pressure Profiles at Stationary Points.

(Operating Conds.: 201.7 RPM, 1.008 MPa, $\mathrm{T}_{\text {wall }}=294 \mathrm{~K}$, \#tspc $=480$, Grid size $=147 \mathrm{x} 46$ ).

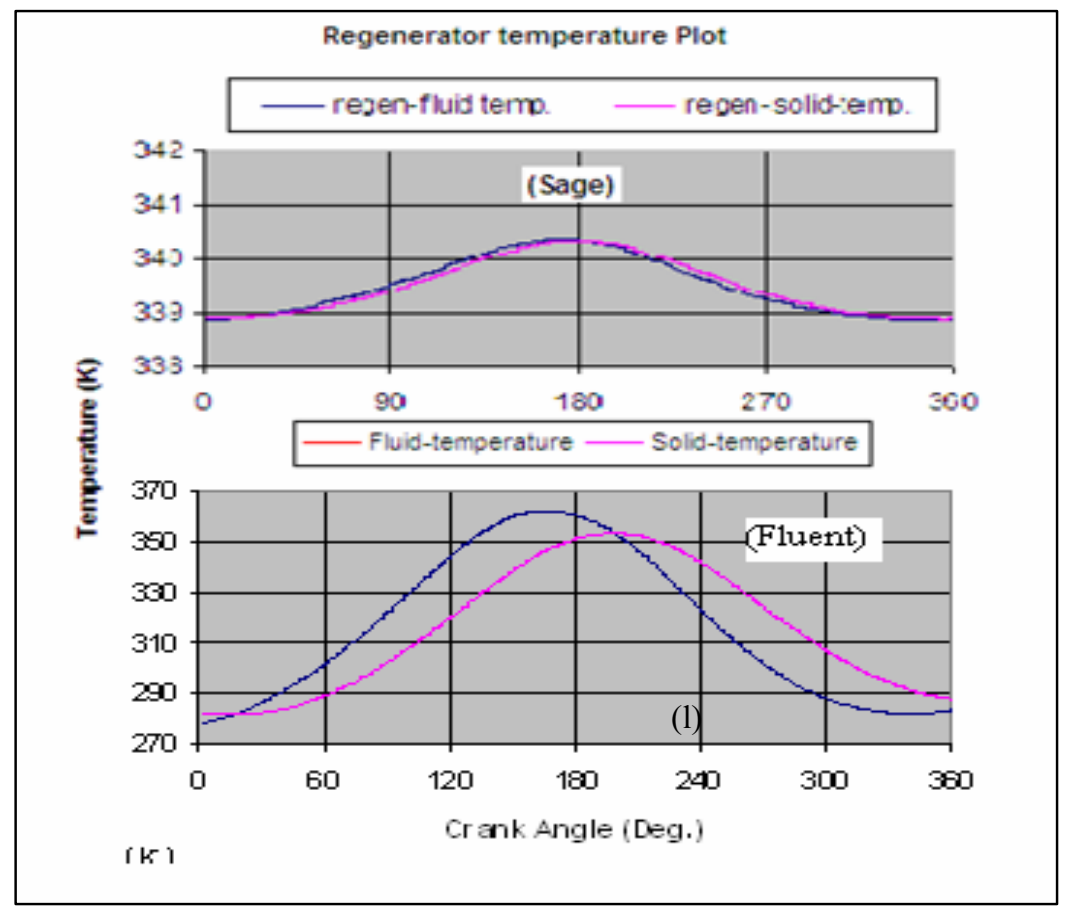

Figure 11. Temperature and Pressure Profiles at Stationary Points.

(Operating Conds.: 201.7 RPM, 1.008 MPa, $\mathrm{T}_{\text {wall }}=294 \mathrm{~K}$, \#tspc = 480, Grid size $=147 \mathrm{x} 46$ ). 


\subsection{Wall Heat Transfer Rate}

From the energy conservation principle expressed in differential form per unit time for a closed system undergoing a cyclic process:

$$
\oint \delta \dot{\mathrm{Q}}=\oint \delta \dot{\mathrm{W}}
$$

The expansion and compression work of the piston $\delta \dot{\mathrm{W}}$ is a moving boundary work or pdV work where $\mathrm{p}$ is pressure and $\mathrm{dV}$ is differential volume. For non-quasi-equilibrium processes such as we have, the pressure at the inner face of the piston $p_{i}$ is used for $p$. Thus the equation relevant to our study is

$$
\frac{1}{\mathrm{~T}} \oint \delta \mathrm{Q}=\frac{1}{\mathrm{~T}} \oint \mathrm{p}_{\mathrm{i}} \mathrm{dV}
$$

The parameter $\mathrm{T}$ is the period of the cycle. In CFD-ACE + /Fluent, $\oint \delta \mathrm{Q} / \mathrm{T}$ is calculated by first summing the heat transfer $(\mathrm{Q})$ input data over the total number of time steps in a cycle and then dividing the sum by the period $\mathrm{T}$ and $\int \mathrm{p}_{\mathrm{i}} \mathrm{dV} / \mathrm{T}$ is computed using Simpson's integration rule over the total number of time steps in a cycle viz:

$$
\frac{1}{T} \oint p_{i} d V=\frac{1}{T}\left[\sum_{n=1}^{\# t s p c}\left(\frac{p_{i}{ }^{n}+p_{i}{ }^{n-1}}{2}\right)\left(V^{n}-V^{n-1}\right)\right]
$$

The superscripts $\mathrm{n}$ and $\mathrm{n}-1$ imply values at the current and previous time steps respectively. The volume change $\left(\mathrm{V}^{\mathrm{n}}-\mathrm{V}^{\mathrm{n}-1}\right)$ is calculated by multiplying the piston displacement during the given time step by the piston area. Sage output results provide mean values of the heat transfer rate and pressure which are equivalent to the integral parameters $\oint \delta \mathrm{Q} / \mathrm{T}$ and $\oint \mathrm{p}_{\mathrm{i}} \mathrm{dV} / \mathrm{T}$ respectively.

Recktenwald ${ }^{27}$ modified Eq. (49) by introducing the work term weighting factor $\beta$ to reduce the error introduced by the discretization of the pressure-volume work term viz.:

$$
\oint \mathrm{p}_{\mathrm{i}} \mathrm{dV}=\sum_{\mathrm{n}=1}^{\# \text { tspc }}\left[\beta \mathrm{p}_{\mathrm{i}}{ }^{\mathrm{n}}+(1-\beta) \mathrm{p}_{\mathrm{i}}{ }^{\mathrm{n}-1}\right]\left(V^{n}-V^{n-1}\right)
$$

The discretization is controlled by adjusting $\beta$ to obtain cycle energy balance. As with the 2 -space model, a $\beta$ value of 1.49 was the optimum obtained for the 3 -space model. Table 6 shows work and heat transfer calculation results

\begin{tabular}{|c|c|c|c|c|c|c|c|c|}
\hline \multirow[b]{2}{*}{ Code } & \multicolumn{3}{|c|}{ 2-space model } & \multirow{2}{*}{ Code } & \multicolumn{4}{|c|}{ 3-space model } \\
\hline & $\begin{array}{l}\text { Net Input } \\
\text { Work, W }\end{array}$ & \multicolumn{2}{|c|}{ Heat Transfer, W } & & $\begin{array}{l}\text { Net Input } \\
\text { Work, W }\end{array}$ & \multicolumn{3}{|c|}{ Heat Transfer, W } \\
\hline \multirow{2}{*}{ Sage } & \multirow{2}{*}{29.95} & $\begin{array}{c}\dot{\mathrm{Q}}_{\text {out }} \\
\text { (HXer.) }\end{array}$ & $\begin{array}{l}\dot{\mathrm{Q}}_{\mathrm{in}} \\
\text { (Cyl.) }\end{array}$ & \multirow{2}{*}{ Sage } & \multirow{2}{*}{22.37} & $\begin{array}{c}\dot{\mathrm{Q}}_{\text {out }} \\
\text { (Regen.) }\end{array}$ & $\begin{array}{c}\dot{\mathrm{Q}}_{\text {out }} \\
\text { (HXer.) }\end{array}$ & $\begin{array}{l}\dot{\mathrm{Q}}_{\text {in }} \\
\text { (Cyl.) }\end{array}$ \\
\hline & & 42.58 & 12.71 & & & $1.797 \mathrm{E}-07$ & 37.95 & 15.58 \\
\hline $\begin{array}{l}\text { CFD- } \\
\text { ACE+ }\end{array}$ & 53.24 & 80.93 & 27.65 & Fluent & 34.12 & 0.0 & 54.07 & 19.93 \\
\hline
\end{tabular}
for a single cycle taken from mid $6^{\text {th }}$ cycle to mid $7^{\text {th }}$ cycle.

Table 6. Sage and CFD-ACE+/Fluent Work and Heat Transfer Data (with the incorporation of the $\beta$ weighting factor)

Note that work and heat transfer calculations must be multiplied by two to get values for the entire domain since only one half of the domain is simulated. 
The cylinder input heat rate corresponds to a rate of cooling of the cylinder walls. The heat exchanger output heat rate corresponds to the heating rate of the heat exchanger wall. As expected, the results indicate no energy is stored within the regenerator matrix during a cycle of operation. Generally, the heat stored in the regenerator matrix during one-half of the cycle is removed during the other half of the cycle. The net work input, $\dot{\mathrm{W}}_{\text {net, in }}=\dot{\mathrm{Q}}_{\text {out }}-\dot{\mathrm{Q}}_{\text {in }}$ is confirmed by the results in Table 7. The piston action essentially pumps heat from the cylinder to the heat exchanger and regenerator. Thus with respect to the cylinder, the model acts like a "cooler" whereas with respect to the heat exchanger, the model acts as a "heat pump" even though the wall temperature is kept constant at $294 \mathrm{~K}$.

With the addition of the regenerator, Sage reports $\sim 23 \%$ increase in the cooling action in the cylinder, $\sim 11 \%$ reduction in the heat pumping action in the heat exchanger and $\sim 25 \%$ reduction in the net cycle heat loss (or input work) is noted. On the other hand, Fluent, in relation to the CFD-ACE+ 2-space calculations, reports $\sim 28 \%$ reduction in the cooling action in the cylinder (though the COP increases by $\sim 12 \%$ ), $\sim 33 \%$ reduction in the heat pumping action in the heat exchanger and $\sim 36 \%$ reduction in the net cycle heat loss (or input work) is noted. Thus the addition of the regenerator leads to a reduction in the cylinder cooling action, heat pumping action and the net cycle heat loss (or work input) but an increase in COP.

The heat addition-heat rejection process described above occurs in Stirling engines also. Heat is pumped from the expansion volume of a Stirling engine to the "appendix gap" (clearance volume between the displacer piston and the cylinder) and is lost to the walls of the clearance volume. This represents a net loss of heat to the work producing portion of the Stirling engine and is called an "appendix gap pumping loss".

Figures 12(a-d) show plots of wall heat transfer rate as a function of the crank angle. Cylinder wall, heat exchanger wall and total wall heat transfer rates are illustrated in Figs. 12(a,b) for the "2-space" model. The regenerator wall heat transfer rate is added in Figs. 12(c,d) for the "3-space" models.

2-space model

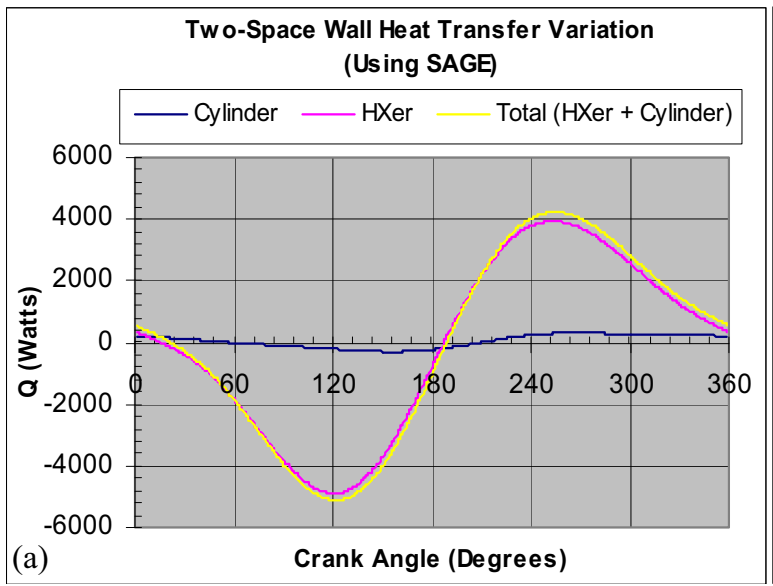

\section{Two-Space Wall Heat Transfer Variation}

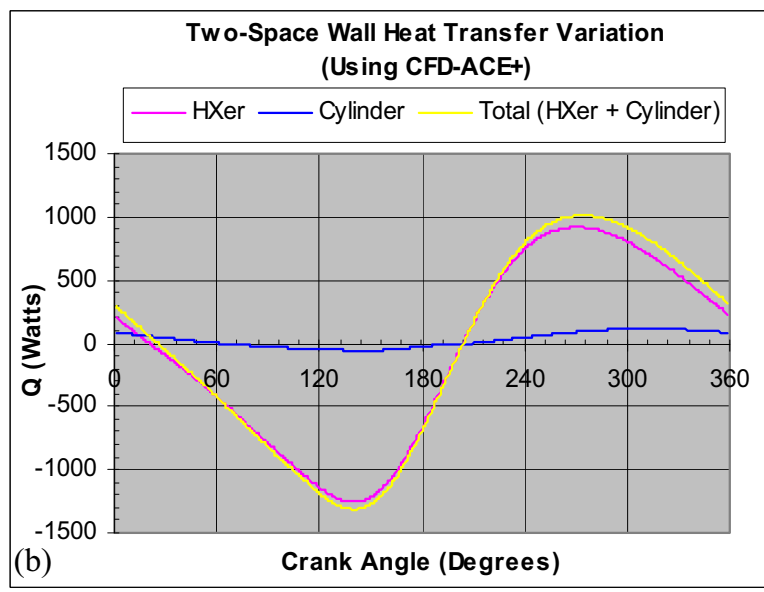

3-space model
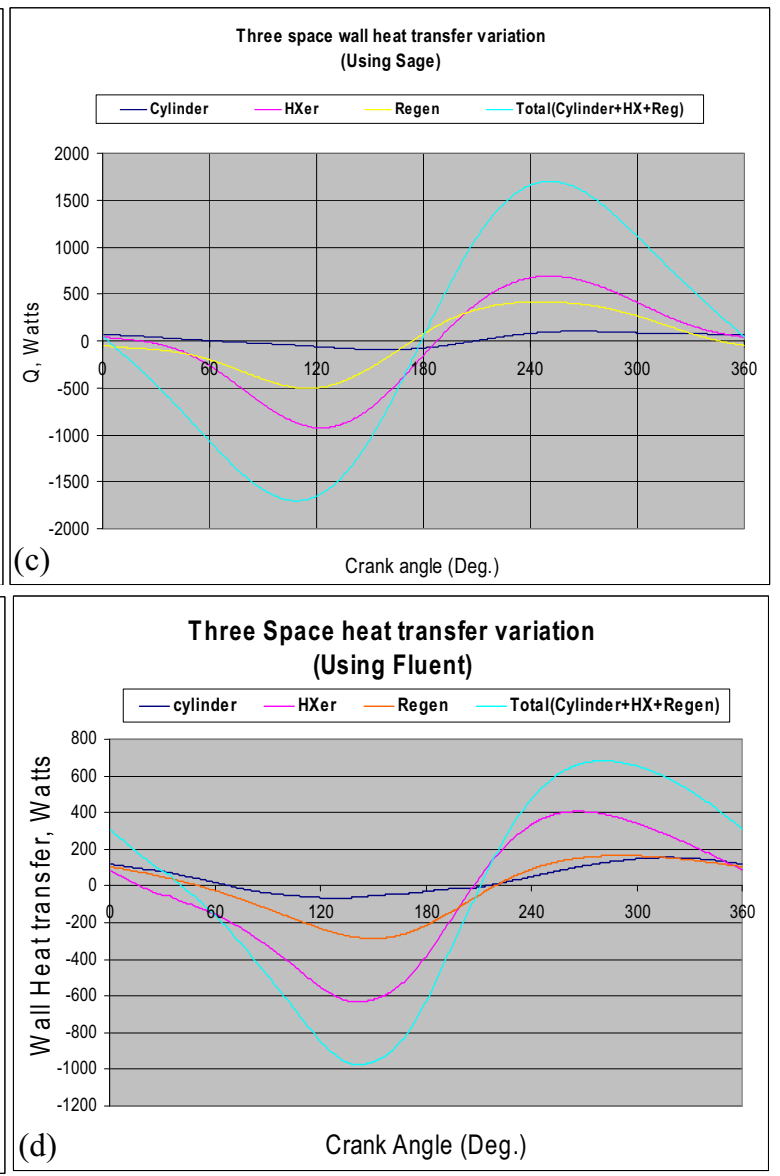

Figure 12(a-d). Wall Heat Transfer Vs. Crank Angle

(Operating Conds.: 201.7 RPM, 1.008 MPa, $\mathrm{T}_{\text {wall }}=294 \mathrm{~K}$, \#tspc $=480$, Grid size $=147 \mathrm{x} 46$ ) 
Plots of the wall heat transfer rates exhibit oscillatory behavior over the range $0^{\circ} \leq \theta \leq 360^{\circ}$ in all the domains. It is observed that CFD-ACE+/Fluent and Sage signals are not in phase. In the 2-space model, the difference in the net cycle heat loss (or work input) between CFD-ACE+ $(53.24 \mathrm{~W})$ and Sage $(29.95 \mathrm{~W})$ predictions is $\sim 43.7 \%$. In the 3-space model, the difference in the net cycle heat loss (or work input) between Fluent (34.12 W) and Sage $(22.37 \mathrm{~W})$ predictions is $\sim 34.4 \%$. In each percent difference calculation, the 2-D code is used as the basis for accuracy. Note that these results are consistent with corresponding entries in Table 6 . The incorporation of the regenerator tended to reduce the error between the 1-D and 2-D code predictions.

\section{CFD-ACE+/Fluent Results vs. Some Literature Results}

\subsection{Surface Heat Flux and Temperature Difference}

Experimental and numerical heat exchanger surface heat flux and temperature difference between the gas temperature at the radial center of the heat exchanger and the heat exchanger wall at $1 / 16,1 / 8,1 / 4,1 / 2$ of the heat exchanger length from the entrance and the "end" of the heat exchanger opposite the entrance are plotted against the crank angle starting at BDC. The surface heat flux data are illustrated in Figs. 13(a-d) and the corresponding temperature difference data are illustrated in Figs. 14(a-d). Kornhauser's experimental results ${ }^{20}$, Figs. 13(a) and 14(a), Tew's modified CAST code results ${ }^{36}$, Figs. 13(b) and 14(b), CFD-ACE+ results of the 2-space model ${ }^{12}$, Figs. 13(c) and 14(c) and Fluent results of the present study of the 3-space model, Figs. 13(d) and 14(d) are presented and compared. Note that the sign of the heat transfer value generated by the modified CAST (Fig. 13(b)) is opposite to that of Kornhauser's (Fig. 13(a)). This is because in the numerical codes (CAST, CFD-ACE+ and Fluent), heat transfer is defined to be positive for heat flow from the wall to the gas (opposite to Kornhauser's definition). The signs of the heat transfer values generated by CFD-ACE+ (Fig. 13(c)) and Fluent results (Fig. 13(d)) were reversed in order to have the vertical coordinates appear like Kornhauser's plot. The experimental (Kornhauser's) and numerical (CAST, CFD-ACE+ and Fluent) results for the heat flux and temperature difference are generally in good qualitative agreement but with some notable differences. The variations in the numerical results appear smoother than Kornhauser's results. The magnitudes of the heat flux and temperature difference are also somewhat different. At maximum compression the highest values for the heat flux and temperature difference are observed at the 1/16 and 1/8 location respectively for the experimental results and at the entrance of the heat exchanger for the numerical results. The heat flux and temperature difference values are smallest at the 1/8 position (Kornhauser's and Tew's), at the end of the heat exchanger (2-space model) and at the $1 / 2$ position (3-space model). During the expansion phase $\left(180^{\circ} \leq \theta \leq 360^{\circ}\right)$ when flow is from the heat exchanger to the cylinder, profiles of the heat flux and temperature difference generated by the CAST code are most insensitive to location along the heat exchanger surface. Kornhauser's results show significant profile differentiation for both the heat flux and temperature difference in this crank angle range. The minimum values are insensitive to position along the heat exchanger surface for the modified CAST code. There are also noticeable differences in the magnitudes of the heat flux and temperature difference reported by the codes at the heat exchanger entrance and end.

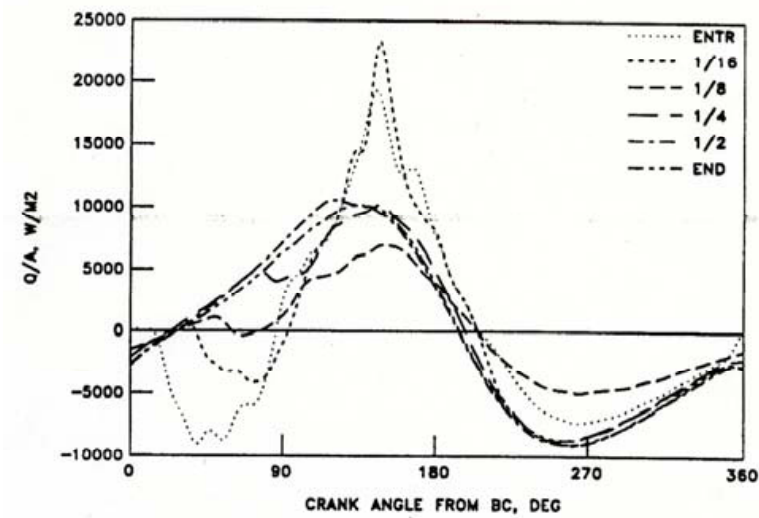

Figure 13(a). Heat flux vs. Crank angle at various positions along heat exchanger surface relative to entrance to cylinder. (Kornhauser's Experimental Data ${ }^{3}$ : Run \#10271539, 201.7 RPM, $1.008 \mathrm{MPa}$ (arithmetic mean pressure), $\mathrm{T}_{\text {wall }}=294 \mathrm{~K}$ ).

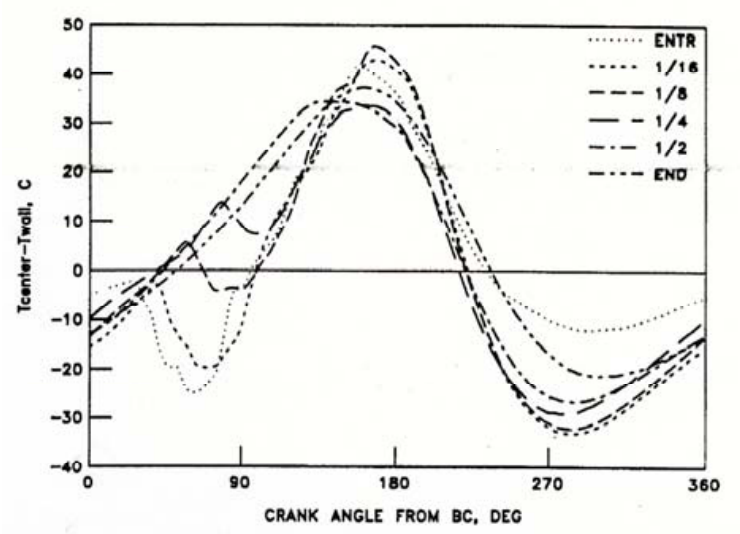

Figure 14(a). Temp. difference $\left(\mathrm{T}_{\text {center }}-\mathrm{T}_{\text {wall }}\right)$ vs. Crank angle at various positions along heat exchanger surface relative to entrance to cylinder. (Kornhauser's Exptal Data ${ }^{3}$ : Run \#10271539, 201.7 RPM,1.008 MPa (arithmetic mean pressure), $\mathrm{T}_{\text {wall }}=294 \mathrm{~K}$ ). 


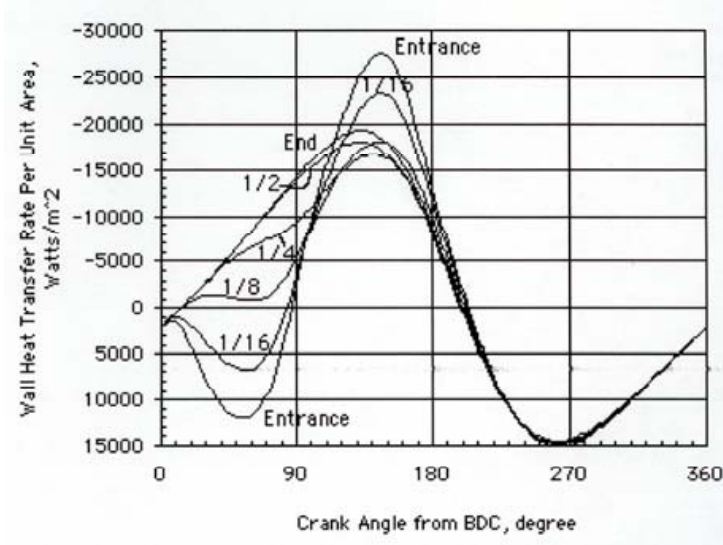

Figure 13(b). Heat flux vs. Crank angle at various positions along heat exchanger surface relative to entrance to cylinder. (Tew's Modified Cast Code Calculations ${ }^{70^{*}}: 34 \mathrm{x}$ 20 grids, 120 tspc.).

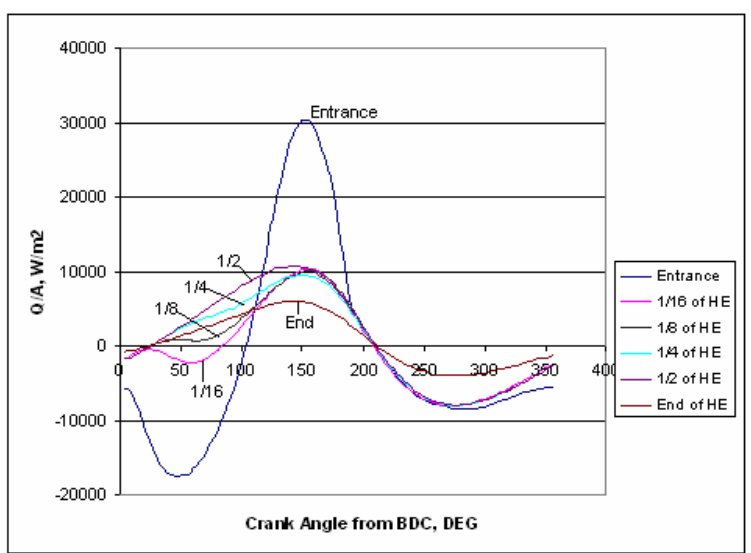

Figure 13(c). Heat flux vs. Crank angle at various positions along heat exchanger surface relative to entrance to cylinder. (CFD-ACE+ Code Calculations ${ }^{19}: 34 \times 20$ grids, 120 tspc.)

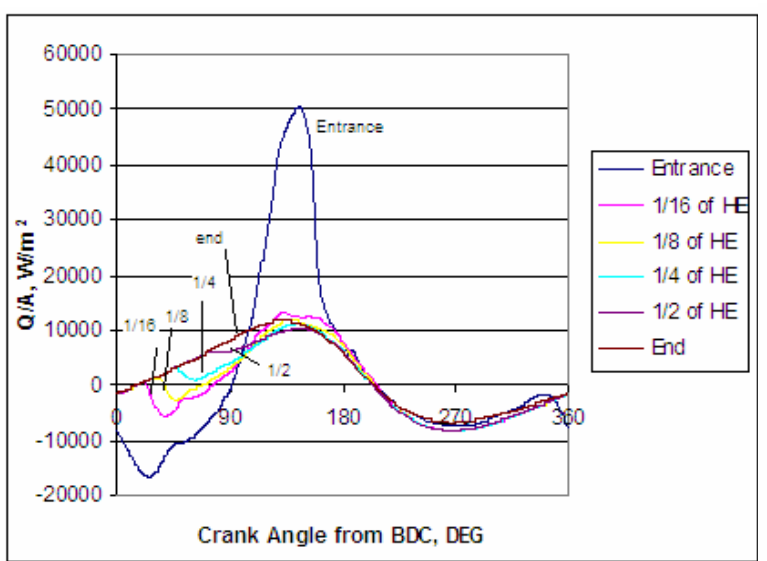

Figure 13(d). Heat flux vs. Crank angle at various positions along heat exchanger surface relative to entrance to cylinder. (Fluent Code Calculations: 34 x 20 grids, 120 tspc).

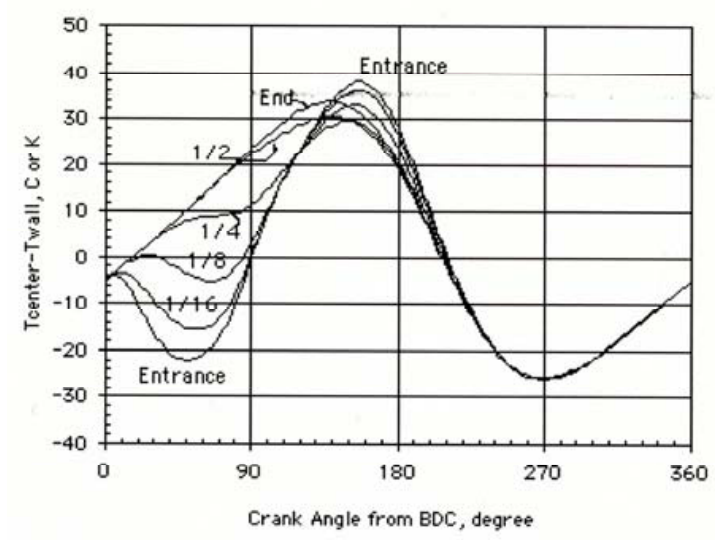

Figure 14(b). Temp. difference $\left(T_{\text {center }}-T_{\text {wall }}\right)$ vs. Crank angle at various positions along heat exchanger surface relative to entrance to cylinder. (Tew's Modified Cast Code Calculations $\mathrm{s}^{70^{*}}: 34$ x 20 grids, 120 tspc.).

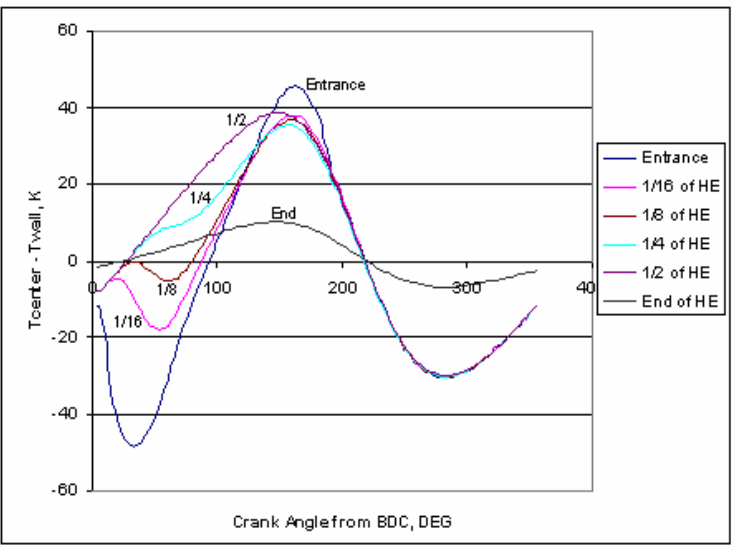

Figure 14(c). Temp. difference $\left(\mathrm{T}_{\text {center }}-\mathrm{T}_{\text {wall }}\right)$ vs. Crank angle at various positions along heat exchanger surface relative to entrance to cylinder. (CFD-ACE+ Code Calculations ${ }^{19}: 34$ x 20 grids, 120 tspc.)

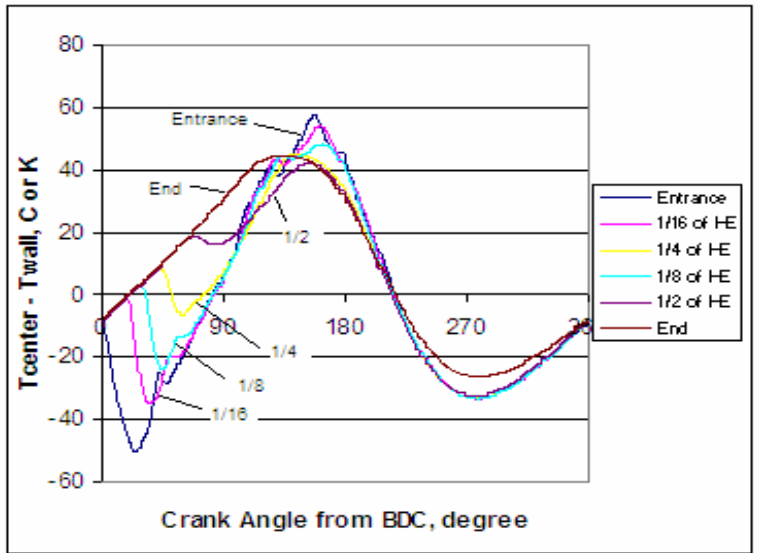

Figure 14(d). Temp. difference $\left(\mathrm{T}_{\text {center }}-\mathrm{T}_{\text {wall }}\right)$ vs. Crank angle at various positions along heat exchanger surface relative to entrance to cylinder. (Fluent Code Calculations: $34 \times 20$ grids, 120 tspc). 
The effect of the regenerator is clearly noticed at the entrance of the heat exchanger where the maximum values of the heat flux and temperature difference are elevated.

\subsection{Temperature Contours}

Temperature contour plots at $90^{\circ}$ before TDC where maximum piston velocity is toward the heat exchanger are illustrated in Figs. 15(a-e). Similar qualitative results are obtained in the heat exchanger and cylinder spaces using the CFD-ACE+ code (Figs. 15(a-c)), the modified CAST code (Fig. 15(d)) and the Fluent code (Fig. 15(e)). It is observed that whereas in the heat exchanger space the fluid temperature increases from the wall region towards the interior (Fig. 15(b-e)), in the cylinder space the opposite trend is observed with fluid temperature decreasing as one moves into the cylinder interior from the wall region (Fig. 15(a,d,e)). Also, the cooler fluid is at the entrance of the heat exchanger and the fluid becomes warmer as one moves towards the end of the heat exchanger (Fig.15(b,d,e)). Notice that unlike in the 2-space and 3-space models (Figs. 15(a,e)), the outer cylinder wall is flush with the outer wall of the heat exchanger in the CAST model (Fig. 15(d)). Due to this difference in geometries, it is observed that the higher temperature contours are situated close to the upper cylinder region in the 2-space and 3-space models and not at the entrance to the heat exchanger as in CAST.

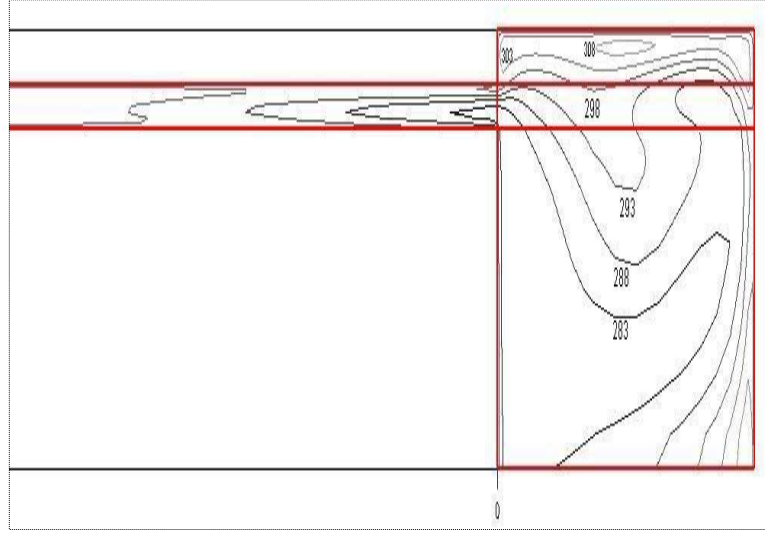

Figure 15(a). Temperature contours for cylinder and entrance of HXer. (CFD-ACE+, 147x51grids, $\Delta \mathrm{t}=$ 6.19E-04, (480 tspc); $90^{\circ}$ before TDC; Op. Conds.: 201.7 RPM. 1.008 MPa)

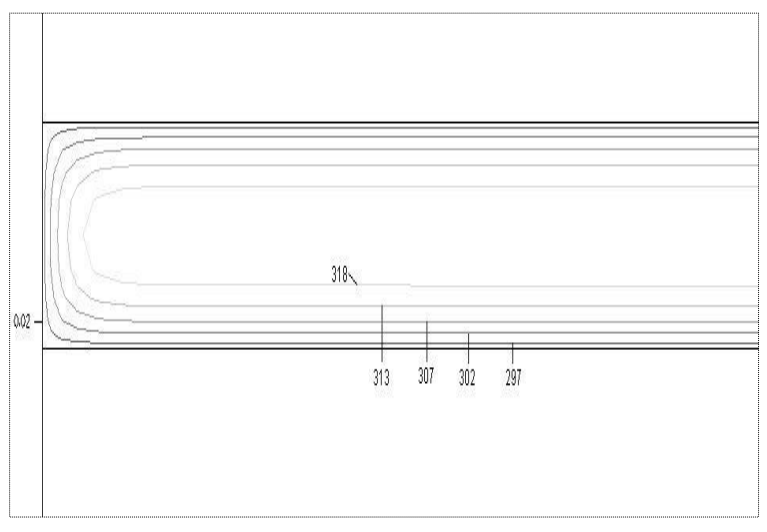

Figure 15(c). Zoom in on temperature contours for end of HXer. (CFD-ACE+, 147x51 grids, $\Delta \mathrm{t}=6.19 \mathrm{E}-04$, (480 tspc); $90^{\circ}$ before TDC; Op. Conds.: 201.7 RPM, $1.008 \mathrm{MPa})$

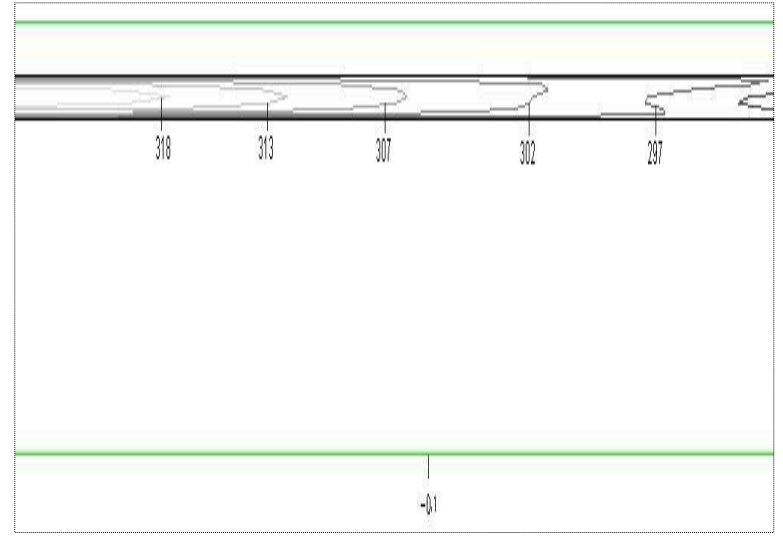

Figure 15(b). Temperature contours for middle part of heat exchanger. (CFD-ACE,$+ 147 \times 51$ grids, $\Delta \mathrm{t}=$ 6.19E-04, (480 tspc); $90^{\circ}$ before TDC; Op. Conds.: 201.7 RPM. 1.008 MPa)

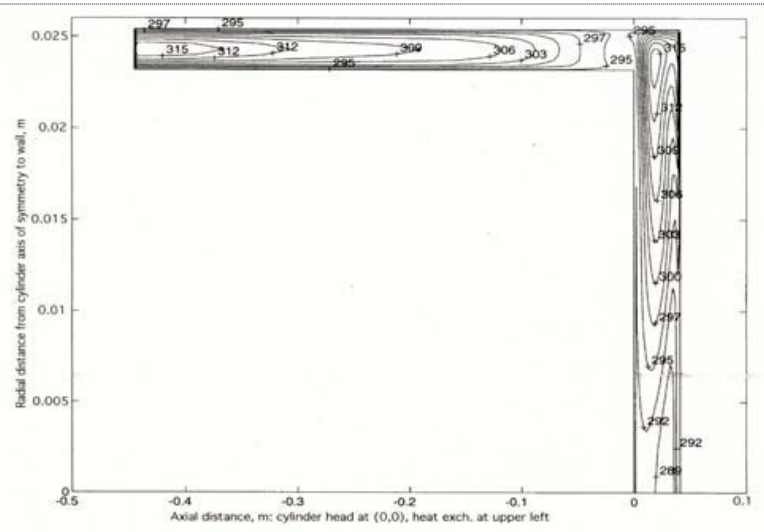

Figure 15(d). Temperature contours for entire domain (Modified CAST Run \#13 with 82x20 grids, 960 tspc. Simulation of Kornhauser's [3] Experimental Run \#10271539. Maximum Piston Velocity toward HXer $90^{\circ}$ before TDC. $)$. 


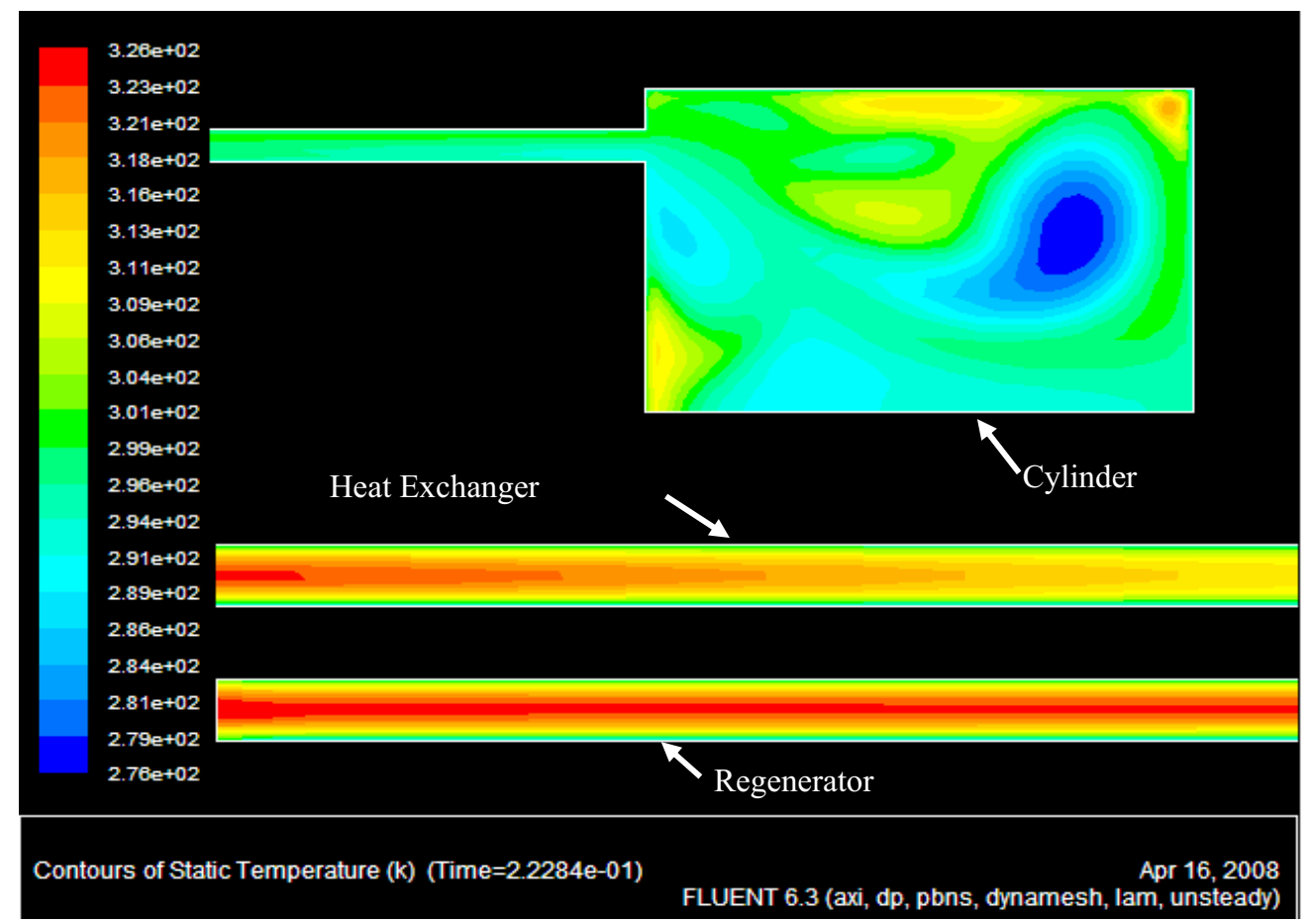

Figure : 15(e) Temperature contours of 3-space domain

(Fluent, 147x46 grids $\Delta \mathrm{t}=6.19 \mathrm{E}-04$, (480 tspc); $90^{\circ}$ befor TDC; Op. Conds.: 201.7 RPM, 1.008 MPa)

As in the heat exchanger space, the fluid temperature in the regenerator increases from the wall region towards the interior.

\subsection{Velocity Vectors}

Figures 16(a-e) show laminar velocity vector field plots in the 2-space model (Figs. 16(a-d)) and 3-space model (Fig. 16(e)). The CAST code (Fig. 16(b)), the CFD-ACE+ code (Fig. 16(c)) and the Fluent code (Fig. 16(e)) show the fluid accelerating around the corner of the inner wall of the annulus to enter the heat exchanger. The velocity is primarily radial along the cylinder head approaching the heat exchanger entrance. Across the heat exchanger entrance, as the fluid prepares to turn the corner, the velocity changes from mostly radial at the lower "corner" to mostly axial at the upper wall for the CAST model. For the CFD-ACE+ and Fluent models, because of the slight difference in geometries, the transition from radial to axial flow takes place at the lower and upper corners of the heat exchanger entrance. Mass conservation requires a substantial increase in axial velocity near the outer wall for the CAST model (Fig. 16(b)) or in the middle, away from the walls for the CFD-ACE+ (Fig. 16(c)) and Fluent (Fig. 16(e)) models, as the fluid enters the exchanger. Then the fluid begins to redistribute across the annulus and the axial velocity near the outer wall (CAST model) or near the inner and outer walls (CFD-ACE+ and Fluent models) decreases.

As with the CAST results (Fig. 16(a)), the CFD-ACE+ results (Fig. 16(d)) show that the velocity tends to zero at the dead end of the heat exchanger in the 2-space model and Fluent results (Fig.16(f)) show a reduction in the velocity field as the fluid moves toward the regenerator entrance. 

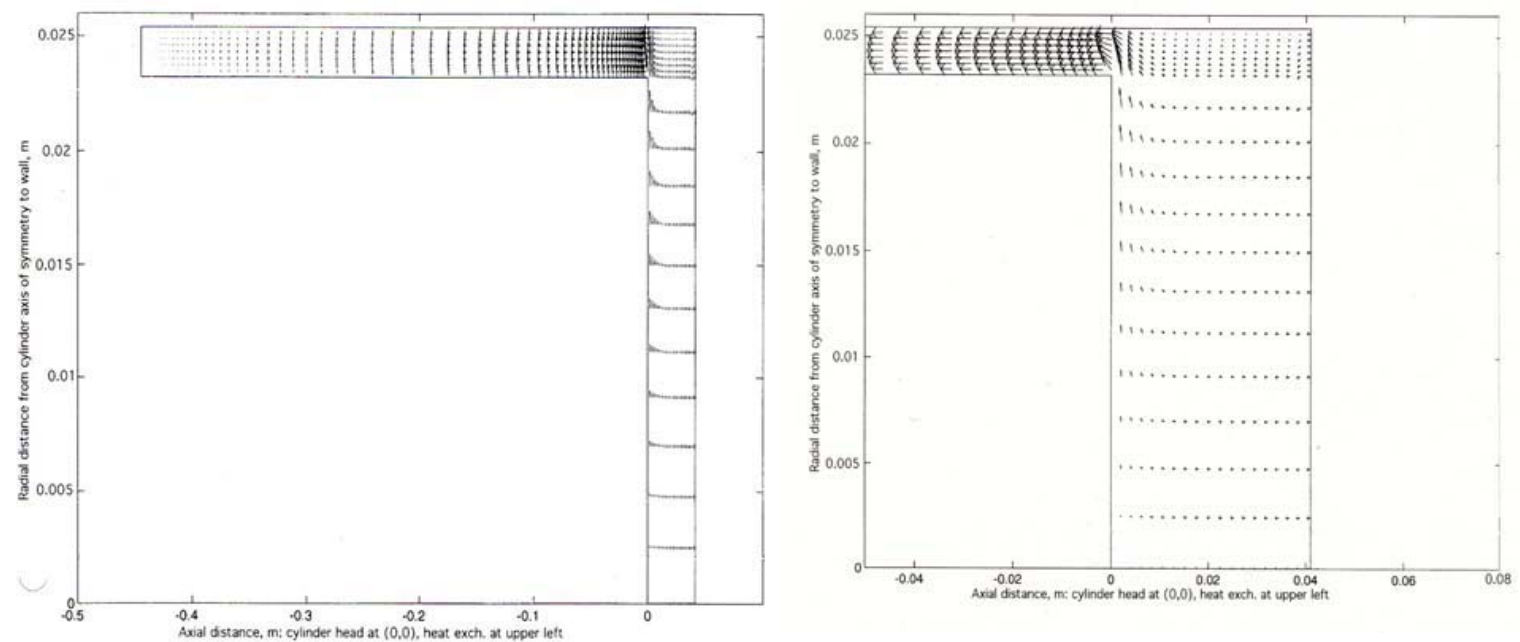

Figure 16(a). Velocity field plot for entire domain (Modified CAST Run \#13 with 82x20 grids, 960 tspc. Simulation of Kornhauser's [3] Experimental Run \#10271539. Maximum piston velocity toward HXer.).

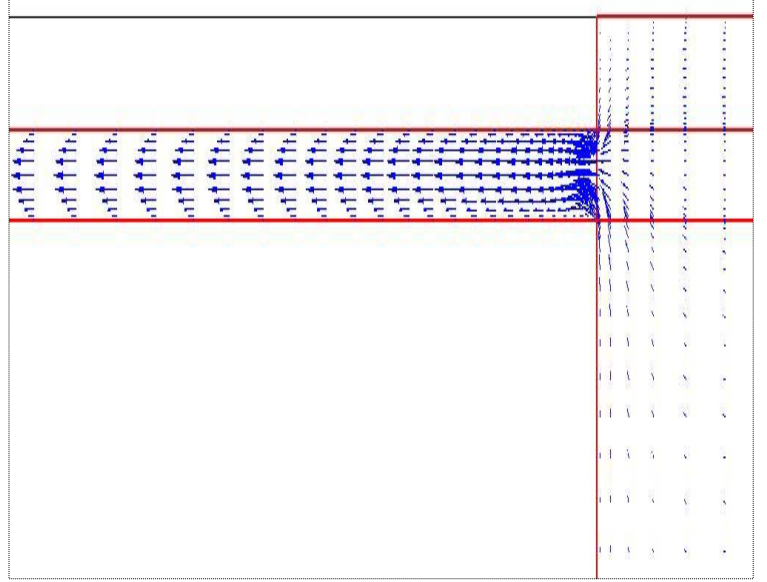

Figure 16(c). Zoom in on velocity field plot at HXer entrance. (CFD-ACE+, Grid $=147 \times 51, \Delta t=6.19 \mathrm{E}-04$, ( 480 tspc); Maximum piston velocity toward the $90^{\circ}$ before TDC; Operating Conditions: 201.7 RPM, 1.008 $\mathrm{MPa}$ )
Figure 16(b). Zoom in on velocity field plot at HXer entrance (Modified CAST Run \#13 with 82x20 grids, 960 tspc. Simulation of Kornhauser's [3] Experimental Run \#10271539. Maximum piston velocity toward HXer.).

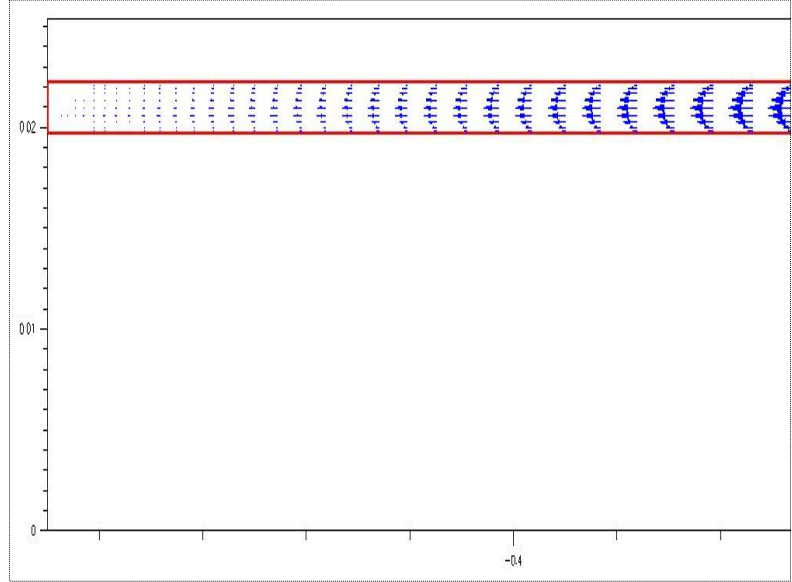

Figure 16(d). Zoom in on velocity field plot at HXer end. (CFD-ACE+, Grid $=147 \times 51, \Delta t=6.19 \mathrm{E}-04,(480$ tspc); Maximum piston velocity toward the $90^{\circ}$ before TDC; Operating Conditions: 201.7 RPM, 1.008 MPa.) 


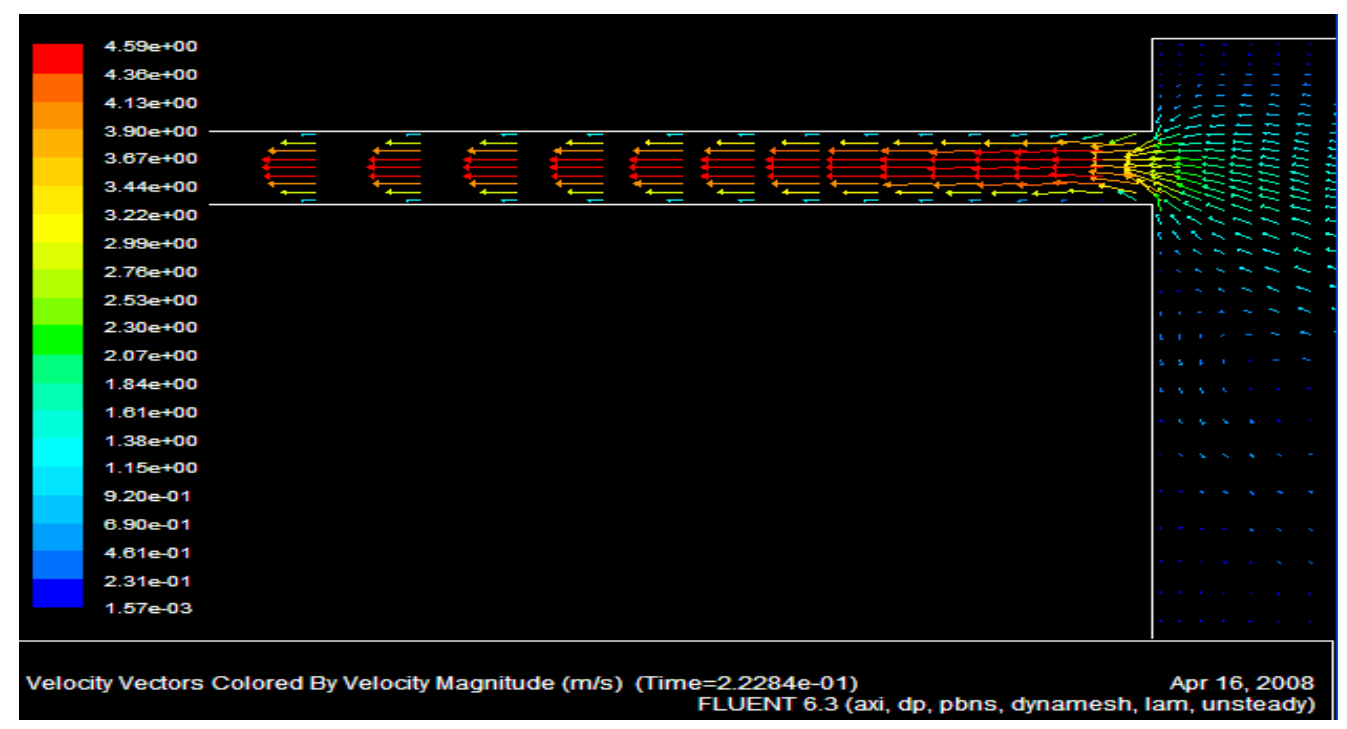

Figure 16 (e) Zoom in on velocity field plot at HXer entrance. Fluent Grid $=147 \times 56, \Delta t=6.19 \mathrm{E}-04$, (480 tspc); Maximum piston velocity toward the $90^{\circ}$ before TDC; Operating Maximum piston velocity toward the $90^{\circ}$ before TDC; Operating Conditions: 201.7 RPM, 1.008 MPa).

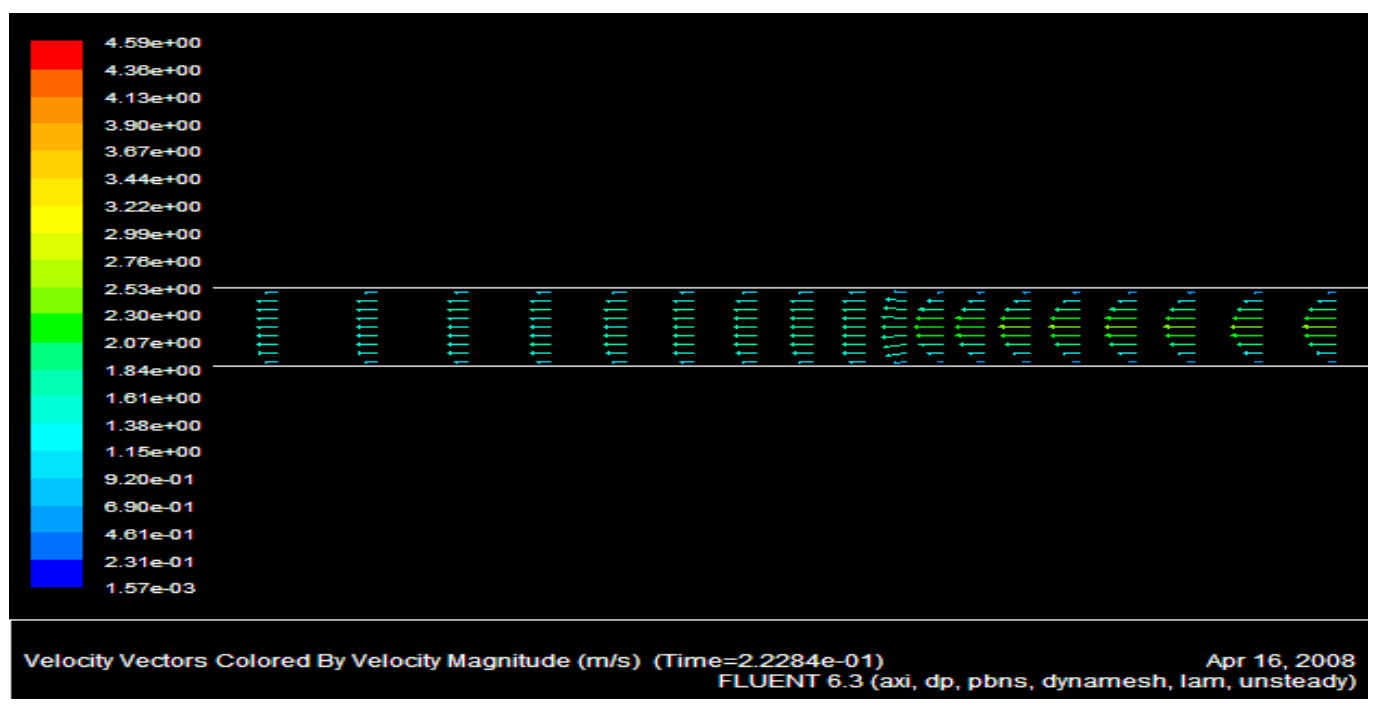

Figure 16 (f) Zoom in on velocity field plot at HXer end. Fluent Grid $=147 \times 56$, $\Delta \mathrm{t}=6.19 \mathrm{E}-04,(480 \mathrm{tspc})$; Maximum piston velocity toward the $90^{\circ}$ before TDC;

Operating Maximum piston velocity toward the $90^{\circ}$ before TDC; Operating Conditions: $201.7 \mathrm{RPM}, 1.008 \mathrm{MPa}$ ). 


\section{Thermodynamic Loss Post Processing}

Equations (38-43) clearly show that local entropy production depends functionally on the local values of heat transfer rate, temperature, pressure, density, mass-specific entropy, velocity and viscous dissipation. Thus entropy generation can be considered a derived quantity that can be computed by post-processing experimental or numerical flow fields. Equation (44) shows that local entropy production is also a function of availability energy loss and temperature.

\section{Using Sage}

Sage entropy generation results (external and internal) for the 3-space domain are calculated from Eq. (44) using availability loss result components obtained from Sage output file viz.:

$$
\dot{\mathrm{S}}_{\mathrm{gen}}=\frac{\text { Availability Energy Loss }}{\mathrm{T}_{\mathrm{L}}}=\frac{\text { AEfric }+ \text { AEQw }+ \text { AEQx } \pm \mid \text { AEDiscr } \mid}{\mathrm{T}_{0}}
$$

The parameters AEfric, $\mathrm{AEQw}$, and $\mathrm{AEQx}$ are available energy losses due to flow friction, surface heat flow and axial heat flow respectively. |AEDiscr| is the absolute value of the discrepancy between the total available energy loss due to internal entropy generation (AEinternal $=\mathrm{AEfric}+\mathrm{AEQw}+\mathrm{AEQx})$ and that due to external entropy generation, AEexternal. The "+" is used when it is assumed that AEexternal is greater than AEinternal and the ""sign is used when the reverse is the case. These assumptions are arbitrary since the relative magnitudes of AEexternal and AEinternal cannot be determined apriori. A discrepancy of zero implies the total available energy loss due to internal entropy generation is equal to that due to external entropy generation.

\section{Using Fluent}

2.1 External Entropy Generation, $\dot{\mathrm{S}}_{\text {gen (ext.) }}$

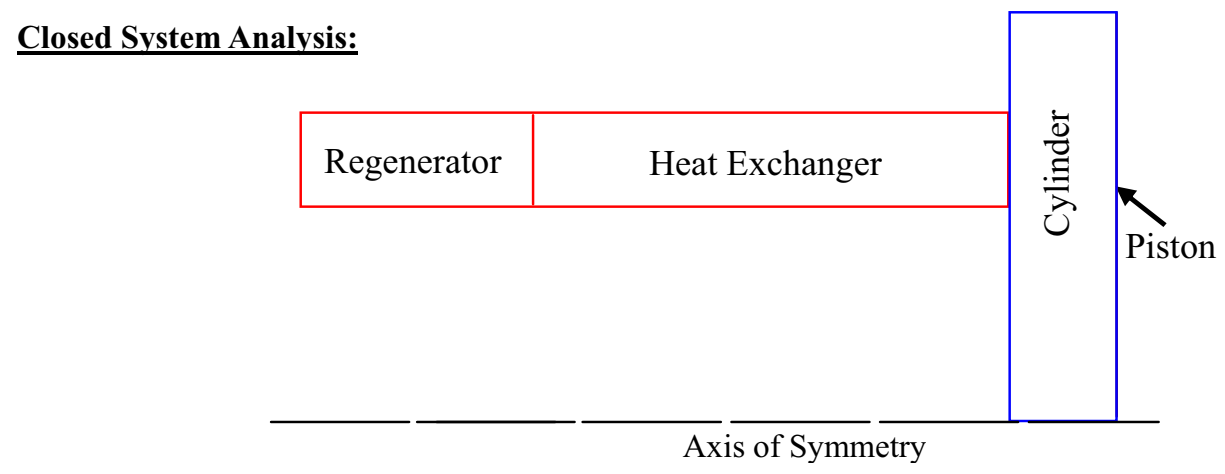

Figure 17. 3-space Model Set-Up for External Entropy Generation Analysis (Closed System)

The 3-space model shown in Fig. 17 above taken as a whole constitutes a closed reciprocating system. When viewed as a closed system for external entropy generation analysis, Eq. (38) for the non-porous domain and Eq.(39) for the porous domain are thus reduced to:

$$
\begin{array}{ll}
\text { Non-porous domain: } & \dot{\mathrm{S}}_{\substack{\text { gen, sys, cyl(ext) } \\
\text { (non-porous) }}}=\oint_{\text {eriod }} \int_{d \mathrm{~s}}\left(\frac{\overrightarrow{\mathrm{q}}_{\alpha}}{\mathrm{T}_{\alpha}} \cdot \hat{\mathrm{n}}_{\alpha}+\frac{\overrightarrow{\mathrm{q}}_{\beta}}{\mathrm{T}_{\beta}} \cdot \hat{\mathrm{n}}_{\beta}\right) \mathrm{dAdt} \geq 0 \\
\text { Porous domain: } \quad\left\langle\dot{\mathrm{S}}_{\substack{\text { gen, sys, cyl(ext) } \\
\text { porous }}}\right\rangle=\oint_{\text {period }} \int_{\mathrm{ss}}\left(\varepsilon_{\alpha} \mathrm{N}_{\mathrm{k}} \frac{\left\langle\overrightarrow{\mathrm{q}}_{\alpha}\right\rangle^{\alpha}}{\left\langle\mathrm{T}_{\alpha}\right\rangle^{\alpha}} \cdot \hat{\mathrm{n}}_{\alpha}+\frac{\left\langle\overrightarrow{\mathrm{q}}_{\beta}\right\rangle^{\beta}}{\left\langle\mathrm{T}_{\beta}\right\rangle^{\beta}} \cdot \hat{\mathrm{n}}_{\beta}\right) \mathrm{dAdt} \geq 0
\end{array}
$$


in which case only the results of surface heat transfer and temperature for both the fluid and solid phases are postprocessed for external entropy generation. Eq. (52) is used to integrate the heat transfer along the isothermal bounding surfaces of the cylinder and heat exchanger and Eq. (53) is used to integrate the heat transfer along the adiabatic bounding surfaces of the regenerator.

\section{Open System Analysis:}

The 3-space model can also be analyzed, by considering its separate components - regenerator, heat exchanger space and cylinder space - as open systems for which both entropy transfer components (heat and mass flow) of Eqs. (38) and (39) are now applicable. In order to simplify the analysis, the 3-space model was partitioned into five sub-domains - outer, mid and inner sub-domains in the cylinder space plus the heat exchanger and regenerator subdomains as shown in Fig. 18 below. Fluent generated results of surface heat transfer, temperature, mass flow rate and mass specific entropy are post-processed for external entropy generation.

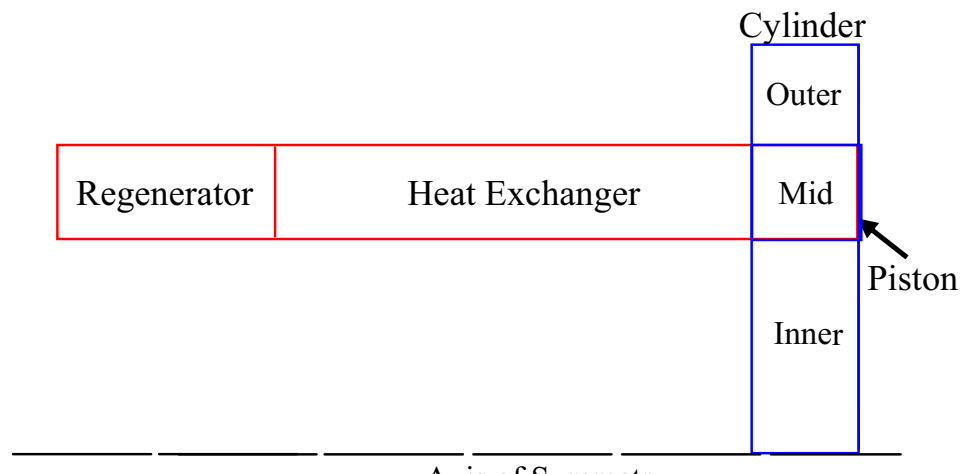

Axis of Symmetry

Figure 18. 3-space Model Set-Up for External Entropy Generation Analysis (Open System)

The external entropy generation is calculated for each sub-domain with the sum of the results for the separate sub-domains yielding the total external entropy generation for the 3-space model. The result for this open system analysis should equal the result for the closed system analysis.

\section{User Defined Functions}

User defined functions (UDFs) can be used to post-process any Fluent generated closed or open system data surface heat transfer, temperature, velocity gradients, mass flow and mass specific entropy. UDFs are written in FORTRAN 90 and interfaced with the Fluent solver. The summation of surface, volume and time values, implied by the integrals in Eqs. (38-40 and 43) is facilitated with the use of do loops in the UDFs. In order to minimize program complexity, the UDFs are written to sum up the surface and volume integral values at each time step only and thereafter data is exported to an Excel spreadsheet for calculation of the cyclic time integral values. Carefully constructed algorithms are needed for implementation of Eqs. (38-40 and 43). These algorithms constitute the UDFs.

For the closed system analysis, integrated surface heat transfer rates generated each time step are exported to an Excel spreadsheet for calculation of the cyclic time integral value. Division of the cyclic integral value by the constant surface temperature completes the calculation of the external entropy generation.

\subsection{Internal Entropy Generation, $\dot{\mathrm{S}}_{\mathrm{gen} \text { (int.). }}$}

The sub-domains in the model may be further refined for calculation of internal entropy generation as shown in Figure 19, below, in order to obtain a better resolution of the losses. Internal entropy generation due to conductive heat flow and viscous dissipation is calculated for the non-porous region using Eq. (40) and internal entropy generation due to conductive heat flow, film heat transfer, viscous and inertial losses for the porous region using Eq. (43). Results of heat transfer, temperature and gradients of temperature, velocity and gradients of pressure are postprocessed via UDFs written for each sub-domain in the 3-space model.

With the entropy generation known, the availability energy loss can be calculated using Eq. (44). Local and global distribution of entropy generation rates due to the various system irreversibilities can be evaluated via a histogram map. 


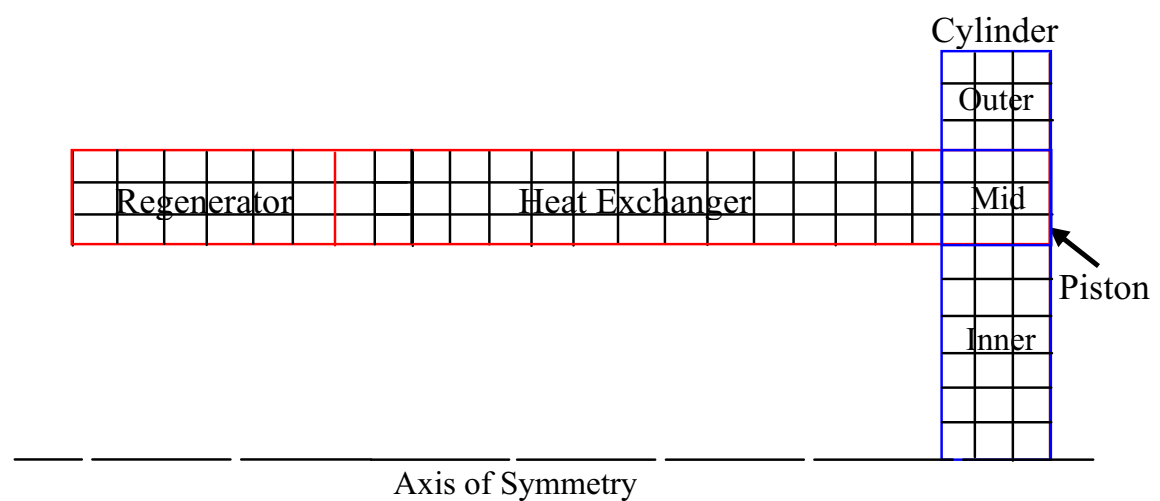

Figure 19. 3-space Model Set-Up for Internal Entropy Generation Analysis

\section{Entropy Generation and Availability Loss Results}

The results of the post-processing analysis are shown and discussed below. Figures 20 and 21 below illustrate the results of the external- and internal-entropy generations for each of the sub-domains in the 2-space and 3-space models. Figs. 20(a,c) show the functional dependence of entropy generation on the number of cycles of piston motion and Figs. 20(b,d) show histograms of the entropy generation values characterizing the contributions of each sub-domain to the external entropy generation. Figures $21(\mathrm{a}-\mathrm{d})$ illustrate corresponding results for the internal entropy generation.

Figs. 20(a,c) show the external entropy generation plots to be independent of the number of cycles of piston motion beyond the third cycle. The negative values for the external entropy generation in the cylinder sub-domains (Figs. 20(a-d)) should be interpreted as the cylinder acting as entropy sink. That is, these sub-domains appear to extract entropy from their surroundings (due to heat entering the cylinder). Because of the cylinder acting as entropy sink, the heat exchanger's contribution exceeds the total external entropy generation by about $81 \%$ in the 2 -space model (Fig. 20(b)) and by about 181\% in the 3-space model (Fig. 20(d)). Figs. 21(a,c) show the internal-entropy generation plots to be independent of the number of cycles of piston motion one cycle earlier than does the external entropy generation plots. The heat exchanger's contribution to the total internal-entropy generation falls short of the total by about $14 \%$ and $28 \%$ in the 2 -space (Fig. 21(b)) and 3-space (Fig. 21(d)) models respectively. In this case, the cylinder sub domains contribute positively to the total entropy generation.

Of the three cylinder's sub-domains, the outer cylinder's contribution to the entropy generation is most significant in the 2-space model (external or internal) and 3-space model (internal). The inner cylinder's contribution to the external entropy generation is most significant in the 3-space model. The mid-cylinder's and regenerator's contributions to the external and internal entropy generations are minimal or negligible where relevant. When taken as a whole, the cylinder's and regenerator's contributions to both external and internal entropy generations are minimal compared to the heat exchanger's contribution. 


\section{External Entropy Generation from 2-space Sub-domains (CFD-ACE+)}

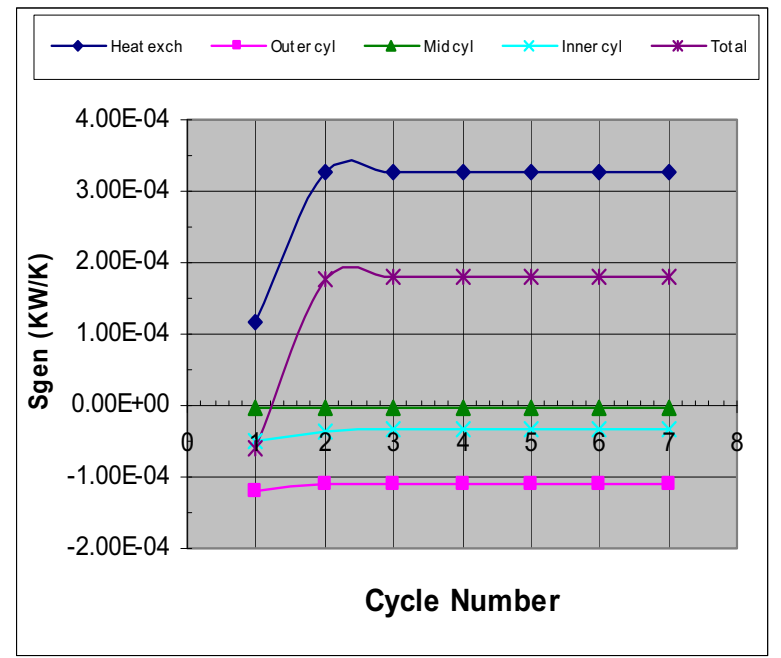

Figure 20(a). 2-space External Entropy generation vs. Cycle No. $\left(201.7 \mathrm{RPM}, 1.008 \mathrm{MPa}\right.$., $\mathrm{T}_{\text {wall }}=294 \mathrm{~K} ;$ Grid size $=147 \mathrm{x} 46$, \#tspc $\left.=480\right)$.

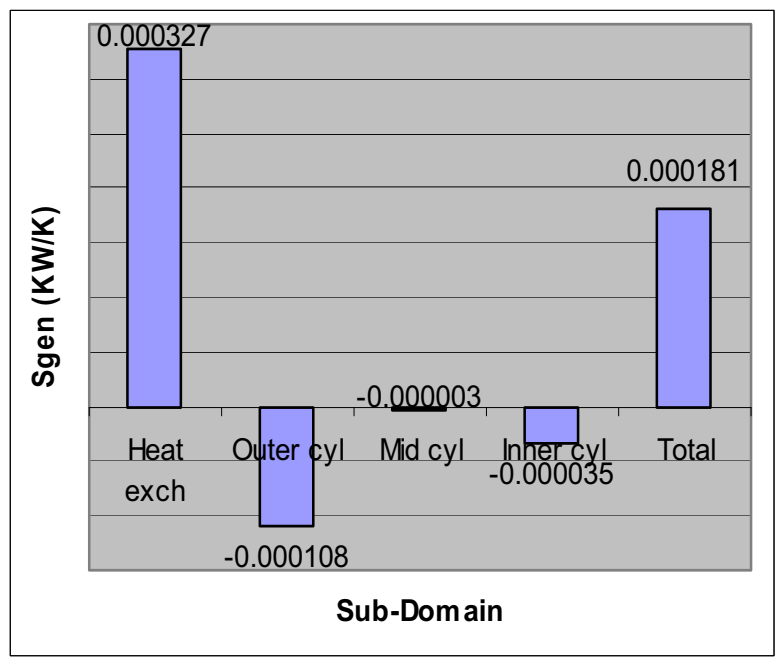

Figure 20(b). 2-space External Entropy generation vs. Region $\left(201.7 \mathrm{RPM}, 1.008 \mathrm{MPa}\right.$., $\mathrm{T}_{\text {wall }}=294 \mathrm{~K}$; Grid size $=147$ x 46, \#tspc $=480$ )

External Entropy generation from 3-space Sub-domains (Fluent)

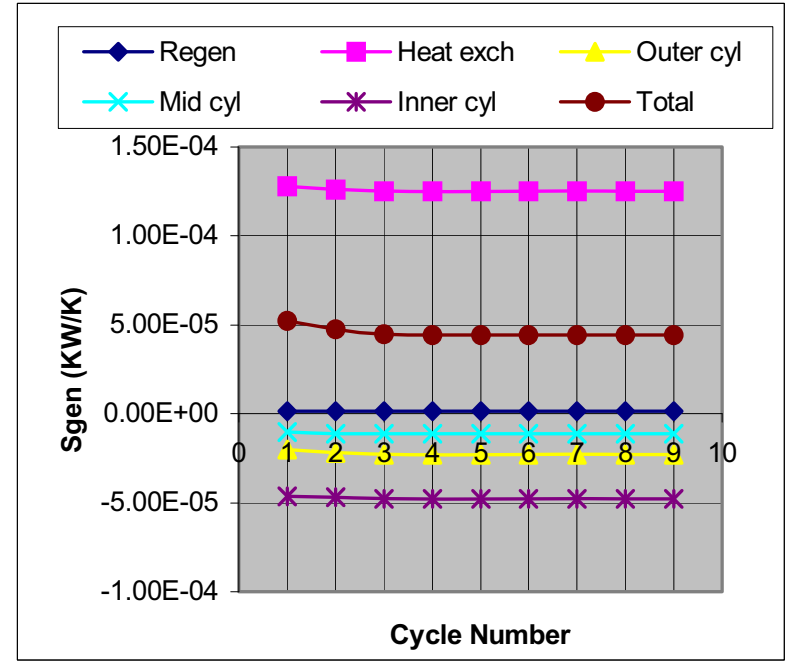

Figure 20(c). 3-space External Entropy generation vs. Cycle No. (201.7 RPM, 1.008 MPa., $\mathrm{T}_{\text {wall }}=294 \mathrm{~K} ;$ Grid size $=147$ x 46, \#tspc $=480$ ).

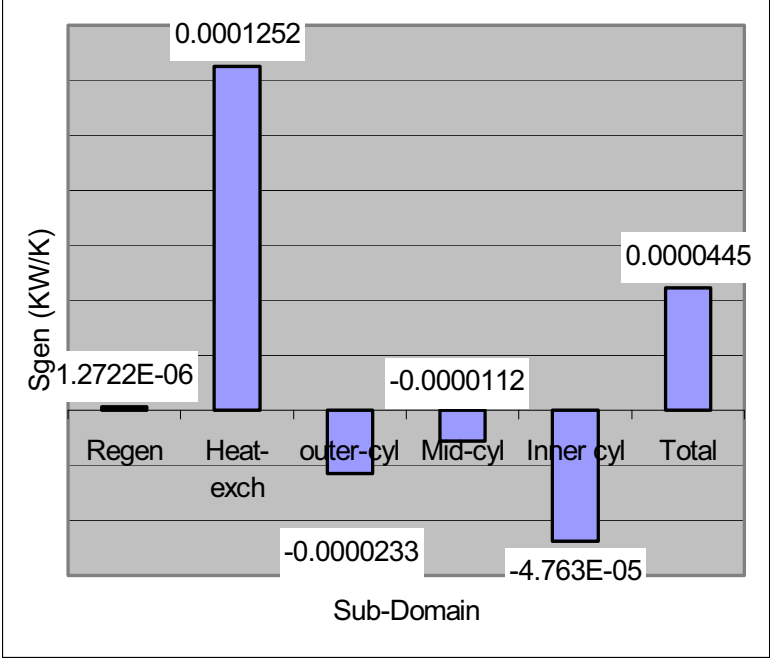

Figure 20(d). 3-space External Entropy generation vs. Region (201.7 RPM, 1.008 MPa., $\mathrm{T}_{\text {wall }}=294 \mathrm{~K} ;$ Grid size $=147 \mathrm{x} 46$, \#tspc $=480$ ). 
Internal Entropy Generation in 2-space Sub-domains (CFD-ACE+)

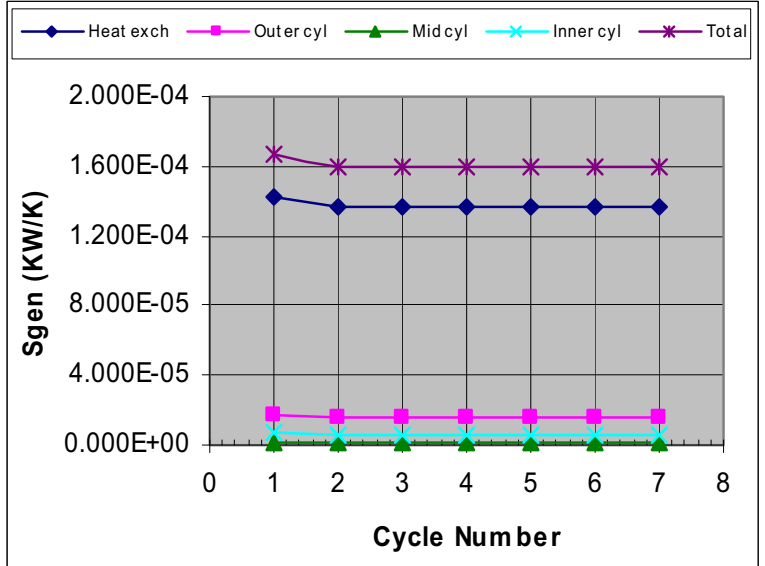

Figure 21(a). 2-space Internal Entropy generation vs. Cycle No. (201.7 RPM, 1.008 MPa., $\mathrm{T}_{\text {wall }}=294 \mathrm{~K} ;$ Grid size $=147$ x 46, \#tspc $\left.=480\right)$

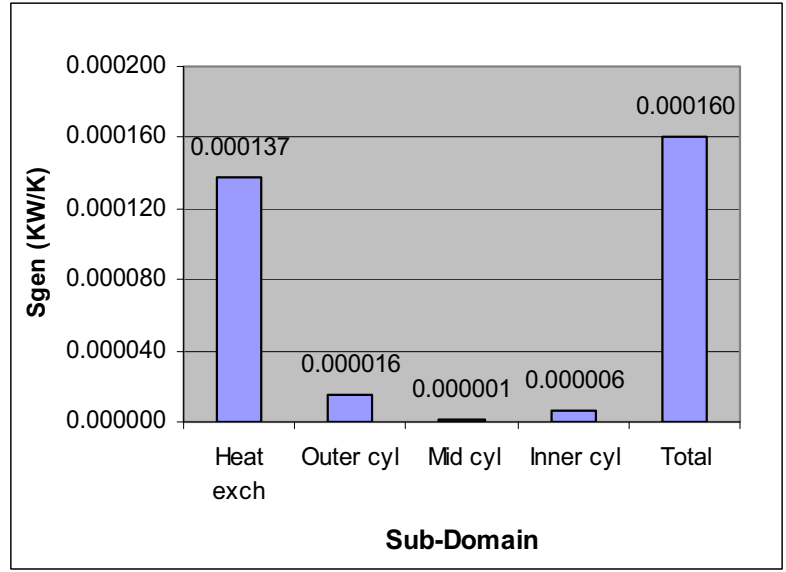

Figure 21(b). 2-space Internal Entropy generation vs. Region $\left(201.7 \mathrm{RPM}, 1.008 \mathrm{MPa}\right.$., $\mathrm{T}_{\text {wall }}=294 \mathrm{~K} ;$ Grid size $=147$ x 46, \#tspc $\left.=480\right)$.

Internal Entropy Generation in 3-space Sub-domains (Fluent)

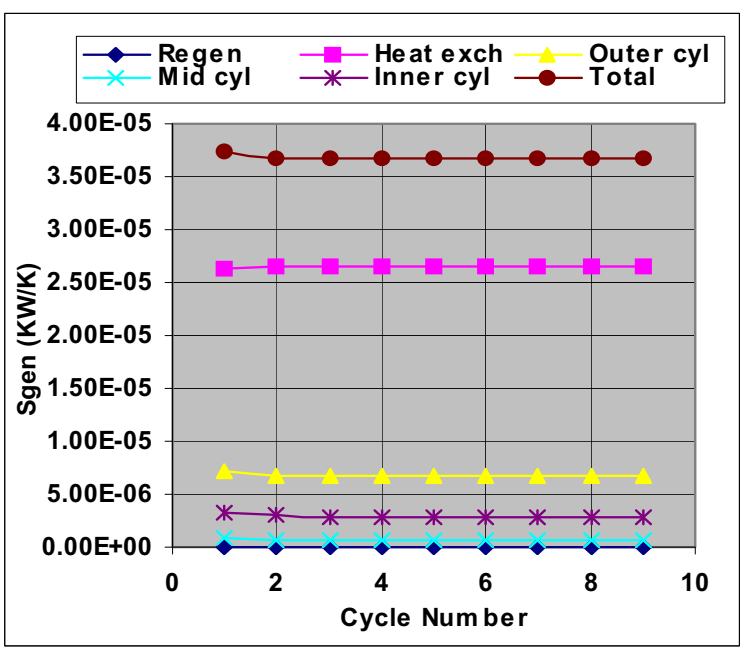

Figure 21(c). 3-space Internal Entropy generation vs. Cycle No. (201.7 RPM, 1.008 MPa., $\mathrm{T}_{\text {wall }}=294 \mathrm{~K} ;$ Grid size $=147$ x 46, \#tspc $\left.=480\right)$.

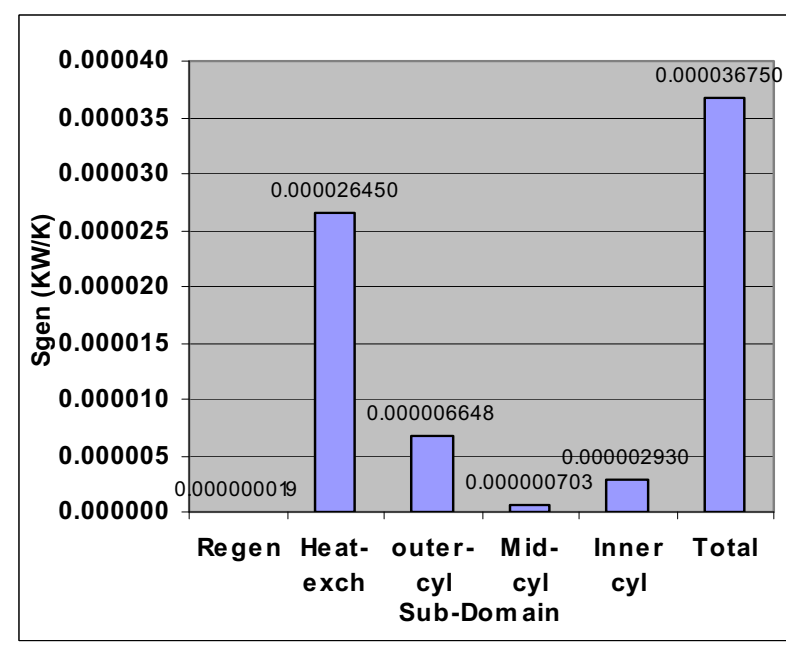

Figure 21(d). 3-space Internal Entropy generation vs. Region $\left(201.7 \mathrm{RPM}, 1.008 \mathrm{MPa}\right.$., $\mathrm{T}_{\text {wall }}=294 \mathrm{~K} ;$ Grid size $=147$ x 46, \#tspc $\left.=480\right)$.

A clear inference from these plots is that the major sub-domain contribution to entropy generation is from the heat exchanger. The heat exchanger is observed to contribute more to the total external entropy generation than it does to the total internal entropy generation.

Figure 22 shows the distribution of entropy generation inside the 2-space and 3-space models using Fluent. The impact of the entropy generation due to conductive heat transfer, fluid friction and mass transfer on the efficiency of the models is quantified. Under the specified condition (201.7 RPM, 1.008 MPa., $\mathrm{T}_{\text {wall }}=294$ ), relatively higher losses are experienced in the heat exchanger than in other sub-components of each model. This is followed by losses in the outer cylinder and inner cylinder. Contributions from the mid-cylinder are very negligible. The heat exchanger's conductive heat transfer has the most impact on the model efficiency followed by outer and inner cylinder conductive heat transfer. Contributions to the entropy generation due to viscous dissipation, mass transfer and conductive heat transfer are very negligible. Please note that in Fig. 22(d), under the "Total Volume" column, "Viscous" has been mislabeled "Conductive" and vice versa. The relatively high losses in the heat exchanger support the extensive effort in the Stirling community to optimize the regenerator which is a form of heat exchanger. 
In compact-heat-exchanger passages for example, improvements can be made in the constructive details of the channels (channel shape and aspect ratio, curvature of the return channels, etc.), the actual temperature differences between the cold and hot fluids, the surface finishing, and the type of materials used.

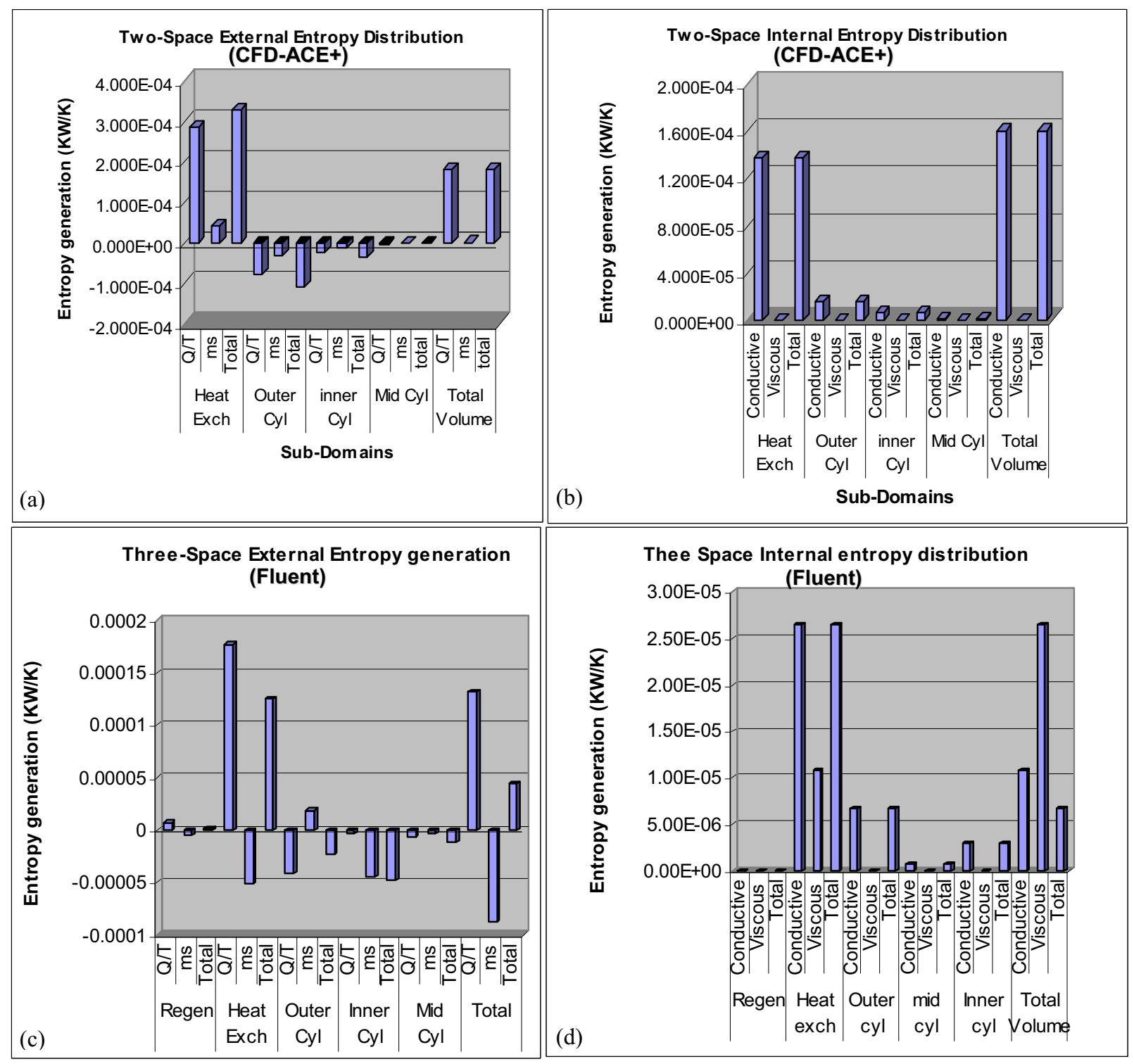

Figures 22. External and Internal Entropy Distribution in the 2-space and 3-space Models

(201.7 RPM, 1.008 MPa., $\mathrm{T}_{\text {wall }}=294 \mathrm{~K}$; Grid size = 147 x 51, \#tspc = 480, at Opt. Cycle =6).

Tables 7 and 8 illustrate entropy generation and availability loss results obtained for the 2-space and 3-space models. For the 2-space model, all CFD-ACE+ results, with the exception of the external entropy generation and external availability energy loss results for the cylinder, are greater than corresponding Sage results. For the 3-space model, all Fluent results, with the exception of the external entropy generation and external availability energy loss results for the heat exchanger are less than corresponding Sage results. Calculation of the percentage differences between internal and external results of entropy and availability energy loss are based on the assumption that external results are more accurate since the calculation of the integral heat transfer rates are more dependable. With Sage, it does not seem to matter what the basis for calculation of the percentage differences is. Calculation of the percentage differences between Sage and the multi-dimensional (multi-D) codes (CFD-ACE+ and Fluent) are based on the 
assumption that results generated by multi-D codes are more accurate since they are more suited to handle multi-D flow situations. Availability energy losses in the heat exchanger are greater than in the cylinder. The least losses are reported in the regenerator, as expected. The numerical errors reflected by the discrepancies between the internal and external entropy generation (or availability energy losses) indicate that Sage does a better job than the multi-D codes in satisfying the entropy generation accounting principle which require that the two methods of calculating for the entropy generation should give the same answer.

Table 7: Entropy Generation and Availability Loss Results (Sage vs. CFD-ACE+)

\begin{tabular}{|c|c|c|c|c|}
\hline \multicolumn{5}{|c|}{ 2-space (Cylinder + Heat Exchanger) } \\
\hline \multirow{3}{*}{ Sub-Domain } & \multicolumn{2}{|c|}{$\begin{array}{c}\text { Sage } \\
\left(\mathrm{T}_{\text {wall }}=294 \mathrm{~K}\right)\end{array}$} & \multicolumn{2}{|c|}{$\begin{array}{c}\text { CFD-ACE+: V2004 (Alpha version) } \\
\left(201.7 \mathrm{RPM}, 1.008 \mathrm{MPa} ., \mathrm{T}_{\text {wall }}=294 \mathrm{~K},\right) \\
(\text { Grid size }=147 \mathrm{X} 51, \# \text { tspc }=480, \text { Opt. Cycle }=6)\end{array}$} \\
\hline & Internal & External & Internal & External \\
\hline & \multicolumn{4}{|c|}{ Entropy Generation $(\mathrm{KW} / \mathrm{K})$} \\
\hline $\begin{array}{c}\text { Heat } \\
\text { Exchanger }\end{array}$ & 0.0000706900 & 0.0000653500 & 0.00013700 & 0.000327000 \\
\hline Cylinder & 0.00002245 & 0.00002198 & 0.000023000 & -0.000146000 \\
\hline Total & 0.0000931400 & 0.00008733 & 0.00016000 & 0.00018100 \\
\hline \multicolumn{5}{|c|}{ Available Energy Loss (KW) } \\
\hline $\begin{array}{c}\text { Heat } \\
\text { Exchanger }\end{array}$ & 0.02120649 & 0.01960549 & 0.0402950 & 0.096142 \\
\hline Cylinder & 0.00673410 & 0.00659400 & 0.006793 & -0.042866 \\
\hline Total & 0.02794059 & 0.02619949 & 0.047088 & 0.053276 \\
\hline AEDiscr & \multicolumn{2}{|c|}{0.00174110} & \multicolumn{2}{|c|}{0.0061880} \\
\hline Sub-Domain & \multicolumn{4}{|c|}{ |\%Difference| between Internal and External Entropy Generation (or AE Loss) } \\
\hline $\begin{array}{c}\text { Heat } \\
\text { Exchanger }\end{array}$ & \multicolumn{2}{|c|}{8.0} & \multicolumn{2}{|c|}{58.0} \\
\hline Cylinder & \multicolumn{2}{|c|}{2.0} & \multicolumn{2}{|c|}{116.0} \\
\hline \multirow[t]{4}{*}{ Total } & \multicolumn{2}{|c|}{7.0} & \multicolumn{2}{|c|}{12.0} \\
\hline & \multicolumn{4}{|c|}{$\mid \%$ Difference $\mid$ between Sage and CFD-ACE+ Results } \\
\hline & \multicolumn{2}{|c|}{ Internal } & \multicolumn{2}{|c|}{ External } \\
\hline & Entropy & AE Loss & Entropy & AE Loss \\
\hline Heat Exch. & 48.4 & 47.4 & 80.0 & 79.6 \\
\hline Cylinder & 2.4 & 0.9 & 115.1 & 115.4 \\
\hline Total & 41.8 & 40.7 & 51.8 & 50.8 \\
\hline
\end{tabular}


Table 8: Entropy Generation and Availability Loss Results (Sage vs. Fluent)

\begin{tabular}{|c|c|c|c|c|}
\hline \multicolumn{5}{|c|}{ 3-space (Cylinder + Heat Exch. + Regenerator) } \\
\hline \multirow{3}{*}{ Sub-Domain } & \multicolumn{2}{|c|}{$\begin{array}{c}\text { Sage } \\
\left(\mathrm{T}_{\text {wall }}=294 \mathrm{~K}\right)\end{array}$} & \multicolumn{2}{|c|}{$\begin{array}{c}\text { Fluent } \\
\left(201.7 \mathrm{RPM}, 1.008 \mathrm{MPa} . \mathrm{T}_{\text {wall }}=294 \mathrm{~K},\right) \\
(\text { Grid size }=147 \mathrm{X} 51, \# \text { tspc }=480, \text { Opt. Cycle }=6)\end{array}$} \\
\hline & Internal & External & Internal & External \\
\hline & \multicolumn{4}{|c|}{ Entropy Generation $(\mathrm{KW} / \mathrm{K})$} \\
\hline Regenerator & 0.0000067568 & 0.0000064611 & 0.0000000192 & 0.0000012722 \\
\hline $\begin{array}{c}\text { Heat } \\
\text { Exchanger }\end{array}$ & 0.0000352724 & 0.0000313046 & 0.00002645 & 0.0001252 \\
\hline Cylinder & 0.00002669 & 0.00002270 & 0.000010281 & -0.00008195 \\
\hline Total & 0.000068718 & 0.00006047 & 0.00003675 & 0.0000445222 \\
\hline \multicolumn{5}{|c|}{ Available Energy Loss (KW) } \\
\hline Regenerator & 0.002251 & 0.00215253 & 0.0000051835 & 0.000343489 \\
\hline $\begin{array}{c}\text { Heat } \\
\text { Exchanger }\end{array}$ & 0.01012557 & 0.00898657 & 0.0067493 & 0.031940386 \\
\hline Cylinder & 0.0076174 & 0.0064784 & 0.00262335 & -0.02020910121 \\
\hline Total & 0.01999397 & 0.0176175 & 0.00937783 & 0.01207477 \\
\hline AEDiscr & \multicolumn{2}{|c|}{0.00237647} & \multicolumn{2}{|c|}{-0.00269694} \\
\hline Sub-Domain & \multicolumn{4}{|c|}{ |\%Difference $\mid$ between Internal and External Entropy Generation (or AE Loss) } \\
\hline Regenerator & \multicolumn{2}{|c|}{4.6} & \multicolumn{2}{|c|}{98.5} \\
\hline $\begin{array}{c}\text { Heat } \\
\text { Exchanger }\end{array}$ & \multicolumn{2}{|c|}{12.7} & \multicolumn{2}{|c|}{78.9} \\
\hline Cylinder & \multicolumn{2}{|c|}{17.6} & \multicolumn{2}{|c|}{113} \\
\hline \multirow[t]{4}{*}{ Total } & \multicolumn{2}{|c|}{13.5} & \multicolumn{2}{|c|}{22.3} \\
\hline & \multicolumn{4}{|c|}{$\mid \%$ Difference $\mid$ between Sage and Fluent Results } \\
\hline & \multicolumn{2}{|c|}{ Internal } & \multicolumn{2}{|c|}{ External } \\
\hline & Entropy & AE Loss & Entropy & AE Loss \\
\hline Regenerator & 99.7 & 99.7 & 80.3 & 84.0 \\
\hline Heat Exch. & 25.0 & 33.3 & 75.0 & 78.8 \\
\hline Cylinder & 61.5 & 65.5 & 27.6 & 67.1 \\
\hline Total & 46.5 & 89.4 & 24.7 & 35.4 \\
\hline
\end{tabular}

The errors for internal entropy and AE loss calculations between Sage and the multi-D codes are noted in general to be smaller than the errors for the corresponding external calculations. The exceptions are the errors reported in the regenerator. The great disparities in the percentage differences between Sage and the multi-D codes results of entropy and AE loss may possibly be due to the inability of the Sage 1-D code to accurately account for temperature and velocity gradients caused by flow separations from walls where there are changes in flow area as for example, between the cylinder and the heat exchanger in the 2-space and 3-space models. Sage 1-D flow is assumed to immediately adjust to the wall boundaries through all geometrical changes in area and can only approximately account for the effect of flow separations, vortex formation, etc. where there are changes in flow area. 


\section{Conclusions and Suggestions for Future Work}

The following have been provided: an overview of the Stirling engine, a discussion of the computer models of the modified MIT 2-space test rig leading to the 3-space model which includes a regenerator, the effect of the regenerator via comparison of the Sage 2-space and 3-space modeling, theoretical development of the thermodynamic loss models, discussion of the numerical simulation results and post-processing of the numerical simulation results to obtain entropy generation/availability energy loss results for the 2-space and 3-space models.

The following conclusions can be drawn from the results of this study which sought to evaluate the effect of adding a regenerator to the MIT 2-space test rig and to characterize the irreversibilities related to heat transfer, mass flow and viscous friction occurring in the modified MIT test rig via entropy generation:

(1) The inclusion of the regenerator:

- reduces the minimum and maximum pressure and work-input values in the cylinder with the exception of the increase in Sage maximum pressure;

- decreases the maximum temperature recorded in the heat exchanger of the 2-space model by $11 \mathrm{~K}$, increases the minimum temperature by $\sim 2 \mathrm{~K}$ and shifts the maximum and minimum temperature values from near the end of the heat exchanger to near the heat exchanger entrance during a cycle;

- leads, on the average, to $\sim 22 \%$ reduction in the heat pumping action in the heat exchanger and $\sim 31 \%$ reduction in the net cycle heat loss (or input work) and whereas Fluent reports $\sim 28 \%$ reduction in the cooling action in the cylinder, Sage reports $\sim 23 \%$ increase in same (both codes report increase in COP);

- reduces the error between the 1-D (Sage) and 2-D (Fluent) code predictions;

- elevates the cylinder space temperatures at stationary points especially at points close to the midpoint of the cylinder clearance volume $(\mathrm{x}=0.001374 \mathrm{~m})$ and piston top center position $(\mathrm{x}=0.002747 \mathrm{~m})$;

- elevates the maximum values of the heat flux and temperature difference at the entrance of the heat exchanger; and

- improves the pressure profile correspondence with Sage result in both the heat exchanger and cylinder spaces and reduces the 2-D peak pressure values in both the cylinder and heat exchanger domains.

(2) The heat exchanger provides the major sub-domain contribution to entropy generation. Thus the heat exchanger heat transfer has the most impact on the 3-space model efficiency followed by outer and inner cylinder heat transfer. Viscous dissipation throughout the entire 3-space domain and mid-cylinder mass transfer and conductive heat transfer contribute minimally to the model's efficiency.

(3) In both the 2-space and 3-space models the two methods of accounting for the entropy generation (external and internal) appear insensitive to the number of cycles of piston motion beyond the third cycle.

(4) The multi-D codes do not satisfy the $2^{\text {nd }}$. Law accounting principle as well as Sage. These may be due to numerical losses.

Clearly, there is room for further studies to extend the boundaries of this study and explore and improve upon the difficulties encountered in this study. For example, other regenerator parameter optimization studies such as wire diameter, annulus diameter (inner and outer) and porosity should be explored with the objective being the maximization of the cylinder-cooling effect. The difficulties encountered incorporating the loss models derived in Section IV in the Fluent code should also be revisited for possible resolution in order to provide a thermal nonequilibrium regenerator modeling capability in addition to the dual zone model provided by the Fluent code.

Typically neglected and often viewed as superfluous, the second law of thermodynamics remains an esoteric and mysterious subject ${ }^{15}$ particularly in computational analysis of thermo-fluid systems. When properly applied, the second law of thermodynamics has proven to be a very powerful tool in the optimization of complex thermodynamic systems. Loss analysis using entropy-generation rates due to heat and fluid flow is a relatively new technique for assessing component performance. It offers a deep insight into the flow phenomena, allows a more exact calculation of losses than is possible with traditional means involving the application of loss correlations and provides an effective tool for improving performance. Designers will know the cumulative amount of all losses computed locally in the flow domain. Entropy generation maps can be produced, and designers can use them by scanning them to detect critical areas (locations in which entropy generation is higher than its integral average value over the entire flow field). By considering the local values of entropy generation rates due to thermal and viscous dissipation, designers can generate a thermodynamically better design by simply trying to avoid these critical areas or recomputing them after a design modification has been introduced to assess local and global effects of the design change. 
Our understanding of loss mechanisms is far from complete. Although numerical predictions are valuable in predicting the heat transfer and flow structure, there are difficulties in predicting the loss accurately. This is due to errors in predicting the boundary layers, transition as well as due to false entropy generation due to numerical dissipation. This work provides a point of reference for incorporation of loss post-processors into Stirling engine numerical codes. The incorporation of a loss post-processor in Stirling engine numerical codes, it is believed, will facilitate the optimization of Stirling engine performance. 


\section{References}

${ }^{1}$ Amiri, A., and Vafai, K., "Transient Analysis of Incompressible Flow through a Packed Bed,” Int. J. Heat Mass Transfer, Vol. 41, pp. 4259-4279, 1998.

${ }^{2}$ Bejan, A., Second-law analysis in heat transfer and thermal design, Adv. Heat Transfer 15 (1982) 1-58.

${ }^{3}$ Bejan, A. 1997. Advanced Engineering Thermodynamics, Second Edition, John Wiley \& Sons, Inc.

${ }^{4}$ Bejan, A., Entropy Generation Minimization: The Method of Thermodynamic Optimization of FiniteTime Systems and Finite-Time Processes, CRC Press, Boca Raton, NY, 1996.

${ }^{5}$ Bejan, A. A study of entropy generation in fundamental convective heat transfer, ASME J. Heat Transfer 101 (1979) 718-725.

${ }^{6}$ Chafe, J.N., “A Study of Gas Spring Heat Transfer in Reciprocating Cryogenic Machinery,” Ph.D. Thesis, Dept. of Mech. Eng., Massachusetts Institute of Technology, Cambridge, MA, 1988.

${ }^{7}$ Cengel, Y. A. and Boles, M. A., Thermodynamics, an Engineering Approach, $5^{\text {th }}$. ed., The McGraw-Hill Companies, Inc., 2006.

${ }^{8}$ Currie, T.C. and Carscallen, W.E. Simulation of trailing edge vortex shedding in a transonic turbine cascade. ASME Paper No. 96-GT-483, 1996.

${ }^{9}$ Denton, J.D., 1993. Loss mechanisms in turbo machines. ASME Paper No. 93-GT-435.

${ }^{10}$ Drost, M. K., Zaworski, J.R., A review of second law analysis techniques applicable to basic thermal science research, ASME, AES 4 (1988) 7-12.

${ }^{11}$ Ebiana, A.B., Savadekar, R.T., Patel, K.V., A., "Entropy Generation/Availability Energy Loss Analysis Inside MIT Gas Spring and '2-space' Test Rigs," Proceedings of $3^{\text {rd }}$. International Energy Conversion Engineering Conference. Aug 15-Aug 18, 2005, San Francisco, California.

${ }^{12}$ Ebiana, A.B., Savadekar, R.T, Vallury, A., " $2^{\text {nd }}$ Law Analysis of Sage and CFD-ACE+ Models of MIT Gas Spring and 2-space Test Rigs", Proceedings of $2^{\text {nd }}$. International Energy Conversion Engineering Conference. Aug 16-Aug 19, 2004, Providence, Rhode Island.

${ }^{13}$ Faulkner, H.B., “An Investigation of Instantaneous Heat Transfer During Compression and Expansion in Reciprocating Gas Handling Equipment," Ph.D. Thesis, Dept. of Mech. Eng., Massachusetts Institute of Technology, Cambridge, MA, 1983.

${ }^{14}$ Gedeon, D., 1999. Sage Pulse Tube Model-Class Reference Guide, Gedeon Associates.

${ }^{15}$ Grad, H., "The Many Faces of Entropy". Communications on Pure and Applied Mathematics, vol. XIV, pp. 323- 354, 1961.

${ }^{16}$ H. Reader, G. T, and Hooper, C., 1983, "Stirling Engines", University Press, Cambridge.

${ }^{17}$ Hamaguchi, K., Hiratsuka, Y. and Miyabe, H., "Effects of Regenerator Size Change on the Stirling Engine Performance", Proceedings of the $26^{\text {th }}$ IECEC, (1991).

${ }^{18}$ Harvey, J. P., "Oscillatory Compressible Flow and Heat Transfer in Porous Media - Application to Cryocooler Regenerators”, Georgia Institute of Technology Ph.D., 2003.

${ }^{19}$ Hofman, K.A., Chiang, S.T., Computational Fluid Dynamics for Engineers Volume II, Engineering Education System, 1993, pp. 15.

${ }^{20}$ Kornhauser, Alan A. “Gas-Wall Heat Transfer During Compression and Expansion”, Ph.D. Thesis, Dept. of Mech. Eng., Massachusetts Institute of Technology, Cambridge, MA, 1989.

${ }^{21}$ Lee, K.P. and Smith, J.L. Jr., "Influence of Cyclic Wall-to-Gas Heat Transfer in Cylinder of the Valved Hot-Gas Engine", Proceedings of the $13^{\text {th }}$. Intersociety Energy Conversion Engineering Conference, Paper No 789195, 1978.

${ }^{22}$ Minkowcyz, W.J., Haji-Sheikh, A. and Vafai, K., “On Departure from Local Thermal Equilibrium in Porous Media due to a Rapidly Changing Heat Source: The Sparrow Number,” Int. J. Heat Mass Transfer, Vol. 42, pp. 3373- 3385, 1999.

${ }^{23}$ Mralidhar, K. and Suzuki, K., "Analysis of Flow and Heat Transfer in a Regenerator Mesh using a NonDarcy Thermally Non-Equilibrium Model,” Int. J. Heat Mass Transfer, Vol. 44, pp. 2493-2504, 2001.

${ }^{24}$ Narusawa, U., The second-law analysis of mixed convection in rectangular ducts, Heat Mass Transfer 37 (2001) 197-203. 
${ }^{25}$ Paoletti, S., Rispoli, F., Sciubba, E., Calculation of exergetic losses in compact heat exchanger passages, ASME AES 10 (1989) 21-29.

${ }^{26}$ R. Gordon Campbell, Foundations of Fluid Flow Theory, Addison-Wesley Publishing Company, Inc., Reading, Massachusetts, 1973.

${ }^{27}$ Recktendwald, G. W., “A Study of Heat Transfer between the Walls and Gas Inside the Cylinder of a Reciprocating Compressor,” University of Minnesota Ph.D. Thesis, 1989.

${ }^{28}$ Sahin, A. Z., Second law analysis of laminar viscous flow through a duct subjected to constant wall temperatures, J. Heat Transfer 120 (1980) 76-83.

${ }^{29}$ Simon, T., "Contributions to Regenerator Modeling," Presentation for Regenerator Modeling Panel Discussion, $2^{\text {nd }}$ International Energy Conversion Engineering Conference, Providence, RI, August 18, 2004.

${ }^{30}$ Singh, C., Tathgir, R.G., and Muralidhar, K., "Comparison of 1-equation and 2-equation Models for Convective Heat Transfer in Saturated Porous Media, "The Institution of Engineers (India) Journal, Vol. 84, MC 3, pp. 104-113, October 2003.

${ }^{31}$ Tannehill, J.C., Anderson, D.A. and Pletcher, R.H., Computational Fluid Mechanics and Heat Transfer, Taylor \& Francis, $2^{\text {nd. }}$ ed., Hemisphere Publishing Corporation, 1984.

${ }^{32}$ Tew, R., Ibrahim, M. and Danila, D., Simon, T. Mantell, S. and Sun L., Gedeon, D., Kelly, K. and McLean J., Wood, G., and Qui, S., "A Microfabricated Involute-Foil Regenerator for Stirling Engines", MASATM-2007-214973 and AIAA-2007-4739.

${ }^{33}$ Tew, R., Simon, T., Gedeon, D., Ibrahim, M. and Rong, W. “An Initial Non-Equilibrium Porous-Media Mo-del for CFD Simulation of Stirling Regenerators", Prepared for $4^{\text {th }}$. International Energy Conversion Engineering Conference, San Diego, CA, June 26-29, 2006.

${ }^{34}$ Tew, R.C., et al, "Overview 2004 of NASA-Stirling Convertor CFD Model Development and Regenerator R\&D Efforts", in proceedings of Space Technology and Applications International Forum (STAIF 2005), Albuquerque, NM February 2005.

${ }^{35}$ Tew, R., "Overview of Stirling Regenerators (Porous Media) CFD Modeling Problems," Presentation for Workshop on Convective Heat Transfer in Porous Media, Hosted by NASA Glenn Research Center, North Olmsted, OH, April 28- 29, 2004.

${ }^{36}$ Tew, R.C. Jr., "Two-Dimensional Compressible Non-Acoustic Modeling of Stirling Machine Type Components" Doctor of Engineering Thesis, Dept. of Mech. Eng., Cleveland State University, Cleveland, OH, 2000.

${ }^{37}$ Thieme, L.G. and Schreiber, J.G., NASA GRC Stirling Technology Overview, " Proceedings of the Space Technology Applications international Forum (STAIF-2003), Albuquerque, NM, edited by M.S. El-Genk, American Institute of Physics, Melville, NY, 2003, AIP CP-654, pp. 613-620, also as NASA/TM--2003-212454, 2003.

${ }^{38}$ Whitaker, S. 1999, The method of volume averaging, Kulwer Academic Publishers, Netherlands. 


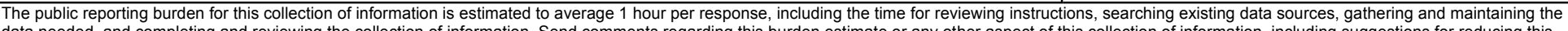

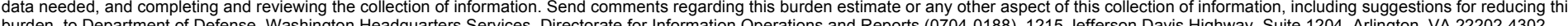

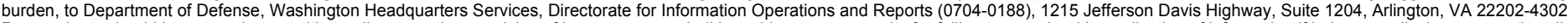

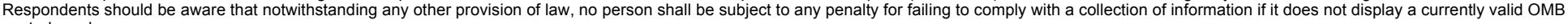
control number.

PLEASE DO NOT RETURN YOUR FORM TO THE ABOVE ADDRESS.
1. REPORT DATE (DD-MM-YYYY)
2. REPORT TYPE

\section{DATES COVERED (From - To)}

01-11-2008

Final Contractor Report

\section{TITLE AND SUBTITLE}

Effect of Adding a Regenerator to Kornhauser's MIT "Two-Space" (Gas-Spring+Heat

Exchanger) Test Rig

5a. CONTRACT NUMBER

5b. GRANT NUMBER

NNC-05AA24A

5c. PROGRAM ELEMENT NUMBER

5d. PROJECT NUMBER

Ebiana, Asuquo, B.; Gidugu, Praveen

5e. TASK NUMBER

5f. WORK UNIT NUMBER

WBS 138494.04.01.01

8. PERFORMING ORGANIZATION

REPORT NUMBER

E-16657

Cleveland State University

2121 Euclid Ave

Cleveland, Ohio 44115

\section{SPONSORING/MONITORING AGENCY NAME(S) AND ADDRESS(ES)}

National Aeronautics and Space Administration

Washington, DC 20546-0001

\section{SPONSORING/MONITORS ACRONYM(S) \\ NASA; AIAA}

\section{SPONSORING/MONITORING REPORT NUMBER \\ NASA/CR-2008-215480; AIAA-2008- 5718}

\section{DISTRIBUTION/AVAILABILITY STATEMENT}

Unclassified-Unlimited

Subject Category: 20

Available electronically at http://gltrs.grc.nasa.gov

This publication is available from the NASA Center for AeroSpace Information, 301-621-0390

\section{SUPPLEMENTARY NOTES}

\section{ABSTRACT}

This study employed entropy-based second law post-processing analysis to characterize the various thermodynamic losses inside a 3 -space solution domain (gas spring+heat exchanger+regenerator) operating under conditions of oscillating pressure and oscillating flow. The 3space solution domain is adapted from the 2-space solution domain (gas spring+heat exchanger) in Kornhauser's MIT test rig by modifying the heat exchanger space to include a porous regenerator system. A thermal nonequilibrium model which assumes that the regenerator porous matrix and gas average temperatures can differ by several degrees at a given axial location and time during the cycle is employed. An important and primary objective of this study is the development and application of a thermodynamic loss post-processor to characterize the major thermodynamic losses inside the 3-space model. It is anticipated that the experience gained from thermodynamic loss analysis of the simple 3-space model can be extrapolated to more complex systems like the Stirling engine. It is hoped that successful development of loss post-processors will facilitate the improvement of the optimization capability of Stirling engine analysis codes through better understanding of the heat transfer and power losses. It is also anticipated that the incorporation of a successful thermal nonequilibrium model of the regenerator in Stirling engine CFD analysis codes, will improve our ability to accurately model Stirling regenerators relative to current multidimensional thermal-equilibrium porous media models.

\section{SUBJECT TERMS}

Gas-spring+heat exchanger; Stirling engine; Thermal nonequilibrium model

\begin{tabular}{|c|c|c|c|c|}
\hline 16. SECURI & ASSIFICATION & & 17. LIMITATION OF & 18. NUMBER \\
\hline $\begin{array}{l}\text { a. REPORT } \\
U\end{array}$ & $\begin{array}{l}\text { b. ABSTRACT } \\
U\end{array}$ & $\begin{array}{l}\text { c. THIS } \\
\text { PAGE } \\
\text { U }\end{array}$ & UU & $\begin{array}{c}\text { PAGES } \\
55\end{array}$ \\
\hline
\end{tabular}

19a. NAME OF RESPONSIBLE PERSON STI Help Desk (email:help@sti.nasa.gov) 19b. TELEPHONE NUMBER (include area code) $301-621-0390$ 

